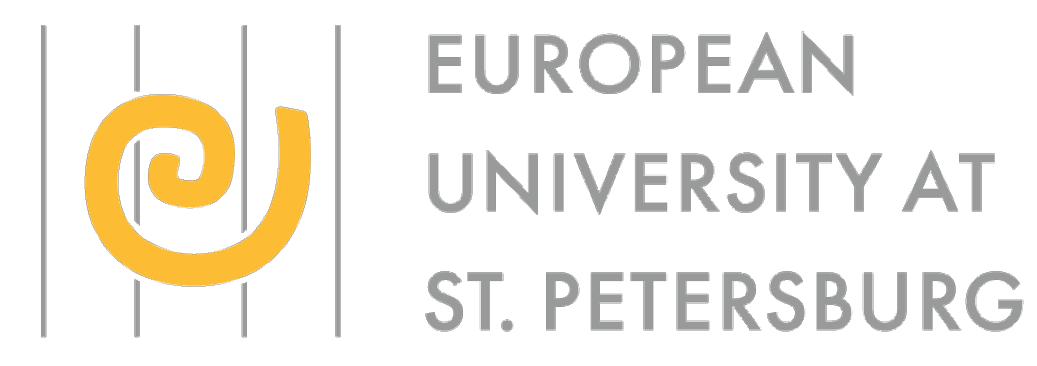

Naive Agents with Quasi-hyperbolic Discounting and Perfect Foresight

by Kirill Borissov, Mikhail Pakhnin,

and Ronald Wendner

$2020 / 03$

Working Paper Series

Department of Economics

European University at St. Petersburg 
Borissov K., Pakhnin M., Wendner R. (2020) Naive Agents with Quasihyperbolic Discounting and Perfect Foresight. European University at St. Petersburg, Department of Economics, Working Paper 2020/03, 48 p.

Abstract: We consider the Ramsey growth model with quasi-hyperbolic discounting where the agent cannot commit to future actions and is naive about her time inconsistency. We study the problem of observational equivalence, i.e., whether consumption paths are the same under quasi-hyperbolic and exponential discounting. To describe the behavior of a naive agent in a general equilibrium framework, we introduce the notion of a sliding equilibrium path and distinguish between two natural types of expectations: pseudo-prefect foresight and perfect foresight. Under pseudo-prefect foresight an agent at each date recalculates both her consumption path and expectations about prices, while under perfect foresight an agent correctly foresees prices on a sliding equilibrium path and is naive only about her time inconsistency. The main contribution of this paper is the study of a sliding equilibrium path under perfect foresight. We prove its existence for a general isoelastic utility function and show that, except for the well-known cases of a constant interest rate or logarithmic utility, there is no observational equivalence in general. In a number of important cases we also compare sliding equilibria under pseudo-perfect and perfect foresight in terms of long-run macroeconomic variables, and show that perfect foresight implies a higher capital stock and a higher consumption level than pseudo-perfect foresight.

Keywords: Quasi-hyperbolic discounting, Observational equivalence, Time inconsistency, Naive agents, Perfect foresight

JEL classification: D15, D91, E21, O40

Kirill Borissov,

European University at St. Petersburg, Russia.

$\bowtie \quad$ kirill@eu.spb.ru

\section{Mikhail Pakhnin,}

European University at St. Petersburg, Russia; CESifo, Munich, Germany.

$\triangle$ mpakhnin@eu.spb.ru

\section{Ronald Wendner,}

University of Graz, Austria.

\ronald.wendner@uni-graz.at

(c) K. Borissov, M. Pakhnin, R. Wendner, 2020 


\title{
Naive Agents with Quasi-hyperbolic Discounting and Perfect Foresight
}

\author{
Kirill Borissov ${ }^{\mathrm{a}, *} \quad$ Mikhail Pakhnin ${ }^{\mathrm{a}, \dagger} \quad$ Ronald Wendner ${ }^{\mathrm{b}, \grave{\dagger}}$ \\ a Department of Economics, European University at St. Petersburg, Russia \\ b Department of Economics, University of Graz, Austria
}

\begin{abstract}
We consider the Ramsey growth model with quasi-hyperbolic discounting where the agent cannot commit to future actions and is naive about her time inconsistency. We study the problem of observational equivalence, i.e., whether consumption paths are the same under quasi-hyperbolic and exponential discounting. To describe the behavior of a naive agent in a general equilibrium framework, we introduce the notion of a sliding equilibrium path and distinguish between two natural types of expectations: pseudo-prefect foresight and perfect foresight. Under pseudo-prefect foresight an agent at each date recalculates both her consumption path and expectations about prices, while under perfect foresight an agent correctly foresees prices on a sliding equilibrium path and is naive only about her time inconsistency. The main contribution of this paper is the study of a sliding equilibrium path under perfect foresight. We prove its existence for a general isoelastic utility function and show that, except for the well-known cases of a constant interest rate or logarithmic utility, there is no observational equivalence in general. In a number of important cases we also compare sliding equilibria under pseudo-perfect and perfect foresight in terms of long-run macroeconomic variables, and show that perfect foresight implies a higher capital stock and a higher consumption level than pseudo-perfect foresight.
\end{abstract}

Keywords: Quasi-hyperbolic discounting; Observational equivalence; Time inconsistency; Naive agents; Perfect foresight

JEL Classification: D15, D91, E21, O40

\footnotetext{
*E-mail: kirill@eu.spb.ru

$\dagger$ †-mail: mpakhnin@eu.spb.ru

$\ddagger$ E-mail: ronald.wendner@uni-graz.at
} 


\section{Introduction}

Standard economic growth models employ the assumption of exponential discounting. While analytically tractable and convenient, this assumption is not supported empirically. A vast amount of laboratory and field studies of time preferences shows that discount rates are higher in the short run than in the long run, i.e., individuals exhibit present bias (see, e.g., Ainslie, 1992; Loewenstein and Prelec, 1992; Frederick et al., 2002; DellaVigna, 2009). A popular and well-known way to model present bias is to assume time-declining (hyperbolic) discounting, as opposed to constant (exponential) discounting (see, e.g., Angeletos et al., 2001, for a discussion and literature overview).

The assumption that individuals are time inconsistent and discount the future hyperbolically does not necessarily imply that the observable outcome of their consumption and savings decisions are different from that of individuals who discount exponentially. In a number of models (see, e.g., Laibson, 1996; Barro, 1999; Krusell et al., 2002), it is shown that the optimal paths of consumption and capital can coincide under hyperbolic and exponential discounting. This phenomenon is discussed in the literature under the header of "observational equivalence".

In this paper we study the question of observational equivalence in the Ramsey growth model with quasi-hyperbolic discounting where the agent cannot commit to future actions and is naive about her time inconsistency. We introduce an equilibrium concept capable of describing the behavior of a naive agent who revises her consumption path at each date, and note that such equilibria critically depend on expectations. We clarify the role of expectations in equilibrium and study the assumptions under which observational equivalence to a standard Ramsey model occurs. Our results show that observational equivalence is not a general phenomenon.

In his pioneering paper on time inconsistency, Strotz (1955) considers a simple growth model and addresses the question of what will happen if an agent reconsiders her optimal path at each date. He shows that the optimal path as viewed from any future date is a truncation of the original optimal path only for an agent with exponential discounting. An agent with another type of discounting (e.g., hyperbolic) is time-inconsistent - at each date she has a new optimal path. Strotz argues that such an agent will learn to distrust her future behavior, and suggests two strategies that restore time consistency. First, the strategy of precommitment where the agent forces herself to follow the original optimal path. Second, the strategy of consistent planning where the agent chooses the best path among those she would actually follow.

Pollak (1968) clarifies the ideas of Strotz and distinguishes between two types of agents who cannot commit to their future actions. First, Pollak introduces a naive agent, who is unaware of her time-inconsistent preferences. A naive agent reconsiders her optimal path at each date, and the outcome of this procedure is referred to as a "naive path". ${ }^{1}$ Second, he introduces a sophisticated agent, who recognizes her time inconsistency. The path obtained under Strotz's strategy of consistent planning is referred to as a "sophisticated path". Pollak proves that in a cake-eating model with logarithmic utility, the naive and sophisticated paths coincide.

\footnotetext{
${ }^{1}$ The notion of a naive path resembles the notion of a sliding path or a rolling plan (see, e.g., Goldman, 1968; Kaganovich, 1985).
} 
Phelps and Pollak (1968) compare naive and sophisticated paths in a model with quasihyperbolic discounting, isoelastic utility and linear production technology (with a constant marginal productivity of capital, i.e., an exogenous and constant interest rate). They note that a sophisticated solution is a Nash equilibrium in a game between different generations, characterize naive and sophisticated paths for general isoelastic utility, and show that, when utility is logarithmic, both paths coincide. ${ }^{2}$

Following Laibson (1997), most of the literature on quasi-hyperbolic discounting is concerned with sophisticated agents. An agent is modeled as a sequence of autonomous temporal selves with conflicting preferences, whose behavior is described by a dynamic game played among the agent's different selves. Sophisticated paths correspond to the equilibria of such a game, which are typically refined to Markov-perfect Nash equilibria. ${ }^{3}$

Many recent studies of the effects of hyperbolic discounting on consumption and savings decisions within a neoclassical growth model employ the assumption of sophisticated agents (see, e.g., Harris and Laibson, 2001; Krusell and Smith, 2003; Maliar and Maliar, 2006; Ekeland and Lazrak, 2010). It is common to analyze sophisticated paths either under logarithmic utility or under the assumption of an exogenous and constant interest rate, primarily because both assumptions facilitate the analysis and allow for analytical solutions. ${ }^{4}$ In particular, Krusell et al. (2002) provide a closed-form solution for the Markov-perfect equilibrium in the Ramsey model with quasi-hyperbolic discounting, logarithmic utility and Cobb-Douglas production technology. Under a constant interest rate, a number of important results are obtained, even for general isoelastic utility (see, e.g., Bernheim et al., 2015; Cao and Werning, 2018).

The literature on growth models with naive agents is much less prolific. In an important contribution, Barro (1999) studies the Ramsey model with time-declining discounting and logarithmic utility. He provides a full solution for an agent who reconsiders her consumption path at each instant of time, i.e., he implicitly assumes a naive agent and obtains a naive path. ${ }^{5}$ Barro also raises the question of observational equivalence, i.e., whether consumption paths are the same under hyperbolic and exponential discounting. He shows observational equivalence for a naive path under logarithmic utility and any concave production function.

The results by Barro (1999) are extended by Findley and Caliendo (2014). They consider the finite-horizon model with quasi-hyperbolic discounting and exogenous interest rate, and prove that for any fixed and constant planning horizon, a naive path is observationally equivalent to the optimal path in a model with exponential discounting in two cases: 1) for logarithmic utility and 2) for general isoelastic utility and a constant interest rate. ${ }^{6}$

The question of which type of agents, naive or sophisticated, is more appropriate in the

\footnotetext{
2 Sophisticated paths in the spirit of Strotz are further studied by Peleg and Yaari (1973) and Goldman (1980).

3 The properties of such equilibria in a very general setting are extensively studied by Sorger (2004).

4 Each of these assumptions allows also to study sophisticated paths under varying levels of commitment possibilities (see Sorger, 2007), as well as for agents who are heterogeneous in their time preferences (see Drugeon and Wigniolle, 2019).

${ }^{5}$ Barro considers a naive agent, but since under logarithmic utility the propensity to consume is constant, his solution looks like a time-consistent one. Perhaps, this is the reason why many subsequent authors mistook Barro's naive agent for a sophisticated agent.

${ }^{6}$ Farzin and Wendner (2014) in a similar model confirm these results and note that hyperbolic discounting and short-term planning imply a hump-shaped dynamics of saving rate which is consistent with empirical evidence.
} 
framework of economic growth theory, is beyond the scope of this paper. There are supporting arguments for both approaches: e.g., naivete is suggested to be closer to the real-world decision making, while sophistication is considered to be more consistent with the standard notion of rationality. In the prior literature, important results about both naive and sophisticated paths are obtained either under logarithmic utility (in which case, roughly speaking, nothing depends on the interest rate), or under an exogenous and constant interest rate. It should be noted that even under these assumptions, a sophisticated path is a complicated game-theoretic notion, and its analysis is technically demanding. ${ }^{7}$ A naive path has a merit in that it allows for a more comprehensive description in terms of general equilibrium theory. In this paper we employ the assumption of naive agents, and obtain results about observational equivalence under fairly general assumptions (i.e., in a general equilibrium framework with an endogenously changing interest rate and isoelastic utility).

It is well known that the standard Ramsey model with exponential discounting exhibits two properties. First, the optimal path of consumption and capital is time-consistent, i.e., re-planning at any $\tau$ does not change the optimal path from $\tau$ onwards, so the optimal path as viewed from date $\tau$ (date- $\tau$ optimal path) is a truncation of the original date- 0 optimal path. Second, the optimal path can be decentralized as an equilibrium path. In equilibrium, an agent who perfectly foresees interest and wage rates chooses the path of consumption and savings (capital) that coincides with the optimal one.

However, in the Ramsey model with quasi-hyperbolic discounting where the agent cannot commit to future actions and is naive about her time inconsistency, both properties generically fail to hold. First, an optimal path is time-inconsistent, i.e., a date- $\tau$ optimal path differs from the truncation of a date-0 optimal path. Second, the traditional "general equilibrium logic" that says that optimal and equilibrium paths are essentially the same does not apply, because, due to time inconsistency, the notion of "perfect foresight" in equilibrium is unclear.

To address the first issue (time inconsistency of an optimal path), we apply an optimality concept termed sliding optimal path. A sliding optimal path consists of only the date- $\tau$ choices of the date- $\tau$ optimal paths of consumption and capital. For each $\tau=0,1, \ldots$, new date- $\tau$ optimal paths are calculated, and of each of those paths, the sliding optimal path picks only the date- $\tau$ elements. We characterize its properties and study observational equivalence, i.e., whether there is a discount factor for which the optimal path in the standard Ramsey model coincides with a sliding optimal path. We note that, to the best of our knowledge, observational equivalence holds for sliding optimal paths only in two cases: the stationary case and the case with logarithmic utility and Cobb-Douglas production technology.

To address the second issue (the role of expectations in equilibrium), we turn from optimal to equilibrium paths. To deal with time inconsistency of an equilibrium path, we introduce an equilibrium concept termed sliding equilibrium path (cf. Borissov, 2013). Clearly, every date- $\tau$ path of consumption and savings (capital) chosen by an agent depends on expectations about future interest and wage rates, and so does every sliding equilibrium path.

\footnotetext{
7 For instance, much less is known about observational equivalence of sophisticated paths. Under logarithmic utility, observational equivalence holds for linear production technology (as follows from Phelps and Pollak, 1968), and for Cobb-Douglas production technology (see Krusell et al., 2002).
} 
We distinguish between two types of expectations: pseudo-perfect foresight and perfect foresight. Under pseudo-perfect foresight, at each date $\tau$, an agent expects those interest and wage rates that occur on a date- $\tau$ equilibrium path, as if this path would be followed for all future dates $t>\tau$. However, due to naivete, at each $t>\tau$, the agent recalculates her consumption path, and therefore also recalculates expectations of future equilibrium interest and wage rates. A sliding equilibrium path under pseudo-perfect foresight (which picks only the date- $\tau$ elements of each date- $\tau$ equilibrium path) is a decentralization of a sliding optimal path. Hence observational equivalence for sliding equilibrium paths under pseudo-perfect foresight is the same as for sliding optimal paths.

Pseudo-perfect foresight implies that an agent is naive both about her future preferences and future prices. This leads us to consider another reasonable type of expectations, namely, perfect foresight. Under perfect foresight, at each date an agent expects those interest and wage rates that occur on the resulting sliding equilibrium path under perfect foresight. Hence a naive agent with perfect foresight is only partially naive - she remains unaware of her time-inconsistent preferences, but correctly foresees prices. ${ }^{8}$

We prove that for a general isoelastic utility function a sliding equilibrium path under perfect foresight exists, and study the question of observational equivalence, i.e., whether a consumption path on a sliding equilibrium path under perfect foresight coincides with that in a standard Ramsey model. We show that a sliding equilibrium path under perfect foresight is observationally equivalent to an optimal path in the standard Ramsey model in two cases: for logarithmic utility and for a stationary sliding equilibrium under general isoelastic utility. Moreover, we prove that these are the only cases where observational equivalence holds within the class of isoelastic utility functions, there is no observational equivalence beyond the standard cases of logarithmic utility and a constant interest rate.

We also compare sliding equilibria under pseudo-perfect and perfect foresight in terms of long-run macroeconomic variables for the stationary case and the case with logarithmic utility and Cobb-Douglas production technology. Our results suggest that perfect foresight always implies a higher saving rate and a higher long-run consumption level than pseudo-perfect foresight.

The rest of the paper is organized as follows. Section 2 provides simple examples motivating and illustrating the subsequent analysis. In Section 3 we consider a sliding optimal path and its decentralization - a sliding equilibrium path under pseudo-perfect foresight. Section 4 introduces the central object of our study, a sliding equilibrium path under perfect foresight, and presents our main results. Section 5 concludes. The Appendix contains the proofs and mathematical details that support the analysis in the main text.

\footnotetext{
${ }^{8}$ Following O'Donoghue and Rabin (2001), the literature on partial naivete typically assumes that a partially naive agent recognizes her present bias, but underestimates its impact. Our paper contributes to the discussion of partial naivete by noting that in a general equilibrium framework an agent can have perfect foresight about prices, but can be naive about her time inconsistency.
} 


\section{Motivational examples and basic facts}

\subsection{Motivational examples}

Consider an infinitely lived agent with quasi-hyperbolic $(\beta-\delta)$ discounting. Her (intertemporal) utility at each date $\tau$ is given by

$$
u\left(c_{\tau}\right)+\beta \sum_{t=\tau+1}^{\infty} \delta^{t-\tau} u\left(c_{t}\right)=u\left(c_{\tau}\right)+\beta \delta u\left(c_{\tau+1}\right)+\beta \delta^{2} u\left(c_{\tau+2}\right)+\ldots,
$$

where $0<\beta<1$ is the present bias parameter, $0<\delta<1$ is the long-run discount factor, and $u(c)$ is the isoelastic instantaneous utility function:

$$
u(c)=\frac{c^{1-\rho}}{1-\rho}, \quad \rho>0,
$$

with the convention that $\rho=1$ refers to the $\log$ arithmic case $u(c)=\ln c$. The agent cannot commit to her future actions and is naive, i.e., unaware of her time-inconsistent preferences.

Suppose at date $\tau$ the agent maximizes her utility under the intertemporal budget constraint

$$
c_{\tau}+\frac{c_{\tau+1}}{1+r}+\ldots+\frac{c_{\tau+t}}{(1+r)^{t}}+\ldots \leq(1+r) s_{\tau-1}^{*}+w+\frac{w}{1+r}+\ldots+\frac{w}{(1+r)^{t}}+\ldots,
$$

where the initial savings $s_{\tau-1}^{*}$ and constant interest and wage rates, $r$ and $w$, are taken as given by the agent. ${ }^{9}$

Substituting the first-order conditions for this utility maximization problem,

$$
c_{\tau+1}=(\beta \delta(1+r))^{\frac{1}{\rho}} c_{\tau}, \quad c_{t+1}=(\delta(1+r))^{\frac{1}{\rho}} c_{t}, \quad t=\tau+1, \tau+2, \ldots,
$$

into the budget constraint (which holds as equality), we obtain that for the agent with quasihyperbolic discounting the optimal date- $\tau$ consumption in the date- $\tau$ problem, $c_{\tau}^{*}$, is a constant fraction of her expected total wealth:

$$
\begin{aligned}
c_{\tau}^{*} & =\frac{(1+r) s_{\tau-1}^{*}+w+\frac{w}{1+r}+\ldots+\frac{w}{(1+r)^{t}}+\ldots}{1+(\beta \delta)^{\frac{1}{\rho}}(1+r)^{\frac{1-\rho}{\rho}}+\ldots+\left(\beta \delta^{t+1}\right)^{\frac{1}{\rho}}\left((1+r)^{t+1}\right)^{\frac{1-\rho}{\rho}}+\ldots} \\
& =\frac{1-\delta^{\frac{1}{\rho}}(1+r)^{\frac{1-\rho}{\rho}}}{1-\delta^{\frac{1}{\rho}}(1+r)^{\frac{1-\rho}{\rho}}+(\beta \delta)^{\frac{1}{\rho}}(1+r)^{\frac{1-\rho}{\rho}}}\left((1+r) s_{\tau-1}^{*}+w+\frac{w}{1+r}+\ldots+\frac{w}{(1+r)^{t}}+\ldots\right) .
\end{aligned}
$$

Respectively, the optimal date- $\tau$ savings are given by

$$
s_{\tau}^{*}=(1+r) s_{\tau-1}^{*}+w-c_{\tau}^{*} .
$$

Note that the propensities to consume and save out of the expected total wealth depend on the interest rate $r$, but do not depend on savings $s_{\tau-1}^{*}$ and the wage rate $w$. Thus, given the initial

\footnotetext{
9 This example is a reformulation of a model considered by Phelps and Pollak (1968).
} 
savings at date $t=-1, s_{-1}^{*}$, we can recursively construct the sequence $\left\{c_{\tau}^{*}, s_{\tau}^{*}\right\}_{\tau=0}^{\infty}$, which is by construction a naive path, i.e., a solution to the utility maximization problem for the agent with $\beta-\delta$ discounting who reconsiders her consumption path at each date.

Let us compare the above naive path with the solution to the similar problem for an agent with exponential discounting:

$$
\begin{array}{ll}
\max _{\left\{c_{t}\right\}} & u\left(c_{0}\right)+\gamma u\left(c_{1}\right)+\gamma^{2} u\left(c_{2}\right)+\ldots, \\
\text { s. t. } & c_{0}+\frac{c_{1}}{1+r}+\ldots+\frac{c_{t}}{(1+r)^{t}}+\ldots \leq(1+r) s_{-1}^{*}+w+\frac{w}{1+r}+\ldots+\frac{w}{(1+r)^{t}}+\ldots \\
& c_{t} \geq 0, \quad t=0,1, \ldots,
\end{array}
$$

where $0<\gamma<1$ is a constant discount factor, and the initial savings $s_{-1}^{*}$ are the same as above. Repeating the same argument, it is easily seen that the solution to the utility maximization problem for the agent with $\gamma$ discounting, $\left\{c_{\tau}^{* *}\right\}_{\tau=0}^{\infty}$, is recursively constructed by

$$
c_{\tau}^{* *}=\left(1-\gamma^{\frac{1}{\rho}}(1+r)^{\frac{1-\rho}{\rho}}\right)\left((1+r) s_{\tau-1}^{* *}+w+\frac{w}{1+r}+\ldots+\frac{w}{(1+r)^{t}}+\ldots\right)
$$

where the sequence of savings $\left\{s_{\tau}^{* *}\right\}_{\tau=0}^{\infty}$ is given by

$$
s_{\tau}^{* *}=(1+r) s_{\tau-1}^{* *}+w-c_{\tau}^{* *} .
$$

Hence for the agent with exponential discounting the optimal date- $\tau$ consumption for any $\tau$ is also a constant fraction of her expected total wealth.

Now consider the following question: given $\beta$ and $\delta$, can we find $\gamma$ such that $\left\{c_{\tau}^{*}, s_{\tau}^{*}\right\}_{\tau=0}^{\infty}$ coincides with $\left\{c_{\tau}^{* *}, s_{\tau}^{* *}\right\}_{\tau=0}^{\infty}$ ? This question is addressed in the previous literature under the header of "observational equivalence" (see Barro, 1999, for a pioneering contribution). It is easily seen that in this example the answer is affirmative: when $\gamma$ is chosen such that

$$
\gamma^{\frac{1}{\rho}}=\frac{(\beta \delta)^{\frac{1}{\rho}}}{1-\delta^{\frac{1}{\rho}}(1+r)^{\frac{1-\rho}{\rho}}+(\beta \delta)^{\frac{1}{\rho}}(1+r)^{\frac{1-\rho}{\rho}}},
$$

we have $c_{\tau}^{*}=c_{\tau}^{* *}$ and $s_{\tau}^{*}=s_{\tau}^{* *}$ for all $\tau$, and thus $\left\{c_{\tau}^{*}, s_{\tau}^{*}\right\}_{\tau=0}^{\infty}$ and $\left\{c_{\tau}^{* *}, s_{\tau}^{* *}\right\}_{\tau=0}^{\infty}$ precisely coincide.

To some extent the above argument can be generalized to the case where the interest and wage rates change over time. Suppose that the utility function is logarithmic, and the agent with quasi-hyperbolic discounting solves the following problem at date $\tau$ :

$$
\begin{array}{ll}
\max _{\left\{c_{t}\right\}} & \ln c_{\tau}+\beta \sum_{t=\tau+1}^{\infty} \delta^{t-\tau} \ln c_{t} \\
\text { s. t. } & c_{\tau}+\frac{c_{\tau+1}}{1+r_{\tau+1}}+\ldots+\frac{c_{\tau+t}}{\left(1+r_{\tau+1}\right) \cdots\left(1+r_{\tau+t}\right)}+\ldots \\
& \leq\left(1+r_{\tau}\right) s_{\tau-1}+w_{\tau}+\frac{w_{\tau+1}}{1+r_{\tau+1}}+\ldots+\frac{w_{\tau+t}}{\left(1+r_{\tau+1}\right) \cdots\left(1+r_{\tau+t}\right)}+\ldots \\
& c_{t} \geq 0, \quad t=\tau, \tau+1, \ldots
\end{array}
$$


where the initial savings $s_{\tau-1}^{*}$ and the sequences of interest and wage rates, $\left\{r_{t}\right\}_{t=\tau}^{\infty}$ and $\left\{w_{t}\right\}_{t=\tau}^{\infty}$, are taken as given by the agent. ${ }^{10}$

Again, substituting the first-order conditions

$$
c_{\tau+1}=\beta \delta\left(1+r_{\tau+1}\right) c_{\tau}, \quad c_{t+1}=\delta\left(1+r_{t+1}\right) c_{t}, \quad t=\tau+1, \tau+2, \ldots
$$

into the budget constraint, which holds as equality, we obtain that for all $\tau$, the optimal date- $\tau$ consumption in the date- $\tau$ problem, $c_{\tau}^{*}$, for the agent with quasi-hyperbolic discounting is given by a constant fraction of expected total wealth:

$$
\begin{aligned}
c_{\tau}^{*} & =\frac{\left(1+r_{\tau}\right) s_{\tau-1}^{*}+w_{\tau}+\frac{w_{\tau+1}}{1+r_{\tau+1}}+\ldots+\frac{w_{\tau+t}}{\left(1+r_{\tau+1}\right) \cdots\left(1+r_{\tau+t}\right)}+\ldots}{1+\beta \delta+\ldots+\beta \delta^{t+1}+\ldots} \\
& =\frac{1-\delta}{1-\delta+\beta \delta}\left(\left(1+r_{\tau}\right) s_{\tau-1}^{*}+w_{\tau}+\frac{w_{\tau+1}}{1+r_{\tau+1}}+\ldots+\frac{w_{\tau+t}}{\left(1+r_{\tau+1}\right) \cdots\left(1+r_{\tau+t}\right)}+\ldots\right) .
\end{aligned}
$$

Similarly, for the agent with exponential discounting who has a constant discount factor $\gamma$ and logarihmic utility, the optimal date- $\tau$ consumption, $c_{\tau}^{* *}$, provided that initial savings are $s_{\tau-1}^{*}$, is given by

$$
c_{\tau}^{* *}=(1-\gamma)\left(\left(1+r_{\tau}\right) s_{\tau-1}^{*}+w_{\tau}+\frac{w_{\tau+1}}{1+r_{\tau+1}}+\ldots+\frac{w_{\tau+t}}{\left(1+r_{\tau+1}\right) \cdots\left(1+r_{\tau+t}\right)}+\ldots\right)
$$

It is easily seen that $c_{\tau}^{*}=c_{\tau}^{* *}$ for all $\tau$, if and only if

$$
\gamma=\frac{\beta \delta}{1-\delta+\beta \delta}
$$

Moreover, since a naive agent with $\beta-\delta$ discounting constantly recalculates her consumption plan, the result of these recalculations exactly coincides with the optimal consumption plan for the agent with $\gamma$ discounting. Thus, there is again observational equivalence.

In the above two simple examples, we obtain observational equivalence from the perspective of a naive agent who takes the interest and wage rates as given. It is natural to conjecture that these two examples can be extended to a more general framework that includes both the production and consumption sides of the economy, because the interest rate is determined by the marginal product of capital and the wage rate is equal to the marginal product of labor. This argument suggests that observational equivalence is a fairly general phenomenon.

In what follows we clarify the ideas about observational equivalence in a general equilibrium framework. We introduce sliding optimal paths and distinguish between two types of sliding equilibrium paths (SEPs): a SEP under pseudo-perfect foresight, which is a decentralization of a sliding optimal path, and a SEP under perfect foresight, which is a novel object. We study their properties and prove that, within the class of isoelastic utility functions, observational equivalence for a SEP under perfect foresight does not hold, except for the considered above cases of a constant interest rate and logarithmic utility.

10 This example resembles the model considered by Barro (1999). 


\subsection{Basic facts}

To proceed with our analysis, it is necessary to recall the well-known facts and results concerning the discrete-time Ramsey model that will be useful in what follows.

Consider the standard Ramsey model, i.e., the optimal growth model with exponential discounting (Ramsey, 1928). Given an initial capital stock $k_{0}>0$, the planner solves the following problem at date 0 :

$$
\begin{aligned}
\max _{\left\{c_{t}, k_{t+1}\right\}} & \sum_{t=0}^{\infty} \gamma^{t} u\left(c_{t}\right), \\
\text { s. t. } & c_{t}+k_{t+1}=f\left(k_{t}\right), \\
& c_{t} \geq 0, \quad k_{t+1} \geq 0, \quad t=0,1, \ldots .
\end{aligned}
$$

Here capital depreciates completely within the period, and the production function $f(k)$ satisfies the standard assumptions:

$$
f(0)=0, \quad f^{\prime}(k)>0, \quad f^{\prime \prime}(k)<0, \quad \exists \bar{k}: f(\bar{k})=\bar{k}, \quad \gamma f^{\prime}(0)>1 .
$$

A solution to problem (2), the optimal path in the standard Ramsey model with the discount factor $\gamma$, we will call the $\gamma$-optimal path starting from $k_{0}$. Note that the planner with exponential discounting is time-consistent - the $\gamma$-optimal path chosen at date 0 once and for all remains optimal at any future date.

The optimal path in the standard Ramsey model can be decentralized as an equilibrium path. Consider the general equilibrium version of the standard Ramsey model (Cass, 1965; Koopmans, 1965). At the consumption side, a representative agent solves the following problem at date 0 , given an initial capital stock $s_{-1}=k_{0}$ :

$$
\begin{aligned}
\max _{\left\{c_{t}\right\}} & \sum_{t=0}^{\infty} \gamma^{t} u\left(c_{t}\right) \\
\text { s. t. } & c_{0}+\frac{c_{1}}{1+r_{1}}+\ldots+\frac{c_{t+1}}{\left(1+r_{1}\right) \cdots\left(1+r_{t+1}\right)}+\ldots \\
& \leq\left(1+r_{0}\right) s_{-1}+w_{0}+\frac{w_{1}}{1+r_{1}}+\ldots+\frac{w_{t+1}}{\left(1+r_{1}\right) \cdots\left(1+r_{t+1}\right)}+\ldots, \\
& c_{t} \geq 0, \quad t=0,1, \ldots
\end{aligned}
$$

where interest rates $\left\{r_{t}\right\}_{t=0}^{\infty}$ and wage rates $\left\{w_{t}\right\}_{t=0}^{\infty}$ are taken as given by the agent.

At the production side, a representative firm with production function $f(k)$ at each date $t$ solves the following myopic profit maximization problem:

$$
\begin{aligned}
& \max _{k_{t}} f\left(k_{t}\right)-\left(1+r_{t}\right) k_{t}, \\
& \text { s. t. } k_{t} \geq 0,
\end{aligned}
$$

where the interest rate $r_{t}$ is taken as given by the firm, and the residual profit is treated as the wage rate. 
The equilibrium path in the standard Ramsey model with the discount factor $\gamma$, which we will call a $\gamma$-equilibrium path starting from $s_{-1}$, is a sequence $\left\{\tilde{c}_{t}, \tilde{s}_{t}, \tilde{k}_{t+1}, \tilde{r}_{t}, \tilde{w}_{t}\right\}_{t=0}^{\infty}$, defined naturally by the following conditions. First, the agent maximizes utility correctly foreseeing the prices, i.e., $\left\{\tilde{c}_{t}\right\}_{t=0}^{\infty}$ is the solution to problem (3) at given $\left\{\tilde{r}_{t}\right\}_{t=0}^{\infty}$ and $\left\{\tilde{w}_{t}\right\}_{t=0}^{\infty}$, and savings $\left\{\tilde{s}_{t}\right\}_{t=0}^{\infty}$ are recursively determined by $\tilde{s}_{t}=\left(1+\tilde{r}_{t}\right) \tilde{s}_{t-1}+\tilde{w}_{t}-\tilde{c}_{t}$. Second, the firm maximizes profits, so prices at each date $t$ are equal to the corresponding marginal products: $1+\tilde{r}_{t}=f^{\prime}\left(\tilde{k}_{t}\right)$ and $\tilde{w}_{t}=f\left(\tilde{k}_{t}\right)-f^{\prime}\left(\tilde{k}_{t}\right) \tilde{k}_{t}$. Third, the capital market clears at each date, i.e., savings are equal to the investment: $\tilde{s}_{t-1}=\tilde{k}_{t}$.

It is well known that in the standard Ramsey model equilibrium and optimal paths are essentially the same - the sequence $\left\{\tilde{c}_{t}, \tilde{k}_{t+1}\right\}_{t=0}^{\infty}$ extracted from the $\gamma$-equilibrium path starting from $s_{-1}$ solves problem (2), i.e., coincides with the $\gamma$-optimal path starting from $k_{0}=s_{-1}$.

Note that the solution to problem (3) is a consumer optimum at given interest and wage rates under exponential discounting. We also need the definition of a date- $\tau$ consumer optimum under quasi-hyperbolic discounting. Given initial savings $s_{\tau-1}$, consider the following problem at date $\tau$ :

$$
\begin{aligned}
\max _{\left\{c_{t}\right\}} & u\left(c_{\tau}\right)+\beta \sum_{t=\tau+1}^{\infty} \delta^{t-\tau} u\left(c_{t}\right) \\
\text { s. t. } & c_{\tau}+\frac{c_{\tau+1}}{1+r_{\tau+1}}+\ldots+\frac{c_{\tau+t}}{\left(1+r_{\tau+1}\right) \cdots\left(1+r_{\tau+t}\right)}+\ldots \\
& \leq\left(1+r_{\tau}\right) s_{\tau-1}+w_{\tau}+\frac{w_{\tau+1}}{1+r_{\tau+1}}+\ldots+\frac{w_{\tau+t}}{\left(1+r_{\tau+1}\right) \cdots\left(1+r_{\tau+t}\right)}+\ldots \\
& c_{t} \geq 0, \quad t=\tau, \tau+1, \ldots
\end{aligned}
$$

where $u(c)$ is of the form (1), and interest rates $\left\{r_{t}\right\}_{t=\tau}^{\infty}$ and wage rates $\left\{w_{t}\right\}_{t=\tau}^{\infty}$ are taken as given by the agent.

A sequence $\left\{c_{t}^{\tau}, s_{t}^{\tau}\right\}_{t=\tau}^{\infty}$ is called a date- $\tau$ consumer optimum starting from $s_{\tau-1}$ at given $\left\{r_{t}\right\}_{t=\tau}^{\infty}$ and $\left\{w_{t}\right\}_{t=\tau}^{\infty}$, if $\left\{c_{t}^{\tau}\right\}_{t=\tau}^{\infty}$ is a solution to problem (5) and the corresponding savings $\left\{s_{t}^{\tau}\right\}_{t=\tau}^{\infty}$ are determined recursively by

$$
s_{t}^{\tau}=\left(1+r_{t}^{\tau}\right) s_{t-1}^{\tau}+w_{t}^{\tau}-c_{t}^{\tau}, \quad t=\tau, \tau+1, \ldots
$$

If a solution to problem (5) exists, it satisfies the budget constraint as equality and the following first-order conditions:

$$
c_{\tau+1}^{\tau}=\left(\beta \delta\left(1+r_{\tau+1}\right)\right)^{\frac{1}{\rho}} c_{\tau}^{\tau}, \quad c_{t}^{\tau}=\left(\delta\left(1+r_{t}\right)\right)^{\frac{1}{\rho}} c_{t-1}^{\tau}, \quad t=\tau+2, \tau+3, \ldots
$$

Substituting the first-order conditions into the budget constraint, we obtain the expression for the date- $\tau$ consumption in a date- $\tau$ consumer optimum:

$$
c_{\tau}^{\tau}=\frac{\left(1+r_{\tau}\right) s_{\tau-1}+w_{\tau}+\frac{w_{\tau+1}}{1+r_{\tau+1}}+\ldots+\frac{w_{\tau+t}}{\left(1+r_{\tau+1}\right) \cdots\left(1+r_{\tau+t}\right)}+\ldots}{1+(\beta \delta)^{\frac{1}{\rho}}\left(1+r_{\tau+1}\right)^{\frac{1-\rho}{\rho}}+\ldots+\left(\beta \delta^{t}\right)^{\frac{1}{\rho}}\left(1+r_{\tau+1}\right)^{\frac{1-\rho}{\rho}} \cdots\left(1+r_{\tau+t}\right)^{\frac{1-\rho}{\rho}}+\ldots} .
$$




\section{Sliding optimal paths and their decentralization}

The main contribution of this paper is the study of a sliding equilibrium path under perfect foresight. To understand this notion, it is important to distinguish between three different objects - sliding optimal path (SOP), sliding equilibrium path (SEP) under pseudo-perfect foresight, and SEP under perfect foresight.

To make our definitions more instructive and transparent, we start with the first two objects, since they are simpler to handle. In this section we propose the definition of a SOP, which is a natural concept to describe the behavior of a time-inconsistent planner. Further, we decentralize a SOP and note that on a corresponding equilibrium path an agent at each date revises her expectations about prices, which implies that her foresight is "pseudo-perfect". We introduce a SEP under pseudo-perfect foresight as a decentralized SOP, and characterize its properties.

\subsection{Sliding optimal path}

Our main workhorse is the discrete-time Ramsey model with quasi-hyperbolic discounting. Given an initial capital stock $k_{0}>0$, consider the following date-0 utility maximization problem:

$$
\begin{aligned}
\max _{\left\{c_{t}, k_{t+1}\right\}} & u\left(c_{0}\right)+\beta \sum_{t=1}^{\infty} \delta^{t} u\left(c_{t}\right), \\
\text { s. t. } & c_{t}+k_{t+1}=f\left(k_{t}\right), \\
& c_{t} \geq 0, \quad k_{t+1} \geq 0, \quad t=0,1, \ldots,
\end{aligned}
$$

where $u(c)$ is of the form (1). A solution to problem (7), $\left\{c_{t}^{* 0}, k_{t+1}^{* 0}\right\}_{t=0}^{\infty}$, is the date-0 optimal path (under quasi-hyperbolic discounting) starting from $k_{0}$. As is well known, there exists a unique date-0 optimal path starting from any initial capital stock.

In problem (7), the sum of discounted utilities is maximized once and for all from the date 0 perspective. If the planner is able to commit to her decisions in the future, then she would follow her optimal path chosen at date 0 . However, if she cannot commit to future actions, at each date $\tau$ ("today") she reconsiders the optimal path chosen at date $\tau-1$ ("yesterday"). At any date $\tau$, given capital stock $k_{\tau}$, the planner solves a new problem:

$$
\begin{aligned}
\max _{\left\{c_{t}, k_{t+1}\right\}} & u\left(c_{\tau}\right)+\beta \sum_{t=\tau+1}^{\infty} \delta^{t-\tau} u\left(c_{t}\right), \\
\text { s. t. } & c_{t}+k_{t+1}=f\left(k_{t}\right), \\
& c_{t} \geq 0, \quad k_{t+1} \geq 0, \quad t=\tau, \tau+1, \ldots
\end{aligned}
$$

A solution to problem (8), $\left\{c_{t}^{* \tau}, k_{t+1}^{* \tau}\right\}_{t=\tau}^{\infty}$, is the date- $\tau$ optimal path (under quasi-hyperbolic discounting) starting from $k_{\tau}$. Under the standard assumptions, for any $\tau$ there is a unique date- $\tau$ optimal path starting from any capital stock.

Note that the date- $\tau$ optimal path differs from the truncation of the optimal path at any previous date. Indeed, the discount factor between periods $\tau+1$ and $\tau$ equals $\beta \delta$ from the 
date $\tau$ perspective, while it is equal to $\delta$ from any earlier perspective. Therefore, the value $c_{t}^{* \tau}$ planned at date $\tau$ for the date- $t$ consumption will not be optimal when date $t$ comes, which implies that optimal paths under quasi-hyperbolic discounting are time-inconsistent.

We assume that the planner is naive, i.e., she does not recognize her time inconsistency. A natural way to describe the behavior of a naive planner is to consider a step-by-step procedure where the planner at each date recalculates her optimal path and implements only the first step of her optimal plan. We call the outcome of this procedure a sliding optimal path (SOP). Formally, the following definition applies.

Definition 1. A sequence $\left\{c_{t}^{\circ}, k_{t+1}^{\circ}\right\}_{t=0}^{\infty}$ is a sliding optimal path starting from $k_{0}$ in the Ramsey model with quasi-hyperbolic discounting, if for each $\tau \geq 0$, the consumption level and capital stock at date $\tau$ are obtained from the date- $\tau$ optimal path under quasi-hyperbolic discounting starting from $k_{\tau}^{\circ}$ :

$$
c_{\tau}^{\circ}=c_{\tau}^{* \tau}, \quad k_{\tau+1}^{\circ}=k_{\tau+1}^{* \tau} .
$$

Clearly, a SOP exists and is unique. Note that a SOP is essentially characterized by the first step in problem (8), i.e., by the first elements from the date- $\tau$ optimal path $\left\{c_{t}^{* \tau}, k_{t+1}^{* \tau}\right\}_{t=\tau}^{\infty}$. After the first step is implemented at date $\tau\left(c_{\tau}^{* \tau}\right.$ is consumed and $k_{\tau+1}^{* \tau}$ remains as the new capital stock), the planner in fact solves problem (2) with the constant discount factor $\delta$. Therefore, the truncation of the date- $\tau$ optimal path which starts at date $\tau+1,\left\{c_{t}^{* \tau}, k_{t+1}^{* \tau}\right\}_{t=\tau+1}^{\infty}$, is the $\delta$-optimal path starting from $k_{\tau+1}^{* \tau}$. It follows that $c_{t}^{* \tau}$ and $k_{t+1}^{* \tau}$ converge to the corresponding modified golden rule consumption level and capital stock for the discount factor $\delta$. In particular, if the planner can commit to her date- $\tau$ optimal path, in the long run she would enjoy the same consumption as on the $\delta$-optimal path.

This observation allows us to describe the first step in problem (8) and therefore a SOP in terms of dynamic programming (see Appendix A). The interesting question to ask is whether a SOP under quasi-hyperbolic discounting coincides with some $\gamma$-optimal path. We formally define observational equivalence of optimal paths as follows.

Definition 2. A sliding optimal path in the Ramsey model with quasi-hyperbolic discounting, $\left\{c_{t}^{\circ}, k_{t+1}^{\circ}\right\}_{t=0}^{\infty}$, is observationally equivalent to the $\gamma$-optimal path if there exists $\gamma$ for which $\left\{c_{t}^{\circ}, k_{t+1}^{\circ}\right\}_{t=0}^{\infty}$ is a solution to problem (2).

To the best of our knowledge, a SOP under quasi-hyperbolic discounting is observationally equivalent to a $\gamma$-optimal path only in the following two cases. First, in the case of a stationary sliding optimum (SSO).

Definition 3. A pair $\left\{c^{\circ}, k^{\circ}\right\}$ is a stationary sliding optimum if the sequence $\left\{c_{t}^{\circ}, k_{t+1}^{\circ}\right\}_{t=0}^{\infty}$, where for each $t=0,1, \ldots, c_{t}^{\circ}=c^{\circ}$ and $k_{t+1}^{\circ}=k^{\circ}$, is a sliding optimal path starting from $k^{\circ}$.

It is clear that an SSO is observationally equivalent to a stationary $\gamma^{\circ}$-optimum for $\gamma^{\circ}=$ $1 / f^{\prime}\left(k^{\circ}\right)$. It can be checked that $\gamma^{\circ}<\delta$ (see Appendix A).

Second, in the case of logarithmic utility and Cobb-Douglas production technology, as the following claim shows. 
Claim 1. Suppose that $u(c)=\ln c$ and $f(k)=k^{\alpha}$. Then a sliding optimal path in the Ramsey model with quasi-hyperbolic discounting is observationally equivalent to the $\gamma^{\circ}$-optimal path, where $\gamma^{\circ}=\frac{\beta \delta}{1-\alpha \delta+\alpha \beta \delta}$.

Proof. See Appendix A. ${ }^{11}$

Claim 1 implies that in the simple case of logarithmic utility and Cobb-Douglas production technology, observing only the path $\left\{c_{t}^{\circ}, k_{t+1}^{\circ}\right\}_{t=0}^{\infty}$, one cannot determine whether the planner has $\beta-\delta$ discounting, is time-inconsistent and naive; or the planner has $\gamma^{\circ}$ discounting and is time-consistent. Note that the equivalent discount factor $\gamma^{\circ}$ lies in between the short-run discount factor $\beta \delta$ and the long-run discount factor $\delta\left(\beta \delta<\gamma^{\circ}<\delta\right)$, and essentially depends on the technology parameter $\alpha$.

\subsection{Sliding equilibrium path under pseudo-perfect foresight}

The natural question is whether a SOP can be decentralized as an equilibrium path. Recall that a SOP is constructed by applying at each date $\tau$ the corresponding date- $\tau$ optimal path. Therefore, to decentralize a SOP, we need to decentralize a date- $\tau$ optimal path as a date- $\tau$ equilibrium path.

Consider the date- $\tau$ general equilibrium version of the Ramsey model with quasi-hyperbolic discounting. A representative agent solves problem (5), i.e., determines her date- $\tau$ consumer optimum at given sequences of interest and wage rates. A representative firm at each date $t=\tau, \tau+1, \ldots$ solves problem (4) taking as given the interest rates.

The definition of equilibrium is similar to that of the standard Ramsey model (see Section 2.2). A date- $\tau$ equilibrium path (under quasi-hyperbolic discounting) starting from $s_{\tau-1}$ is a sequence $\left\{c_{t}^{* \tau}, s_{t}^{* \tau}, k_{t+1}^{* \tau}, r_{t}^{* \tau}, w_{t}^{* \tau}\right\}_{t=\tau}^{\infty}$, such that 1$)$ the agent maximizes utility correctly foreseeing the prices from the date $\tau$ perspective: $\left\{c_{t}^{* \tau}, s_{t}^{* \tau}\right\}_{t=\tau}^{\infty}$ is a date- $\tau$ consumer optimum starting from $s_{\tau-1}$ at given $\left\{r_{t}^{* \tau}\right\}_{t=\tau}^{\infty}$ and $\left.\left\{w_{t}^{* \tau}\right\}_{t=\tau}^{\infty} ; 2\right)$ at each date prices are equal to the marginal products from the date $\tau$ perspective: $1+r_{t}^{* \tau}=f^{\prime}\left(k_{t}^{* \tau}\right)$ and $\left.w_{t}^{* \tau}=f\left(k_{t}^{* \tau}\right)-f^{\prime}\left(k_{t}^{* \tau}\right) k_{t}^{* \tau} ; 3\right)$ at each date savings are equal to the investment: $s_{t-1}^{* \tau}=k_{t}^{* \tau}$.

Note that date- $\tau$ equilibrium and date- $\tau$ optimal paths are essentially the same - a sequence $\left\{c_{t}^{* \tau}, k_{t+1}^{* \tau}\right\}_{t=\tau}^{\infty}$ extracted from the date- $\tau$ equilibrium path is the date- $\tau$ optimal path (starting from $k_{\tau}=s_{\tau-1}$ ), i.e., the solution to problem (8). It is also evident that the date- $\tau$ equilibrium path differs from the truncation of any previous date equilibrium path, and hence at each date $\tau$ there arises a new equilibrium.

Thus, decentralization of a SOP is a sliding equilibrium path (SEP) obtained by constructing at each date $\tau$ the corresponding date- $\tau$ equilibrium path. It should be emphasized that on a date- $\tau$ equilibrium path, due to naivete, agent's expectations about prices are correct only from the date- $\tau$ perspective. Therefore, the agent recalculates her expectations at each date: on the

\footnotetext{
11 The proof of Claim 1 is based on the fact that under its assumptions, there exists an analytical solution for the $\delta$-optimal path and hence for the date- $\tau$ optimal path under quasi-hyperbolic discounting. This is in stark contrast to the continuous-time Ramsey model, where even for exponential discounting there exists no explicit solution for an optimal path under logarithmic utility (cf. Smith, 2006).
} 
date- $\tau$ equilibrium path, the date- $\tau$ consumer optimum is obtained under expectations $\left\{r_{t}^{* \tau}\right\}_{t=\tau}^{\infty}$ and $\left\{w_{t}^{* \tau}\right\}_{t=\tau}^{\infty}$, while on the date- $\tau^{\prime}$ equilibrium path, date- $\tau^{\prime}$ consumer optimum is obtained under different expectations $\left\{r_{t}^{* \tau^{\prime}}\right\}_{t=\tau^{\prime}}^{\infty}$ and $\left\{w_{t}^{* \tau^{\prime}}\right\}_{t=\tau^{\prime}}^{\infty}$. It turns out that the agent correctly foresees prices on the date- $\tau$ equilibrium path, but she cannot correctly foresee prices on a SEP. The agent has only "pseudo-perfect" foresight in this case, which motivates the following definition.

Definition 4. A sequence $\left\{c_{t}^{\circ}, s_{t}^{\circ}, k_{t+1}^{\circ}, r_{t}^{\circ}, w_{t}^{\circ}\right\}_{t=0}^{\infty}$ is a sliding equilibrium path under pseudo-perfect foresight starting from $s_{-1}^{\circ}$, if

1. Consumption and savings at each date $\tau$ are obtained from the date- $\tau$ equilibrium path, i.e., the date- $\tau$ consumer optimum starting from $s_{\tau-1}^{\circ}$ at given $\left\{r_{t}^{* \tau}\right\}_{t=\tau}^{\infty}$ and $\left\{w_{t}^{* \tau}\right\}_{t=\tau}^{\infty}$;

2. Prices at each date $\tau$ are equal to the corresponding marginal products:

$$
1+r_{\tau}^{\circ}=f^{\prime}\left(k_{\tau}^{\circ}\right), \quad w_{\tau}^{\circ}=f\left(k_{\tau}^{\circ}\right)-f^{\prime}\left(k_{\tau}^{\circ}\right) k_{\tau}^{\circ}
$$

3. Savings at each date $\tau$ are equal to investment:

$$
s_{\tau}^{\circ}=k_{\tau+1}^{\circ}
$$

In fact, a SEP under pseudo-perfect foresight is a sequence $\left\{c_{t}^{\circ}, s_{t}^{\circ}, k_{t+1}^{\circ}, r_{t}^{\circ}, w_{t}^{\circ}\right\}_{t=0}^{\infty}$ such that for each $\tau \geq 0$, consumption, savings, capital stock and prices at date $\tau$ are the elements of the date- $\tau$ equilibrium path starting from $s_{\tau}^{\circ}$ :

$$
\left\{c_{\tau}^{\circ}, s_{\tau}^{\circ}, k_{\tau+1}^{\circ}, r_{\tau}^{\circ}, w_{\tau}^{\circ}\right\}=\left\{c_{\tau}^{* \tau}, s_{\tau}^{* \tau}, k_{\tau+1}^{* \tau}, r_{\tau}^{* \tau}, w_{\tau}^{* \tau}\right\}, \quad \tau=0,1, \ldots
$$

\begin{tabular}{|c|c|c|c|c|c|}
\hline & $\begin{array}{l}\text { Date- } 0 \text { equilibrium } \\
\text { starting from } s_{-1}^{\circ}\end{array}$ & $\begin{array}{l}\text { Date-1 equilibrium } \\
\text { starting from } s_{0}^{* 0}\end{array}$ & $\begin{array}{l}\text { Date-2 equilibrium } \\
\text { starting from } s_{1}^{* 1}\end{array}$ & & $\begin{array}{c}\text { Date- } \tau \text { equilibrium } \\
\text { starting from } s_{\tau-1}^{*(\tau-1)}\end{array}$ \\
\hline$t=0$ & $\begin{array}{c}\mathbf{c}_{0}^{* 0}, \mathbf{s}_{0}^{* 0}, \mathbf{k}_{1}^{* 0} \\
\mathbf{r}_{0}^{* 0}, \mathbf{w}_{0}^{* 0}\end{array}$ & & & & \\
\hline$t=1$ & $\begin{array}{l}c_{1}^{* 0}, s_{1}^{* 0}, k_{2}^{* 0} \\
\quad r_{1}^{* 0}, w_{1}^{* 0}\end{array}$ & $\begin{array}{c}\mathbf{c}_{1}^{* 1}, \mathbf{s}_{1}^{* 1}, \mathbf{k}_{2}^{* 1} \\
\mathbf{r}_{1}^{* 1}, \mathbf{w}_{1}^{* 1}\end{array}$ & & & \\
\hline$t=2$ & $\begin{array}{c}c_{2}^{* 0}, s_{2}^{* 0}, k_{3}^{* 0} \\
r_{2}^{* 0}, w_{2}^{* 0} \\
\end{array}$ & $\begin{array}{c}c_{2}^{* 1}, s_{2}^{* 1}, k_{3}^{* 1} \\
r_{2}^{* 1}, w_{2}^{* 1} \\
\end{array}$ & $\begin{array}{c}c_{2}^{* 2}, s_{2}^{* 2}, k_{3}^{* 2} \\
r_{2}^{* 2}, w_{2}^{* 2} \\
\end{array}$ & & \\
\hline : & : & $\cdot$ & : & & \\
\hline$t=\tau$ & $\begin{array}{c}c_{\tau}^{* 0}, s_{\tau}^{* 0}, k_{\tau+1}^{* 0} \\
r_{\tau}^{* 0}, w_{\tau}^{* 0}\end{array}$ & $\begin{array}{c}c_{\tau}^{* 1}, s_{\tau}^{* 1}, k_{\tau+1}^{* 1} \\
r_{\tau}^{* 1}, w_{\tau}^{* 1}\end{array}$ & $\begin{array}{c}c_{\tau}^{* 2}, s_{\tau}^{* 2}, k_{\tau+1}^{* 2} \\
r_{\tau}^{* 2}, w_{\tau}^{* 2}\end{array}$ & & $\begin{array}{c}\mathbf{c}_{\tau}^{* \tau}, \mathbf{s}_{\tau}^{* \tau}, \mathbf{k}_{\tau+\mathbf{1}}^{* \tau} \\
\mathbf{r}_{\tau}^{* \tau}, \mathbf{w}_{\tau}^{* \tau}\end{array}$ \\
\hline$\vdots$ & $\vdots$ & $\vdots$ & $\vdots$ & $\ddots$ & $\vdots$ \\
\hline
\end{tabular}

A SEP under pseudo-perfect foresight is the main diagonal of the infinite matrix in Table 1.

Table 1: A sliding equilibrium path under pseudo-perfect foresight

Table 1 highlights the fact that a SEP under pseudo-perfect foresight depends on all date- $\tau$ equilibrium paths for $\tau=0,1, \ldots$, and suggests the following interpretation. On a SEP under 
pseudo-perfect foresight, an agent at each date is naive about her time-inconsistent preferences (hence she recalculates her consumer optimum, the first line in each cell of Table 1), and also cannot perfectly foresee prices on a SEP (hence she recalculates the expected prices, the second line in each cell). The agent correctly foresees date- $\tau$ equilibrium prices at each date $\tau$, but cannot take into account that the equilibrium itself will change. Hence the label pseudoperfect foresight which underlines the naivete of the agent.

By construction, a SOP is decentralized as a SEP under pseudo-perfect foresight: a sequence $\left\{c_{t}^{\circ}, k_{t+1}^{\circ}\right\}_{t=0}^{\infty}$ extracted from a SEP under pseudo-perfect foresight is a SOP starting from $k_{0}=$ $s_{-1}$. It immediately follows that a SEP under pseudo-perfect foresight exists and is unique.

It also follows that the question of observational equivalence for SEPs under pseudo-perfect foresight is the same as for SOPs. Formally, a SEP under pseudo-perfect foresight is observationally equivalent to a $\gamma$-optimal path when the corresponding SOP is observationally equivalent to a $\gamma$-optimal path. Therefore, for SEPs under pseudo-perfect foresight observational equivalence holds only in two cases: the stationary case and the case of logarithmic utility and Cobb-Douglas production technology (cf. Claim 1).

Consider the stationary case in more detail. Since a SEP under pseudo-perfect foresight is essentially the same as a SOP, the definition of a stationary sliding equilibrium (SSE) under pseudo-perfect foresight is straightforward.

Definition 5. A tuple $\left\{c^{\circ}, s^{\circ}, k^{\circ}, r^{\circ}, w^{\circ}\right\}$ is a stationary sliding equilibrium under pseudoperfect foresight if the sequence $\left\{c_{t}^{\circ}, s_{t}^{\circ}, k_{t+1}^{\circ}, r_{t}^{\circ}, w_{t}^{\circ}\right\}_{t=0}^{\infty}$, satisfying for each $t=0,1, \ldots$,

$$
c_{t}^{\circ}=c^{\circ}, \quad s_{t}^{\circ}=s^{\circ}, \quad k_{t+1}^{\circ}=k^{\circ}, \quad r_{t}^{\circ}=r^{\circ}, \quad w_{t}^{\circ}=w^{\circ},
$$

is a sliding equilibrium path under pseudo-perfect foresight starting from $k^{\circ}=s^{\circ}$.

Recall that by the definition of SEP under pseudo-perfect foresight, consumption, savings, capital stock and prices, $\left\{c^{\circ}, s^{\circ}, k^{\circ}, r^{\circ}, w^{\circ}\right\}$, are the elements of the associated date- $\tau$ equilibrium path starting from $k^{\circ}$. It is clear that this underlying date- $\tau$ equilibrium path, $\left\{c_{t}^{* \tau}, s_{t}^{* \tau}, k_{t+1}^{* \tau}, r_{t}^{* \tau}, w_{t}^{* \tau}\right\}_{t=\tau}^{\infty}$, which determines a SSE under pseudo-perfect foresight, does not depend on $\tau$ (all columns in Table 1 are identical), and can be described as follows:

$$
\begin{gathered}
\left\{c_{\tau}^{* \tau}, s_{\tau}^{* \tau}, k_{\tau+1}^{* \tau}, r_{\tau}^{* \tau}, w_{\tau}^{* \tau}\right\}=\left\{c^{\circ}, s^{\circ}, k^{\circ}, r^{\circ}, w^{\circ}\right\}, \\
\left\{c_{t}^{* \tau}, s_{t}^{* \tau}, k_{t+1}^{* \tau}, r_{t}^{* \tau}, w_{t}^{* \tau}\right\}_{t=\tau+1}^{\infty}=\delta \text {-equilibrium path starting from } k^{\circ}=s^{\circ} .
\end{gathered}
$$

Note that the sequence of interest rates on this date- $\tau$ equilibrium path, is not constant and has the form $\left\{r^{\circ}, r^{\circ},\left\{r_{t}^{* \tau}\right\}_{t=\tau+2}^{\infty}\right\}$, where the sequence $\left\{r_{t}^{* \tau}\right\}_{t=\tau+2}^{\infty}$ decreases over time and converges to the modified golden rule interest rate for the discount factor $\delta$.

\section{Sliding equilibrium path under perfect foresight}

Now we turn to the central object of our study, a sliding equilibrium path under perfect foresight. Section 4.1 provides a formal definition of a SEP under perfect foresight. In 
Sections 4.2 and 4.3 we analyze the logarithmic utility case and the stationary case respectively. In Section 4.4 we derive the general results concerning the existence of a SEP under perfect foresight, and its observational equivalence. Section 4.5 compares SEPs under pseudo-perfect and perfect foresight.

\subsection{Definition}

As we have seen, the traditional "general equilibrium logic" that says that optimal and equilibrium paths are essentially the same, does not apply in the case of quasi-hyperbolic discounting, because it leads to imperfect foresight. The notion of SEP under pseudo-perfect foresight presupposes that a naive agent at each date recalculates both her consumer optimum and expectations about prices.

Here we introduce an alternative type of expectations in a sliding equilibrium: perfect foresight, as opposed to pseudo-perfect foresight. Under perfect foresight, the agent is only partially naive, as she correctly foresees prices on a SEP, but remains unaware of her time inconsistency and continues to recalculate her consumer optimum. At each date she cannot resist the temptation to consume more than the exponential discounting would prescribe. This leads to the following definition.

Definition 6. A sequence $\left\{c_{t}^{*}, s_{t}^{*}, k_{t+1}^{*}, r_{t}^{*}, w_{t}^{*}\right\}_{t=0}^{\infty}$ is a sliding equilibrium path under perfect foresight starting from $s_{-1}^{*}$, if

1. Consumption and savings at each date $\tau$ are obtained from the date- $\tau$ consumer optimum starting from $s_{\tau-1}^{*}$ at given $\left\{r_{t}^{*}\right\}_{t=\tau}^{\infty}$ and $\left\{w_{t}^{*}\right\}_{t=\tau}^{\infty}$;

2. Prices at each date $\tau$ are equal to the corresponding marginal products:

$$
1+r_{\tau}^{*}=f^{\prime}\left(k_{\tau}^{*}\right), \quad w_{\tau}^{*}=f\left(k_{\tau}^{*}\right)-f^{\prime}\left(k_{\tau}^{*}\right) k_{\tau}^{*}
$$

3. Savings at each date $\tau$ are equal to investment:

$$
s_{\tau}^{*}=k_{\tau+1}^{*} .
$$

A SEP under perfect foresight is an object which is associated with an infinite sequence of corresponding consumer optima, i.e., an infinite sequence of optimization problems of form (5). Indeed, a SEP under perfect foresight is a sequence $\left\{c_{t}^{*}, s_{t}^{*}, k_{t+1}^{*}, r_{t}^{*}, w_{t}^{*}\right\}_{t=0}^{\infty}$ which is characterized as follows:

- there exists a date- 0 consumer optimum starting from $s_{-1}^{*}$ at given $\left\{r_{t}^{*}\right\}_{t=0}^{\infty}$ and $\left\{w_{t}^{*}\right\}_{t=0}^{\infty}$, which we denote by $\left\{c_{t}^{* * 0}, s_{t}^{* * 0}\right\}_{t=0}^{\infty}$, and its first elements are precisely the date-0 consumption and savings on a SEP under perfect foresight: $c_{0}^{* * 0}=c_{0}^{*}$ and $s_{0}^{* * 0}=s_{0}^{*}$;

- there exists a date- 1 consumer optimum starting from $s_{0}^{*}$ at given $\left\{r_{t}^{*}\right\}_{t=1}^{\infty}$ and $\left\{w_{t}^{*}\right\}_{t=1}^{\infty}$ (truncated sequences of sliding equilibrium prices), denoted by $\left\{c_{t}^{* * 1}, s_{t}^{* * 1}\right\}_{t=1}^{\infty}$, and its 
first elements are the date- 1 consumption and savings on a SEP under perfect foresight: $c_{1}^{* * 1}=c_{1}^{*}$ and $s_{1}^{* * 1}=s_{1}^{*}$;

- and so on;

- so that the resulting capital stock sequence $k_{t+1}^{*}=s_{t}^{*}=s_{t}^{* * t}$ precisely determines the sliding equilibrium sequences of interest and wage rates which are correctly expected by the agent when she solves for her consumer optima at each date.

Denote for all $\tau$ and $t, k_{t+1}^{* * \tau}=s_{t}^{* * \tau}$, and, slightly abusing the notation, include the sequence $\left\{k_{t+1}^{* * \tau}\right\}_{t=\tau}^{\infty}$ into the date- $\tau$ consumer optimum. Then a SEP under perfect foresight is the main diagonal of the infinite matrix in Table 2.

\begin{tabular}{|c|c|c|c|c|c|}
\hline & $\begin{array}{c}\text { Date- } 0 \text { optimum at } \\
\text { given }\left\{r_{t}^{*}\right\}_{t=0}^{\infty},\left\{w_{t}^{*}\right\}_{t=0}^{\infty}\end{array}$ & $\begin{array}{c}\text { Date-1 optimum at } \\
\text { given }\left\{r_{t}^{*}\right\}_{t=1}^{\infty},\left\{w_{t}^{*}\right\}_{t=1}^{\infty}\end{array}$ & $\begin{array}{c}\text { Date-2 optimum at } \\
\text { given }\left\{r_{t}^{*}\right\}_{t=2}^{\infty},\left\{w_{t}^{*}\right\}_{t=2}^{\infty}\end{array}$ & & $\begin{array}{c}\text { Date- } \tau \text { optimum at } \\
\text { given }\left\{r_{t}^{*}\right\}_{t=\tau}^{\infty},\left\{w_{t}^{*}\right\}_{t=\tau}^{\infty}\end{array}$ \\
\hline$t=0$ & $\begin{array}{c}\mathbf{c}_{\mathbf{0}}^{* * 0}, \mathbf{s}_{\mathbf{0}}^{* * 0}, \mathbf{k}_{1}^{* * 0} \\
\mathbf{r}_{\mathbf{0}}^{*}, \mathbf{w}_{\mathbf{0}}^{*}\end{array}$ & & & & \\
\hline$t=1$ & $\begin{array}{c}c_{1}^{* * 0}, s_{1}^{* * 0}, k_{2}^{* * 0}, \\
r_{1}^{*}, w_{1}^{*}\end{array}$ & $\begin{array}{c}\mathrm{c}_{1}^{* * 1}, \mathrm{~s}_{1}^{* * 1}, \mathrm{k}_{2}^{* * 1} \\
\mathrm{r}_{1}^{*}, \mathrm{w}_{1}^{*} \\
\end{array}$ & & & \\
\hline$t=2$ & $\begin{array}{c}c_{2}^{* * 0}, s_{2}^{* * 0}, k_{3}^{* * 0} \\
r_{2}^{*}, w_{2}^{*}\end{array}$ & $\begin{array}{c}c_{2}^{* * 1}, s_{2}^{* * 1}, k_{3}^{* * 1} \\
r_{2}^{*}, w_{2}^{*}\end{array}$ & $\begin{array}{c}\mathrm{c}_{2}^{* * 2}, \mathrm{~s}_{2}^{* * 2}, \mathrm{k}_{3}^{* * 2} \\
\mathrm{r}_{2}^{*}, \mathrm{w}_{2}^{*}\end{array}$ & & \\
\hline$\vdots$ & $\vdots$ & $\vdots$ & $\vdots$ & $\ddots$ & \\
\hline$t=\tau$ & $\begin{array}{c}c_{\tau}^{* * 0}, s_{\tau}^{* * 0}, k_{\tau+1}^{* * 0}, \\
r_{\tau}^{*}, w_{\tau}^{*}\end{array}$ & $\begin{array}{c}c_{\tau}^{* * 1}, s_{\tau}^{* * 1}, k_{\tau+1}^{* * 1}, \\
r_{\tau}^{*}, w_{\tau}^{*}\end{array}$ & $\begin{array}{c}c_{\tau}^{* * 2}, s_{\tau}^{* * 2}, k_{\tau+1}^{* * 2}, \\
r_{\tau}^{*}, w_{\tau}^{*}\end{array}$ & $\ldots$ & $\begin{array}{c}\mathbf{c}_{\tau}^{* * \tau}, \mathbf{s}_{\tau}^{* * \tau}, \mathbf{k}_{\tau+\mathbf{1}}^{* * \tau} \\
\mathbf{r}_{\tau}^{*}, \mathbf{w}_{\tau}^{*}\end{array}$ \\
\hline$\vdots$ & $\vdots$ & $\vdots$ & $\vdots$ & $\ddots$ & $\vdots$ \\
\hline
\end{tabular}

Table 2: A sliding equilibrium path under perfect foresight

By comparing Definition 6 with Definition 4, and Table 2 with Table 1, it can be seen that the important difference between SEP under pseudo-perfect foresight and SEP under perfect foresight is the formation of price expectations in the consumer optimum. Under pseudoperfect foresight, the sequences of interest and wage rates expected at date $\tau$ coincide with those realized on the date- $\tau$ equilibrium path, but not on the SEP. Under perfect foresight, the sequences of interest and wage rates expected at date $\tau$ coincide with those realized on the SEP, and hence they are the same for different dates $\tau$ (i.e., in each row of Table 2).

Again, of particular interest is the question of observational equivalence for SEPs under perfect foresight.

Definition 7. A sliding equilibrium path under perfect foresight in the Ramsey model with quasi-hyperbolic discounting starting from $s_{-1}^{*},\left\{c_{t}^{*}, s_{t}^{*}, k_{t+1}^{*}, r_{t}^{*}, w_{t}^{*}\right\}_{t=0}^{\infty}$, is observationally equivalent to a $\gamma$-optimal path, if there exists $\gamma$ for which the sequence $\left\{c_{t}^{*}, k_{t+1}^{*}\right\}_{t=0}^{\infty}$ is a solution to problem (2) starting from $k_{0}^{*}=s_{-1}^{*}$.

\subsection{Logarithmic utility}

It should be emphasized that our Definition 6 of a SEP under perfect foresight and Definition 7 of observational equivalence clarify the ideas behind the Barro (1999) paper. In order to make 
this link and our definitions more transparent, let us consider the particular yet very important case where utility is logarithmic. As in Barro (1999), the following result holds.

Claim 2. Suppose that $u(c)=\ln c$. Then, irrespective of the production technology, a sliding equilibrium path under perfect foresight exists, is unique, and is observationally equivalent to the $\gamma^{*}$-optimal path, where $\gamma^{*}=\frac{\beta \delta}{1-\delta+\beta \delta}$.

Proof. It follows from (6) that when $\rho=1$, the date- $\tau$ consumption in the date- $\tau$ consumer optimum starting from $s_{\tau-1}^{*}$ at given $\left\{r_{t}^{*}\right\}_{t=\tau}^{\infty}$ and $\left\{w_{t}^{*}\right\}_{t=\tau}^{\infty}$, satisfies

$$
c_{\tau}^{* * \tau}=\frac{1-\delta}{1-\delta+\beta \delta} M_{\tau}^{*}
$$

where

$$
M_{\tau}^{*}=\left(1+r_{\tau}^{*}\right) s_{\tau-1}^{*}+w_{\tau}^{*}+\frac{w_{\tau+1}^{*}}{1+r_{\tau+1}^{*}}+\ldots+\frac{w_{\tau+t}^{*}}{\left(1+r_{\tau+1}^{*}\right) \cdots\left(1+r_{\tau+t}^{*}\right)}+\ldots
$$

is the present (date $\tau$ ) value of the expected at date $\tau$ lifetime income. For the formal argument that $M_{\tau}^{*}<\infty$, see the proof of Lemma 2.3 in Appendix C.

By definition, a SEP under perfect foresight starting from $s_{-1}^{*}$ is a sequence $\left\{c_{t}^{*}, s_{t}^{*}, k_{t+1}^{*}, r_{t}^{*}, w_{t}^{*}\right\}_{t=0}^{\infty}$ such that for all $\tau \geq 0$,

$$
\begin{gathered}
c_{\tau}^{*}=\frac{1-\delta}{1-\delta+\beta \delta} M_{\tau}^{*}, \quad k_{\tau+1}^{*}=s_{\tau}^{*}=\left(1+r_{\tau}^{*}\right) s_{\tau-1}^{*}+w_{\tau}^{*}-c_{\tau}^{*}, \\
1+r_{\tau}^{*}=f^{\prime}\left(k_{\tau}^{*}\right), \quad w_{\tau}^{*}=f\left(k_{\tau}^{*}\right)-f^{\prime}\left(k_{\tau}^{*}\right) k_{\tau}^{*} .
\end{gathered}
$$

It follows that lifetime incomes expected at dates $\tau$ and $\tau+1$ are linked:

$$
M_{\tau}^{*}-\left(1+r_{\tau}^{*}\right) s_{\tau-1}^{*}-w_{\tau}^{*}=\frac{M_{\tau+1}^{*}}{1+r_{\tau+1}^{*}}-s_{\tau}^{*}
$$

and hence $M_{\tau+1}^{*}=\left(1+r_{\tau+1}^{*}\right)\left(M_{\tau}^{*}-c_{\tau}^{*}\right)$. Therefore,

$$
\begin{aligned}
c_{\tau+1}^{*}=\frac{1-\delta}{1-\delta+\beta \delta} M_{\tau+1}^{*} & =\frac{1-\delta}{1-\delta+\beta \delta}\left(1+r_{\tau+1}^{*}\right)\left(M_{\tau}^{*}-c_{\tau}^{*}\right) \\
& =\left(1+r_{\tau+1}^{*}\right) c_{\tau}^{*}-\frac{1-\delta}{1-\delta+\beta \delta}\left(1+r_{\tau+1}^{*}\right) c_{\tau}^{*}=\frac{\beta \delta}{1-\delta+\beta \delta}\left(1+r_{\tau+1}^{*}\right) c_{\tau}^{*} .
\end{aligned}
$$

Thus on a SEP under perfect foresight, the consumption levels at two adjacent dates are linked via the following "first-order conditions":

$$
c_{t+1}^{*}=\frac{\beta \delta}{1-\delta+\beta \delta}\left(1+r_{t+1}^{*}\right) c_{t}^{*}, \quad t=0,1, \ldots
$$

Now it is clear that the sequence $\left\{c_{t}^{*}, k_{t+1}^{*}\right\}_{t=0}^{\infty}$ extracted from a SEP under perfect foresight is the $\gamma^{*}$-optimal path, where $\gamma^{*}=\frac{\beta \delta}{1-\delta+\beta \delta}$, and hence observational equivalence holds.

Three comments about Claim 2 are in order. First, it can be compared to the results of 
Barro (1999). Barro's argument implies that the agent reconsiders her consumption path at each instant of time, but she does not recalculate her expectations and acts as if she correctly foresees prices taking into account that the equilibrium will change. Thus Barro (1999) implicitly assumes a naive agent and considers a SEP under perfect foresight in terms of our Definition 6 , and our result about observational equivalence under logarithmic utility naturally confirms his findings.

Second, Krusell et al. (2002) prove observational equivalence for a sophisticated path in the Ramsey model with quasi-hyperbolic discounting, logarithmic utility and Cobb-Douglas production technology, and obtain the same formula for the equivalent discount factor $\gamma^{*}$. However, Krusell et al. (2002) consider a sophisticated agent, while we consider a naive agent; and they consider only Cobb-Douglas specification, while our result holds irrespective of the production technology (and the formula for $\gamma^{*}$ does not depend on the production technology).

Third, Claim 2 establishes the link between observational equivalence and a controlled comparison of discount functions (see, e.g., Myerson et al., 2001; Caliendo and Findley, 2014). Note that the equivalent discount factor $\gamma^{*}$ is such that the exponential $\gamma^{*}$ discounting provides the same degree of overall impatience as the quasi-hyperbolic $\beta-\delta$ discounting:

$$
1+\gamma^{*}+\left(\gamma^{*}\right)^{2}+\ldots+\left(\gamma^{*}\right)^{t}+\ldots=1+\beta \delta+\beta \delta^{2}+\ldots+\beta \delta^{t}+\ldots
$$

Under logarithmic utility, controlling for overall impatience implies that the paths of consumption and capital are observationally equivalent under both discounting methods.

\subsection{Stationary sliding equilibrium}

Before turning to the general results about transitional paths, let us consider a stationary sliding equilibrium (SSE) under perfect foresight.

Definition 8. A tuple $\left\{c^{*}, s^{*}, k^{*}, r^{*}, w^{*}\right\}$ is a stationary sliding equilibrium under perfect foresight if the sequence $\left\{c_{t}^{*}, s_{t}^{*}, k_{t+1}^{*}, r_{t}^{*}, w_{t}^{*}\right\}_{t=0}^{\infty}$, satisfying for each $t=0,1, \ldots$,

$$
c_{t}^{*}=c^{*}, \quad s_{t}^{*}=s^{*}, \quad k_{t+1}^{*}=k^{*}, \quad r_{t}^{*}=r^{*}, \quad w_{t}^{*}=w^{*},
$$

is a sliding equilibrium path under perfect foresight starting from $s^{*}$.

Recall that by the definition of SEP under perfect foresight, consumption and savings $\left\{c^{*}, s^{*}\right\}$ are obtained from the associated date- $\tau$ consumer optimum starting from $s^{*}$ at given constant interest rate $r^{*}$ and wage rate $w^{*}$. It is clear that this underlying date- $\tau$ consumer optimum, which determines a SSE under perfect foresight, does not depend on $\tau$.

An important difference between SSE under pseudo-perfect foresight and SSE under perfect foresight lies in the formation of expectations about prices in the consumer optimum. As we have seen, consumption and savings on a SSE under pseudo-perfect foresight, $\left\{c^{\circ}, s^{\circ}\right\}$, are the first elements in the consumer optimum starting from $s^{\circ}$ at given $\left\{r^{\circ}, r^{\circ},\left\{r_{t}^{* \tau}\right\}_{t=\tau+2}^{\infty}\right\}$ and $\left\{w^{\circ}, w^{\circ},\left\{w_{t}^{* \tau}\right\}_{t=\tau+2}^{\infty}\right\}$. At the same time, consumption and savings on a SSE under perfect 
foresight, $\left\{c^{*}, s^{*}\right\}$, are the first elements in the consumer optimum starting from $s^{*}$ at given $\left\{r^{*}, r^{*}, r^{*}, \ldots\right\}$ and $\left\{w^{*}, w^{*}, w^{*}, \ldots\right\}$.

The following theorem maintains that a SSE under perfect foresight exists, is unique and there is always observational equivalence.

Theorem 1. A stationary sliding equilibrium under perfect foresight exists, is unique and is observationally equivalent to the stationary $\gamma^{*}$-optimum, where $\gamma^{*}$ satisfies

$$
\gamma^{*}=\frac{\left(\gamma^{*}\right)^{\frac{1}{\rho}}-(\beta \delta)^{\frac{1}{\rho}}}{\delta^{\frac{1}{\rho}}-(\beta \delta)^{\frac{1}{\rho}}}
$$

Proof. Take $r^{*}>0$ and some $w^{*}>0$. Let $\left\{c_{t}^{* * \tau}\right\}_{t=\tau}^{\infty}$ be consumption in a date- $\tau$ consumer optimum starting from $s^{*}$ at given constant interest rate $r^{*}$ and wage rate $w^{*}$, i.e., the solution to problem (5) for $\left\{r_{t}\right\}_{t=\tau}^{\infty}=\left\{r^{*}, r^{*}, \ldots\right\}$ and $\left\{w_{t}\right\}_{t=\tau}^{\infty}=\left\{w^{*}, w^{*}, \ldots\right\}$. The following lemma characterizes this consumer optimum.

Lemma 1.1. Suppose that $\delta\left(1+r^{*}\right)^{1-\rho}<1$. A date- $\tau$ consumer optimum starting from $s^{*}$ at given $r^{*}$ and $w^{*}$ exists, is unique, and the date- $\tau$ consumption satisfies

$$
c_{\tau}^{* * \tau}=\frac{1-\delta^{\frac{1}{\rho}}\left(1+r^{*}\right)^{\frac{1-\rho}{\rho}}}{1-\delta^{\frac{1}{\rho}}\left(1+r^{*}\right)^{\frac{1-\rho}{\rho}}+(\beta \delta)^{\frac{1}{\rho}}\left(1+r^{*}\right)^{\frac{1-\rho}{\rho}}} \cdot \frac{1+r^{*}}{r^{*}} \cdot\left(r^{*} s^{*}+w^{*}\right) .
$$

Proof. See Appendix B.

By the definition of SSE under perfect foresight,

$$
c_{\tau}^{* * \tau}=c^{*}=\left(1+r^{*}\right) s^{*}+w^{*}-s^{*}=r^{*} s^{*}+w^{*} .
$$

It now follows from (10) that the interest rate in a SSE under perfect foresight satisfies the following equation:

$$
1+r^{*}=\frac{1-\delta^{\frac{1}{\rho}}\left(1+r^{*}\right)^{\frac{1-\rho}{\rho}}+(\beta \delta)^{\frac{1}{\rho}}\left(1+r^{*}\right)^{\frac{1-\rho}{\rho}}}{(\beta \delta)^{\frac{1}{\rho}}\left(1+r^{*}\right)^{\frac{1-\rho}{\rho}}},
$$

which can be rewritten as

$$
\frac{1}{1+r^{*}}=\frac{\left(\frac{1}{1+r^{*}}\right)^{\frac{1}{\rho}}-(\beta \delta)^{\frac{1}{\rho}}}{\delta^{\frac{1}{\rho}}-(\beta \delta)^{\frac{1}{\rho}}}
$$

The following lemma maintains that there exists a unique solution to equation (11), and it is compatible with the existence of a date- $\tau$ consumer optimum.

Lemma 1.2. There is a unique solution $r^{*}$ to equation (11), which satisfies $\delta\left(1+r^{*}\right)^{1-\rho}<1$.

Proof. See Appendix B.

It follows that the tuple $\left\{c^{*}, s^{*}, k^{*}, r^{*}, w^{*}\right\}$, where $r^{*}$ is the solution to equation (11), and

$$
s^{*}=k^{*}=\left(f^{\prime}\right)^{-1}\left(1+r^{*}\right), \quad w^{*}=f\left(k^{*}\right)-f^{\prime}\left(k^{*}\right) k^{*}, \quad c^{*}=r^{*} s^{*}+w^{*},
$$

is the unique SSE under perfect foresight. 
Furthermore, denote $\gamma^{*}=\frac{1}{1+r^{*}}$. It is clear that since $r^{*}$ satisfies (11), $\gamma^{*}$ satisfies (9). Since $f^{\prime}\left(k^{*}\right)=1 / \gamma^{*}$, it follows that $\left\{c^{*}, k^{*}\right\}$ is a stationary optimum in the standard Ramsey model with the discount factor $\gamma^{*}$. Therefore, a SSE under perfect foresight is observationally equivalent to a stationary $\gamma^{*}$-optimum.

It can be checked (see Appendix B) that the stationary equivalent discount factor $\gamma^{*}$ lies in between the short-run discount factor $\beta \delta$ and the long-run discount factor $\delta\left(\beta \delta<\gamma^{*}<\delta\right)$, and is monotonically increasing both in $\beta$ and in $\delta$. Furthermore, $\gamma^{*}$ is monotonically decreasing in $\rho$, and when $\rho$ tends to $0, \gamma^{*}$ tends to $\delta$.

It also follows directly from Claim 2 that for $u(c)=\ln c$, a SSE under perfect foresight is observationally equivalent to the stationary $\gamma^{*}$-optimum where $\gamma^{*}=\frac{\beta \delta}{1-\delta+\beta \delta}$. It is easily seen that this $\gamma^{*}$ is the solution to equation (9) for $\rho=1$. Thus, in the stationary case with logarithmic utility, Theorem 1 and Claim 2 yield the same result, as they should.

\subsection{General results}

Next, we provide a number of analytical results about the full transitional paths for general isoelastic utility. The following theorem proves the existence of a SEP under perfect foresight.

Theorem 2. For any $s_{-1}^{*}>0$ there exists a sliding equilibrium path under perfect foresight starting from $s_{-1}^{*}$.

Proof. We prove the existence of a SEP under perfect foresight in two steps. First, we consider a SEP under perfect foresight in the finite horizon model and show that for any $T>0$ there exists a finite $T$-horizon SEP. Second, we construct a candidate for a SEP in the infinite horizon model by applying some kind of diagonalization procedure to the sequence of finite $T$-horizon SEPs, and then show that this candidate is indeed a SEP under perfect foresight in the infinite horizon model.

Fix a finite horizon $T>0$. For any date $\tau=0, \ldots, T$, consider the following $T$-horizon date- $\tau$ problem:

$$
\begin{array}{ll}
\max _{\left\{c_{t}\right\}} & u\left(c_{\tau}\right)+\beta \sum_{t=\tau+1}^{T+1} \delta^{t-\tau} u\left(c_{t}\right), \\
\text { s. t. } & c_{\tau}+\frac{c_{\tau+1}}{1+r_{\tau+1}}+\ldots+\frac{c_{T+1}}{\left(1+r_{\tau+1}\right) \cdots\left(1+r_{T+1}\right)} \\
& \leq f\left(k_{\tau}\right)+\frac{w_{\tau+1}}{1+r_{\tau+1}}+\ldots+\frac{w_{T+1}}{\left(1+r_{\tau+1}\right) \cdots\left(1+r_{T+1}\right)}, \\
& c_{t} \geq 0, \quad t=\tau, \ldots, T+1,
\end{array}
$$

where the initial capital stock $k_{\tau}>0$ and sequences of interest rates $\left\{r_{t+1}\right\}_{t=\tau}^{T}$ and wage rates $\left\{w_{t+1}\right\}_{t=\tau}^{T}$ are taken as given by the agent.

Similarly to the infinite horizon case (cf. Section 2.2), we call the sequence $\left\{c_{t}^{\tau}, s_{t}^{\tau}\right\}_{t=\tau}^{T+1} \mathbf{a} T$ horizon date- $\tau$ consumer optimum starting from $k_{\tau}$ at given $\left\{r_{t+1}\right\}_{t=\tau}^{T}$ and $\left\{w_{t+1}\right\}_{t=\tau}^{T}$ 
if $\left\{c_{t}^{\tau}\right\}_{t=\tau}^{T+1}$ is the solution to problem (12), and $\left\{s_{t}^{\tau}\right\}_{t=\tau}^{T+1}$ is given recursively by

$$
s_{\tau}^{\tau}=f\left(k_{\tau}\right)-c_{\tau}^{\tau}, \quad s_{t}^{\tau}=\left(1+r_{t}\right) s_{t-1}^{\tau}+w_{t}-c_{t}^{\tau}, \quad t=\tau+1, \ldots, T+1
$$

A finite $T$-horizon SEP under perfect foresight is formally defined as follows.

Definition 9. A sequence $\left\{c_{t}^{*}(T), s_{t}^{*}(T), k_{t+1}^{*}(T), r_{t+1}^{*}(T), w_{t+1}^{*}(T)\right\}_{t=0}^{T}$ is a $T$-horizon sliding equilibrium path under perfect foresight starting from $s_{-1}^{*}=k_{0}^{*}$ if

1. Consumption and savings at date $\tau$ are the elements of the T-horizon date- $\tau$ consumer optimum starting from $k_{\tau}^{*}(T)$ at given $\left\{r_{t+1}^{*}(T)\right\}_{t=\tau}^{T}$ and $\left\{w_{t+1}^{*}(T)\right\}_{t=\tau}^{T}$;

2. Prices at each date are equal to the corresponding marginal products: for $t=1, \ldots, T+1$,

$$
1+r_{t}^{*}(T)=f^{\prime}\left(k_{t}^{*}(T)\right), \quad w_{t}^{*}(T)=f\left(k_{t}^{*}(T)\right)-f^{\prime}\left(k_{t}^{*}(T)\right) k_{t}^{*}(T)
$$

3. Savings at each date are equal to investment: for $t=0, \ldots, T$,

$$
s_{t}^{*}(T)=k_{t+1}^{*}(T) .
$$

This definition is completely analogous to Definition 6 of the infinite-horizon SEP under perfect foresight. Our notation highlights the fact that we consider the finite $T$-horizon case.

The following lemma establishes the existence of a $T$-horizon SEP under perfect foresight.

Lemma 2.1. There exists a T-horizon sliding equilibrium path under perfect foresight starting from any $s_{-1}^{*}=k_{0}^{*}>0$.

Proof. For the formal proof, see Appendix C. The idea of the proof is to construct a continuous mapping from some compact convex set of capital stock sequences $\left\{k_{t+1}\right\}_{t=0}^{T}$ to itself, and obtain a fixed point of this mapping, $\left\{k_{t+1}^{*}\right\}_{t=0}^{T}$. We then consider a $T$-horizon date- $\tau$ consumer optima starting from $k_{\tau}^{*}$ at given $\left\{r_{t+1}^{*}\right\}_{t=\tau}^{T}$ and $\left\{w_{t+1}^{*}\right\}_{t=\tau}^{T}$, where the interest and wage rates are determined by the obtained fixed point. We study the first elements of these optima and show that the obtained fixed point $\left\{k_{t+1}^{*}\right\}_{t=0}^{T}$ in fact determines a $T$-horizon SEP under perfect foresight.

Importantly enough, the sequence of capital stocks on a $T$-horizon SEP under perfect foresight is bounded from both below and above. To define the lower bounds, let $\left\{\underline{c}_{t}(T), \underline{k}_{t+1}(T)\right\}_{t=0}^{T+1}$ be a solution to the following problem given $\underline{k}_{0}=k_{0}^{*}$ :

$$
\begin{aligned}
\max _{\left\{c_{t}, k_{t+1}\right\}} & \sum_{t=0}^{T+1}(\beta \delta)^{t} u\left(c_{t}\right), \\
\text { s. t. } & c_{t}+k_{t+1}=f\left(k_{t}\right), \quad c_{t} \geq 0, \quad k_{t+1} \geq 0, \quad t=0, \ldots, T+1 .
\end{aligned}
$$

To define the upper bounds, let $\left\{\bar{c}_{t}(T), \bar{k}_{t+1}(T)\right\}_{t=0}^{T+1}$ be a solution to the following problem given 
$\bar{k}_{0}=k_{0}^{*}$ :

$$
\max _{\left\{c_{t}, k_{t+1}\right\}} \sum_{t=0}^{T+1} \delta^{t} u\left(c_{t}\right),
$$

s. t. $\quad c_{t}+k_{t+1}=f\left(k_{t}\right), \quad c_{t} \geq 0, \quad k_{t+1} \geq 0, \quad t=0, \ldots, T+1$.

The following lemma shows that the sequence of capital stocks on a $T$-horizon SEP under perfect foresight is bounded from below by the sequence of capital stocks $\left\{\underline{k}_{t+1}(T)\right\}_{t=0}^{T}$, and is bounded from above by the sequence of capital stocks $\left\{\bar{k}_{t+1}(T)\right\}_{t=0}^{T}$.

Lemma 2.2. For all $T=1,2, \ldots$, and for all $t=0, \ldots, T$,

$$
\underline{k}_{t+1}(T)<k_{t+1}^{*}(T)<\bar{k}_{t+1}(T) .
$$

Proof. See Appendix C.

Now consider the sequence $\left[\left\{k_{t+1}^{*}(T)\right\}_{t=0}^{T}\right]_{T=1,2, \ldots}$, whose elements are the sequences of capital stocks on the $T$-horizon SEPs under perfect foresight starting from the same $s_{-1}^{*}=k_{0}^{*}$, for increasing horizons $T=1,2, \ldots$.

Let us apply the following procedure to the sequence $\left[\left\{k_{t+1}^{*}(T)\right\}_{t=0}^{T}\right]_{T=1,2, \ldots}$. At the first step of our procedure, consider the sequence $\left\{k_{1}^{*}(T)\right\}_{T=1,2, \ldots}$, take a cluster point $k_{1}^{*}$ of this sequence and extract a subsequence $\left\{T_{0 n}\right\}_{n=1}^{\infty}$ from $\{T\}_{T=1,2, \ldots}$ such that $\left\{k_{1}^{*}\left(T_{0 n}\right)\right\}_{n=1}^{\infty}$ converges to $k_{1}^{*}$. At the second step, consider the sequence $\left\{k_{2}^{*}\left(T_{0 n}\right)\right\}_{n=1}^{\infty}$, take a cluster point $k_{2}^{*}$ of this sequence and extract a subsequence $\left\{T_{1 n}\right\}_{n=1}^{\infty}$ from the sequence $\left\{T_{0 n}\right\}_{n=1}^{\infty}$ such that $T_{11}>1$ and $\left\{k_{2}^{*}\left(T_{1 n}\right)\right\}_{n=1}^{\infty}$ converges to $k_{2}^{*}$. This procedure continues ad infinitum.

Finally, consider the sequence $\left\{c_{t}^{*}, s_{t}^{*}, k_{t+1}^{*}, r_{t+1}^{*}, w_{t+1}^{*}\right\}_{t=0}^{\infty}$, where $\left\{k_{t+1}^{*}\right\}_{t=0}^{\infty}$ is obtained by the diagonal procedure described above and for all $\tau=0,1 \ldots$,

$$
\begin{aligned}
& c_{\tau}^{*}=f\left(k_{\tau}^{*}\right)-k_{\tau+1}^{*}, \quad s_{\tau}^{*}=k_{\tau+1}^{*}, \\
& 1+r_{\tau}^{*}=f^{\prime}\left(k_{\tau}^{*}\right), \quad w_{\tau}^{*}=f\left(k_{\tau}^{*}\right)-f^{\prime}\left(k_{\tau}^{*}\right) k_{\tau}^{*} \text {. }
\end{aligned}
$$

The following lemma ensures that this sequence is a SEP under perfect foresight.

Lemma 2.3. The sequence $\left\{c_{t}^{*}, s_{t}^{*}, k_{t+1}^{*}, r_{t+1}^{*}, w_{t+1}^{*}\right\}_{t=0}^{\infty}$ is a sliding equilibrium path under perfect foresight starting from $s_{-1}^{*}=k_{0}^{*}$.

Proof. See Appendix C.

Thus we obtain a sliding equilibrium path under perfect foresight and prove the theorem.

Though a SEP under perfect foresight always exists, observational equivalence is not a general phenomenon. The following theorem proves that the already considered cases of logarithmic utility and stationary sliding equilibria are the only cases where SEP under perfect foresight is observationally equivalent to some $\gamma$-optimal path.

Theorem 3. A sliding equilibrium path under perfect foresight starting from $s_{-1}^{*} \neq s^{*}$ is observationally equivalent to a $\gamma$-optimal path if and only if $\rho=1$. 
Proof. Suppose that a SEP under perfect foresight, $\left\{c_{t}^{*}, s_{t}^{*}, k_{t+1}^{*}, r_{t}^{*}, w_{t}^{*}\right\}_{t=0}^{\infty}$, is observationally equivalent to some $\gamma$-optimal path. Then it follows from Theorem 1 that the equivalent discount factor must be $\gamma^{*}$, and $\left\{c_{t}^{*}, s_{t}^{*}, k_{t+1}^{*}, r_{t}^{*}, w_{t}^{*}\right\}_{t=0}^{\infty}$ converges to the SSE under perfect foresight $\left\{c^{*}, s^{*}, k^{*}, r^{*}, w^{*}\right\}$ characterized in Theorem 1 .

Let $\Delta_{t+1}$ be given by

$$
\Delta_{t+1}=\delta^{\frac{1}{\rho}}\left(1+r_{t+2}^{*}\right)^{\frac{1-\rho}{\rho}}+\delta^{\frac{2}{\rho}}\left(1+r_{t+2}^{*}\right)^{\frac{1-\rho}{\rho}}\left(1+r_{t+3}^{*}\right)^{\frac{1-\rho}{\rho}}+\ldots
$$

It is easily seen that

$$
\Delta_{t+1}=\delta^{\frac{1}{\rho}}\left(1+r_{t+2}^{*}\right)^{\frac{1-\rho}{\rho}}\left(1+\Delta_{t+2}\right)
$$

The following lemma shows that the consumption levels at two adjacent dates on a SEP under perfect foresight are linked via some kind of "first-order conditions".

Lemma 3.1. Let $\left\{c_{t}^{*}, s_{t}^{*}, k_{t+1}^{*}, r_{t}^{*}, w_{t}^{*}\right\}_{t=0}^{\infty}$ be a SEP under perfect foresight. For all $t=0,1, \ldots$,

$$
c_{t+1}^{*}=c_{t}^{*}\left(\beta \delta\left(1+r_{t+1}^{*}\right)\right)^{\frac{1}{\rho}} \frac{1+\Delta_{t+1}}{1+\beta^{\frac{1}{\rho}} \Delta_{t+1}} .
$$

Proof. See Appendix D.

At the same time, due to observational equivalence,

$$
c_{t+1}^{*}=c_{t}^{*}\left(\gamma^{*}\left(1+r_{t+1}^{*}\right)\right)^{\frac{1}{\rho}} .
$$

By comparing (20) and (21), we obtain that for all $t=0,1, \ldots$,

$$
\left(\frac{\gamma^{*}}{\beta \delta}\right)^{\frac{1}{\rho}}=\frac{1+\Delta_{t+1}}{1+\beta^{\frac{1}{\rho}} \Delta_{t+1}} \Leftrightarrow\left(\frac{\gamma^{*}}{\beta \delta}\right)^{\frac{1}{\rho}}+\left(\frac{\gamma^{*}}{\delta}\right)^{\frac{1}{\rho}} \Delta_{t+1}=1+\Delta_{t+1} \Leftrightarrow \Delta_{t+1}=\frac{\left(\gamma^{*}\right)^{\frac{1}{\rho}}-(\beta \delta)^{\frac{1}{\rho}}}{(\beta \delta)^{\frac{1}{\rho}}-\left(\beta \gamma^{*}\right)^{\frac{1}{\rho}}} .
$$

Therefore, the value of $\Delta_{t+1}$ is constant over time. Using (19) and (9), we can restate this condition in terms of interest rates as follows:

$$
\left(1+r_{t+2}^{*}\right)^{\frac{1-\rho}{\rho}}=\frac{1}{\left(\gamma^{*}\right)^{\frac{1}{\rho}}} \cdot \frac{\left(\gamma^{*}\right)^{\frac{1}{\rho}}-(\beta \delta)^{\frac{1}{\rho}}}{\delta^{\frac{1}{\rho}}-(\beta \delta)^{\frac{1}{\rho}}}=\left(\gamma^{*}\right)^{1-\frac{1}{\rho}}
$$

or

$$
\left(\gamma^{*}\left(1+r_{t+2}^{*}\right)\right)^{\frac{1-\rho}{\rho}}=1, \quad t=0,1, \ldots
$$

Clearly, (22) holds only in the following two cases. First, $\rho=1$, which is the logarithmic utility case, and it was already established in Claim 2 that observational equivalence holds. Second, $r_{t}^{*}=r^{*}$, which is the stationary case with $s_{-1}^{*}=s^{*}$. Therefore, in the Ramsey model with quasi-hyperbolic discounting and isoelastic utility function, a SEP under perfect foresight starting from $s_{-1}^{*} \neq s^{*}$ cannot be observationally equivalent to an optimal path in the Ramsey model with exponential discounting unless $\rho=1$.

The intuition behind Theorem 3 is as follows. Due to the fact that under perfect foresight the expected prices are not recalculated and remain the same at each date, on a SEP under 
perfect foresight the consumption levels at two adjacent dates are linked and satisfy the "firstorder conditions" (20). These conditions are compatible with the first-order conditions in the standard Ramsey model if and only if either an interest rate is constant or utility is logarithmic.

Thus, a SEP under perfect foresight is observationally equivalent to some $\gamma$-optimal path only in two cases. First, in the case of logarithmic utility considered in Claim 2 where the equivalent discount factor is given by $\gamma^{*}=\frac{\beta \delta}{1-\delta+\beta \delta}$. Second, in the case of a SSE under perfect foresight considered in Theorem 1 where the equivalent discount factor $\gamma^{*}$ satisfies equation (9). In all other cases, a SEP under perfect foresight cannot be observationally equivalent to any $\gamma$-optimal path.

\subsection{Comparison}

Finally, we compare the properties of SEPs under pseudo-perfect and perfect foresight in terms of saving rates, long-run capital stocks and consumption.

As we have seen, Claims 1 and 2 suggest that already in the simplest case where $u(c)=\ln c$ and $f(k)=k^{\alpha}$, SEPs under pseudo-perfect and perfect foresight are two completely different objects. Recall that by Claim 1, the SEP under pseudo-perfect foresight is observationally equivalent to the $\gamma^{\circ}$-optimal path, where $\gamma^{\circ}=\frac{\beta \delta}{1-\alpha \delta+\alpha \beta \delta}$ (which depends on the capital elasticity of output in the Cobb-Douglas production technology). At the same time, by Claim 2, the SEP under perfect foresight is observationally equivalent to the $\gamma^{*}$-optimal path, where $\gamma^{*}=\frac{\beta \delta}{1-\delta+\beta \delta}$ (which does not depend on the production technology). Comparing the equivalent discount factors, it is easily seen that $\beta \delta<\gamma^{\circ}<\gamma^{*}<\delta$. Thus, the equivalent exponential discount factor is higher under perfect foresight than under pseudo-perfect foresight.

It is well known that for $u(c)=\ln c$ and $f(k)=k^{\alpha}$, the saving rate on the $\gamma$-optimal path is constant and given by $\mathrm{s}=\alpha \gamma$. Therefore, in the considered case the saving rate is always higher under perfect foresight than under pseudo-perfect foresight: $\mathbf{s}^{*}=\alpha \gamma^{*}>\alpha \gamma^{\circ}=\mathbf{s}^{\circ}$. It follows that if the two economies start from the same initial condition, then capital stock at each date on the SEP under perfect foresight is higher than capital stock on the SEP under pseudo-perfect foresight. Consequently, while initially consumption is higher on the SEP under pseudo-perfect foresight, starting from some date, the SEP under perfect foresight provides higher consumption. Therefore, the stationary capital stock and consumption level are higher under perfect foresight than under pseudo-perfect foresight. Loosely speaking, more rationality means more consumption in the stationary state.

To illustrate these results, in Figure 1 we compare consumption (left panel) and the capital stock (right panel) on three different paths: SEP under pseudo-perfect foresight, SEP under perfect foresight, and date-0 optimal path, i.e., the path obtained under the strategy of precommitment, which would be realized if the agent was able to implement her decisions made at date 0 . Note that on the committed path the consumption profile may be non-monotonic, though both the initial and long-run consumption levels on the committed path are higher than those on the SEP under perfect foresight.

The result about stationary states can be generalized - it also holds for SSEs when intertemporal elasticity of substitution in consumption is sufficiently high. The comparison of 

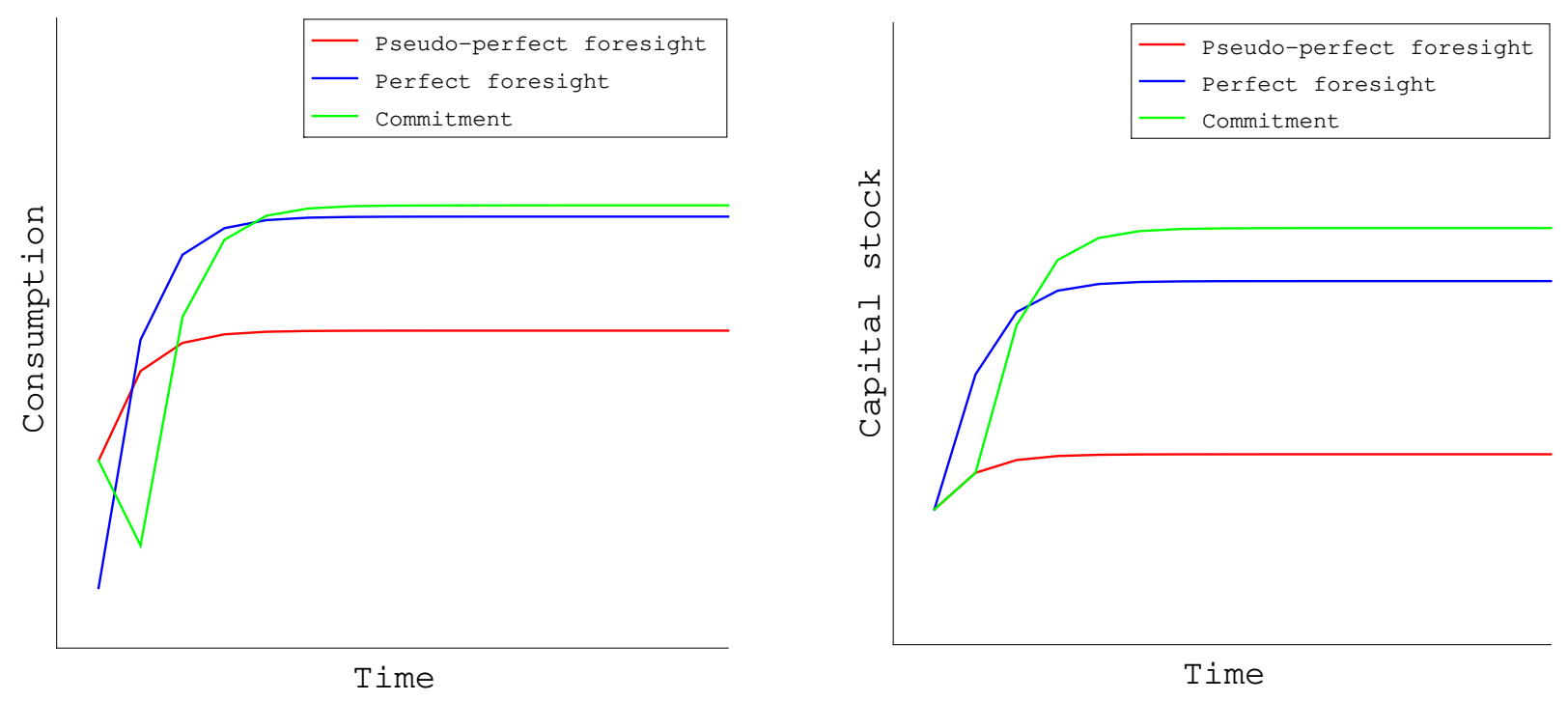

Figure 1: Comparison of different equilibrium paths $\left(\alpha=0.3, \beta=0.5, \delta=0.9, k_{0}=0.05\right)$.

consumer optima at given constant and non-constant sequences of interest and wage rates, allows us to compare SSEs under pseudo-perfect and perfect foresight. The following theorem maintains that when $0<\rho \leq 1$, a stationary capital stock is higher under perfect foresight than under pseudo-perfect foresight, irrespective of a production technology.

Theorem 4. Let $\left\{c^{*}, s^{*}, k^{*}, r^{*}, w^{*}\right\}$ be a stationary sliding equilibrium under perfect foresight, and $\left\{c^{\circ}, s^{\circ}, k^{\circ}, r^{\circ}, w^{\circ}\right\}$ be a stationary sliding equilibrium under pseudo-perfect foresight. If preferences are such that $0<\rho \leq 1$, then

$$
c^{*}>c^{\circ}, \quad s^{*}>s^{\circ}, \quad k^{*}>k^{\circ}, \quad r^{*}<r^{\circ}, \quad w^{*}>w^{\circ} .
$$

Proof. For the formal proof, see Appendix E. We provide here only the sketch of the proof. The idea is to assume the opposite and obtain a contradiction.

Suppose that $k^{*} \leq k^{\circ}$. It then follows that $r^{\circ} \leq r^{*}$ and $w^{\circ} \geq w^{*}$. Let $c\left(r^{\circ}, w^{\circ}\right)$ be the date- $\tau$ consumption in the date- $\tau$ consumer optimum starting from $s^{\circ}$ at given constant interest rate $r^{\circ}$ and wage rate $w^{\circ}$.

On the one hand, since $c^{\circ}$ is consumption in a stationary sliding equilibrium (under pseudoperfect foresight), it can be checked that $k^{\circ} \geq k^{*}$ implies $c\left(r^{\circ}, w^{\circ}\right) \geq c^{\circ}$. However, on the other hand, when $0<\rho \leq 1$, it is easily seen from (6) that the date- $\tau$ consumption in the date- $\tau$ consumer optimum starting from $s_{\tau-1}$ at given $\left\{r_{t}\right\}_{t=\tau}^{\infty}$ and $\left\{w_{t}\right\}_{t=\tau}^{\infty}$ is monotonically increasing in $w_{t}$ and decreasing in $r_{t}$ for all $t \geq \tau+1$. Therefore, $c\left(r^{\circ}, w^{\circ}\right)$ is lower than the date- $\tau$ consumption level on the date- $\tau$ equilibrium path starting from $s^{\circ}$, which is $c^{\circ}$.

This contradiction shows that for $0<\rho \leq 1$ we must have $k^{*}>k^{\circ}$. It then follows that $s^{*}>s^{\circ}, r^{*}<r^{\circ}, w^{*}>w^{\circ}$, and $c^{*}=f\left(k^{*}\right)-k^{*}>f\left(k^{\circ}\right)-k^{\circ}=c^{\circ}$.

We conjecture that the above result also holds for $\rho>1$. We have made many simulations with different values of $\beta$ and $\delta$, and our simulations support this conjecture. 


\section{Conclusion}

In this paper we consider the Ramsey model with quasi-hyperbolic discounting and emphasize the following main points. First, quasi-hyperbolic discounting involves time inconsistency, irrespective of whether we consider optimal or equilibrium paths. We assume that an agent cannot commit to future actions and is naive about her time inconsistency. To describe the behavior of an agent who recalculates her consumer optimum at each date, we introduce sliding optimal and sliding equilibrium paths. Under exponential discounting, sliding paths correspond to the usual optimal and equilibrium paths.

Second, sliding equilibrium paths critically depend on expectations, and we distinguish between pseudo-perfect foresight and perfect foresight. Under pseudo-perfect foresight, the expected sequences of interest and wage rates at each date $\tau$ coincide with those realized on the "temporary" date- $\tau$ equilibrium path, while under perfect foresight, the expected sequences of interest and wage rates at each date coincide with those realized on the sliding path. Thus, under pseudo-perfect foresight an agent at each date is naive both about her future preferences (revising her consumer optimum) and future prices (revising her expectations about prices). In contrast, under perfect foresight an agent is only partially naive - she recalculates her consumer optimum, but correctly foresees prices on the sliding path.

Third, in general, there is no observational equivalence for sliding equilibrium paths, i.e., a consumption path on a sliding equilibrium path coincides with that in a standard Ramsey model only in a number of very special cases. We note that observational equivalence for sliding equilibrium paths under pseudo-perfect foresight holds only in two cases: the stationary case and the case with logarithmic utility and Cobb-Douglas production technology. We also prove the existence of a sliding equilibrium path under perfect foresight for a general isoelastic utility function, and show that observational equivalence holds if and only if either an interest rate is constant or utility is logarithmic. In all other cases, a sliding equilibrium path under perfect foresight cannot coincide with any optimal path in a standard Ramsey model.

Fourth, even in the simplest cases sliding equilibrium paths under pseudo-perfect and perfect foresight differ. We compare sliding equilibria under pseudo-perfect and perfect foresight in terms of saving rates for the stationary case and for the case with logarithmic utility and Cobb-Douglas production technology. We show that perfect foresight implies a higher capital stock and a higher consumption level than pseudo-perfect foresight. Loosely speaking, less naivete leads to higher capital accumulation in the long run.

There are still several open questions to be addressed by future studies. Of special interest is the dynamics of sliding equilibrium paths under perfect foresight, i.e., whether they converge to stationary sliding equilibrium under perfect foresight. Also, the conditions under which observational equivalence holds for sliding equilibrium paths under pseudo-perfect foresight are yet to be fully characterized.

Furthermore, our research can be extended in a number of ways. It is of interest to compare sliding equilibrium paths under pseudo-perfect and perfect foresight in terms of welfare, which is not a trivial task, as welfare criteria under time inconsistency are not clearly defined. Another possible direction of future research is to consider a sliding equilibrium path where agents are 
heterogeneous in their time preferences or differ in their degree of naivety (i.e., some agents have pseudo-perfect foresight, and some perfect foresight).

We hope that this study will help to clarify the nature of quasi-hyperbolic discounting and contribute to the debate on economic dynamics with time-inconsistent preferences.

\section{References}

[1] Ainslie G. (1992). Picoeconomics. Cambridge: Cambridge University Press.

[2] Angeletos G.-M., Laibson D., Repetto A., Tobacman J., Weinberg S. (2001). The Hyperbolic Consumption Model: Calibration, Simulation, and Empirical Evaluation. Journal of Economic Perspectives, 15 (3), pp. 47-68.

[3] Barro R. J. (1999). Ramsey Meets Laibson in the Neoclassical Growth Model. Quarterly Journal of Economics, 114 (4), pp. 1125-1152.

[4] Bernheim B. D., Ray D., Yeltekin Ş. (2015). Poverty and Self-control. Econometrica, 83 (5), pp. 1877-1911.

[5] Borissov K. (2013). Growth and Distribution in a Model with Endogenous Time Preferences and Borrowing Constraints. Mathematical Social Sciences, 66, pp. 117-128.

[6] Caliendo F. N., Findley T. S. (2014). Discount Functions and Self-control Problems. Economics Letters, 122 (3), pp. 416-419.

[7] Cao D., Werning I. (2018). Saving and Dissaving with Hyperbolic Discounting. Econometrica, 86 (3), pp. 805-857.

[8] Cass D. (1965). Optimum Growth in an Aggregative Model of Capital Accumulation. Review of Economic Studies, 32 (3), pp. 233-240.

[9] DellaVigna S. (2009). Psychology and Economics: Evidence from the Field. Journal of Economic Literature, 47 (2), pp. 315-372.

[10] Drugeon J.-P., Wigniolle B. (2019). On a Simple Equilibrium with Heterogeneous Quasihyperbolic Discounting Agents. Revue d'Economie Politique, 129 (5), pp. 715-740.

[11] Ekeland I., Lazrak A. (2010). The Golden Rule When Preferences Are Time Inconsistent. Mathematics and Financial Economics, 4 (1), pp. 29-55.

[12] Farzin Y. H., Wendner R. (2014). The Time Path of the Saving Rate: Hyperbolic Discounting and Short-Term Planning. MPRA Paper No. 54614.

[13] Findley T. S., Caliendo F. N. (2014). Interacting Mechanisms of Time Inconsistency. Journal of Economic Psychology, 41, pp. 68-76.

[14] Frederick S., Loewenstein G., O’Donoghue T. (2002). Time Discounting and Time Preference: A Critical Review. Journal of Economic Literature, 40 (2), pp. 351-401. 
[15] Goldman S. M. (1968). Optimal Growth and Continual Planning Revision. Review of Economic Studies, 35 (2), pp. 145-154.

[16] Goldman S. M. (1980). Consistent Plans. Review of Economic Studies, 47 (3), pp. 533-537.

[17] Harris C., Laibson D. (2001). Dynamic Choices of Hyperbolic Consumers. Econometrica, 69 (4), pp. 935-957.

[18] Kaganovich M. (1985). Efficiency of Sliding Plans in a Linear Model with Time-dependent Technology. Review of Economic Studies, 52 (4), pp. 691-702.

[19] Koopmans T. (1965). On the Concept of Optimal Economic Growth. In The Econometric Approach to Development Planning. Amsterdam: North Holland.

[20] Krusell P., Kuruşçu B., Smith A. A. (2002). Equilibrium Welfare and Government Policy with Quasi-geometric Discounting. Journal of Economic Theory, 105 (1), pp. 42-72.

[21] Krusell P., Smith A. A. (2003). Consumption-savings Decisions with Quasi-geometric Discounting. Econometrica, 71 (1), pp. 365-375.

[22] Laibson D. I. (1996). Hyperbolic Discount Functions, Undersaving, and Savings Policy. NBER Working Paper No. 5635.

[23] Laibson D. I. (1997). Golden Eggs and Hyperbolic Discounting. Quarterly Journal of Economics, 112 (2), pp. 443-478.

[24] Loewenstein G., Prelec D. (1992). Anomalies in Intertemporal Choice: Evidence and an Interpretation. Quarterly Journal of Economics, 107 (2), pp. 573-597.

[25] Maliar L., Maliar S. (2006). The Neoclassical Growth Model with Heterogeneous Quasigeometric Consumers. Journal of Money, Credit and Banking, 38 (3), pp. 635-654.

[26] Myerson J., Green L., Warusawitharana M. (2001). Area under the Curve as a Measure of Discounting. Journal of the Experimental Analysis of Behavior, 76, pp. 235-243.

[27] O’Donoghue T., Rabin M. (2001). Choice and Procrastination. Quarterly Journal of Economics, 116 (1), pp. 121-160.

[28] Peleg B., Yaari M. E. (1973). On the Existence of a Consistent Course of Action When Tastes Are Changing. Review of Economic Studies, 40 (3), pp. 391-401.

[29] Phelps E. S., Pollak R. A. (1968). On Second-best National Saving and Game-equilibrium Growth. Review of Economic Studies, 35 (2), pp. 185-199.

[30] Pollak R. A. (1968). Consistent Planning. Review of Economic Studies, 35 (2), pp. 201-208.

[31] Ramsey F. P. (1928). A Mathematical Theory of Saving. Economic Journal, 38, pp. 543559. 
[32] Smith W. T. (2006). A Closed Form Solution to the Ramsey Model. Contributions in Macroeconomics, 6 (1), pp. 1-27.

[33] Sorger G. (2004). Consistent Planning under Quasi-geometric Discounting. Journal of Economic Theory, 118 (1), pp. 118-129.

[34] Sorger G. (2007). Time-preference and Commitment. Journal of Economic Behavior and Organization, 62 (4), pp. 556-578.

[35] Strotz R. H. (1955). Myopia and Inconsistency in Dynamic Utility Maximization. Review of Economic Studies, 23 (3), pp. 165-180.

\section{A Sliding optimal paths: A characterizarion}

Let $V_{\gamma}(k)$ be the value function of problem (2), i.e., the solution to the Bellman equation:

$$
V_{\gamma}(k)=\max _{0 \leq k^{\prime} \leq f(k)} u\left(f(k)-k^{\prime}\right)+\gamma V_{\gamma}\left(k^{\prime}\right)
$$

The associated policy function $g_{\gamma}(k)$ is implicitly given by the following functional equation:

$$
u^{\prime}\left(f(k)-g_{\gamma}(k)\right)=\gamma V_{\gamma}^{\prime}\left(g_{\gamma}(k)\right)
$$

Thus the $\gamma$-optimal path in the standard Ramsey model is fully determined by value function $V_{\gamma}(k)$ or policy function $g_{\gamma}(k)$.

Let us now characterize the first step in problem (8) in terms of dynamic programming. Since the continuation of the date- $\tau$ optimal path is the $\delta$-optimal path, it follows that at date $\tau$ the quasi-hyperbolic planner solves the following problem:

$$
\max _{0 \leq k^{\prime} \leq f(k)} u\left(f(k)-k^{\prime}\right)+\beta \delta V_{\delta}\left(k^{\prime}\right) .
$$

Therefore, the policy function $h(k)$ which is the solution to the following functional equation:

$$
u^{\prime}(f(k)-h(k))=\beta \delta V_{\delta}^{\prime}(h(k)),
$$

describes the first step on the date- $\tau$ optimal path, and hence determines a SOP. It follows that a SOP is a sequence $\left\{c_{t}^{\circ}, k_{t+1}^{\circ}\right\}_{t=0}^{\infty}$ such that for $t=0,1, \ldots$,

$$
c_{t}^{\circ}=f\left(k_{t}^{\circ}\right)-h\left(k_{t}^{\circ}\right), \quad k_{t+1}^{\circ}=h\left(k_{t}^{\circ}\right) .
$$

Clearly, a SOP under $\beta-\delta$ discounting is observationally equivalent to some $\gamma$-optimal path if and only if $h(k)=g_{\gamma}(k)$ for all $k$, which, using (A.2) and (A.3), can be written as

$$
\gamma V_{\gamma}^{\prime}(k)=\beta \delta V_{\delta}^{\prime}(k), \quad \forall k
$$


Therefore, the question of observational equivalence for SOPs boils down to the properties of a value function of the standard Ramsey model.

It is very hard to expect that the derivative of a value function, $\gamma V_{\gamma}^{\prime}(k)$, simply scales when a discount factor changes, i.e., that equation (A.4) holds. Therefore, to the best of our knowledge, a SOP under quasi-hyperbolic discounting is observationally equivalent to a $\gamma$-optimal path either for a stationary sliding optimum or for logarithmic utility and Cobb-Douglas production technology. Indeed, when $u(c)=\ln c$ and $f(k)=k^{\alpha}$, there is a closed-form expression for the value function $V_{\gamma}(k)$ which solves equation (A.1):

$$
V_{\gamma}(k)=\frac{1}{1-\gamma}\left(\ln (1-\alpha \gamma)+\frac{\alpha \gamma}{1-\alpha \gamma} \ln (\alpha \gamma)\right)+\frac{\alpha}{1-\alpha \gamma} \ln k
$$

and the associated policy function is given by

$$
g_{\gamma}(k)=\alpha \gamma k^{\alpha}
$$

Then equation (A.3) which determines the policy function $h(k)$ associated with a SOP takes the form

$$
\frac{1}{k^{\alpha}-h(k)}=\beta \delta V_{\delta}^{\prime}(h(k)) \Leftrightarrow \frac{1}{k^{\alpha}-h(k)}=\frac{\alpha \beta \delta}{1-\alpha \delta} \frac{1}{h(k)}
$$

and hence

$$
h(k)=\frac{\alpha \beta \delta}{1-\alpha \delta+\alpha \beta \delta} k^{\alpha} .
$$

Thus the policy function $h(k)$ coincides with the policy function $g_{\gamma^{\circ}}(k)$ associated with the $\gamma^{\circ}$-optimal path for $\gamma^{\circ}=\frac{\beta \delta}{1-\alpha \delta+\alpha \beta \delta}$. Therefore, a SOP under quasi-hyperbolic discounting is observationally equivalent to this $\gamma^{\circ}$-optimal path, which proves Claim 1.

As for the stationary sliding optimum, it is always observationally equivalent to a stationary $\gamma^{\circ}$-optimum for $\gamma^{\circ}=1 / f^{\prime}\left(k^{\circ}\right)$. Let us show that $\gamma^{\circ}<\delta$, which is equivalent to $k^{\circ}<k^{\delta}$, where $k^{\delta}$ is the modified golden rule capital stock for the discount factor $\delta$. Indeed, $k^{\circ}$ is the solution to equation (A.3) for $h(k)=k$, which for an isoelastic utility function takes the form

$$
f(k)-k=\left(\beta \delta V_{\delta}^{\prime}(k)\right)^{-\frac{1}{\rho}}
$$

Consider the functions $L(k)=f(k)-k$ and $R(k)=\frac{1}{\left(\beta \delta V_{\delta}^{\prime}(k)\right)^{\frac{1}{\rho}}}$. Clearly, $L(k)$ monotonically increases for $k<k^{\delta}$, is concave, and $L(0)=0$. At the same time, since the Bellman function $V_{\delta}(k)$ is concave, we have

$$
R^{\prime}(k)=\frac{1}{\rho(\beta \delta)^{\frac{1}{\rho}}} \frac{\left|V_{\delta}^{\prime \prime}(k)\right|}{\left(V_{\delta}^{\prime}(k)\right)^{1+\frac{1}{\rho}}}>0
$$

Thus $R(k)$ also monotonically increases for $k<k^{\delta}$, and $R(0)=0$. When $\beta=1$, the solution to (A.5) is $k^{\delta}$. As $\beta$ decreases, $R(k)$ shifts upward, and hence the capital stock which solves equation (A.5) decreases. This means that for $\beta<1$, we have $k^{\circ}<k^{\delta}$, i.e., $\gamma^{\circ}<\delta$. 


\section{B Proof of Theorem 1}

\section{B.1 Proof of Lemma 1.1}

Since $\frac{1}{1+r^{*}}<1$, the right-hand side of the budget constraint in problem (5) under constant interest rate $r^{*}$ and wage rate $w^{*}$ is finite:

$$
\begin{array}{r}
\left(1+r^{*}\right) s^{*}+w^{*}+\frac{w^{*}}{1+r^{*}}+\frac{w^{*}}{\left(1+r^{*}\right)^{2}}+\ldots=\left(1+r^{*}\right) s^{*}+w^{*}\left(1+\frac{1}{1+r^{*}}+\frac{1}{\left(1+r^{*}\right)^{2}}+\ldots\right) \\
=\left(1+r^{*}\right) s^{*}+\frac{w^{*}}{1-\frac{1}{1+r^{*}}}=\frac{1+r^{*}}{r^{*}}\left(r^{*} s^{*}+w^{*}\right)<+\infty
\end{array}
$$

It now follows from (6) that

$$
c_{\tau}^{* * \tau}=\frac{\frac{1+r^{*}}{r^{*}}\left(r^{*} s^{*}+w^{*}\right)}{1+(\beta \delta)^{\frac{1}{\rho}}\left(1+r^{*}\right)^{\frac{1-\rho}{\rho}}+\left(\beta \delta^{2}\right)^{\frac{1}{\rho}}\left(1+r^{*}\right)^{\frac{2(1-\rho)}{\rho}}+\ldots+\left(\beta \delta^{t}\right)^{\frac{1}{\rho}}\left(1+r^{*}\right)^{\frac{t(1-\rho)}{\rho}}+\ldots} .
$$

Since $\delta\left(1+r^{*}\right)^{1-\rho}<1$, the sum of the infinite series in the denominator is finite:

$$
\begin{gathered}
1+(\beta \delta)^{\frac{1}{\rho}}\left(1+r^{*}\right)^{\frac{1-\rho}{\rho}}+\left(\beta \delta^{2}\right)^{\frac{1}{\rho}}\left(1+r^{*}\right)^{\frac{2(1-\rho)}{\rho}}+\ldots+\left(\beta \delta^{t}\right)^{\frac{1}{\rho}}\left(1+r^{*}\right)^{\frac{t(1-\rho)}{\rho}}+\ldots \\
=1+(\beta \delta)^{\frac{1}{\rho}}\left(1+r^{*}\right)^{\frac{1-\rho}{\rho}}\left(1+\left(\delta\left(1+r^{*}\right)^{1-\rho}\right)^{\frac{1}{\rho}}+\left(\delta\left(1+r^{*}\right)^{1-\rho}\right)^{\frac{2}{\rho}}+\ldots\right) \\
=1+\frac{(\beta \delta)^{\frac{1}{\rho}}\left(1+r^{*}\right)^{\frac{1-\rho}{\rho}}}{1-\delta^{\frac{1}{\rho}}\left(1+r^{*}\right)^{\frac{1-\rho}{\rho}}}=\frac{1-\delta^{\frac{1}{\rho}}\left(1+r^{*}\right)^{\frac{1-\rho}{\rho}}+(\beta \delta)^{\frac{1}{\rho}}\left(1+r^{*}\right)^{\frac{1-\rho}{\rho}}}{1-\delta^{\frac{1}{\rho}}\left(1+r^{*}\right)^{\frac{1-\rho}{\rho}}}
\end{gathered}
$$

Thus a date- $\tau$ consumer optimum at given $r^{*}$ and $w^{*}$ exists and is unique. In this optimum, $c_{\tau}^{* * \tau}$ is given by (10), which proves the lemma.

\section{B.2 Proof of Lemma 1.2}

The interest rate on a SSE under perfect foresight solves

$$
1+r^{*}=\frac{1-\delta^{\frac{1}{\rho}}\left(1+r^{*}\right)^{\frac{1-\rho}{\rho}}+(\beta \delta)^{\frac{1}{\rho}}\left(1+r^{*}\right)^{\frac{1-\rho}{\rho}}}{(\beta \delta)^{\frac{1}{\rho}}\left(1+r^{*}\right)^{\frac{1-\rho}{\rho}}}
$$

Rearranging the above equation, we get

$$
\begin{aligned}
& (\beta \delta)^{\frac{1}{\rho}}\left(1+r^{*}\right)^{\frac{1-\rho}{\rho}}\left(1+r^{*}\right)=1-\delta^{\frac{1}{\rho}}\left(1+r^{*}\right)^{\frac{1-\rho}{\rho}}+(\beta \delta)^{\frac{1}{\rho}}\left(1+r^{*}\right)^{\frac{1-\rho}{\rho}} \\
& \Leftrightarrow\left(\delta^{\frac{1}{\rho}}-(\beta \delta)^{\frac{1}{\rho}}\right)\left(1+r^{*}\right)^{\frac{1-\rho}{\rho}}=1-(\beta \delta)^{\frac{1}{\rho}}\left(1+r^{*}\right)^{\frac{1}{\rho}} \Leftrightarrow \frac{1}{1+r^{*}}=\frac{\left(\frac{1}{1+r^{*}}\right)^{\frac{1}{\rho}}-(\beta \delta)^{\frac{1}{\rho}}}{\delta^{\frac{1}{\rho}}-(\beta \delta)^{\frac{1}{\rho}}},
\end{aligned}
$$

and hence $r^{*}$ is the solution to equation (11).

Denote $\gamma^{*}=\frac{1}{1+r^{*}}$. It is clear that equations (11) and (9) are essentially the same. Let us show that there exists a unique solution to equation (9), and this solution satisfies $\delta<\left(\gamma^{*}\right)^{1-\rho}$, i.e., $\delta\left(1+r^{*}\right)^{1-\rho}<1$. This would mean that the interest rate in a SSE under perfect foresight 
exists, is unique and is compatible with the existence of a date- $\tau$ consumer optimum.

Consider the functions $L(\gamma)=\gamma$, and $R(\gamma)=\frac{\gamma^{\frac{1}{\rho}}-(\beta \delta)^{\frac{1}{\rho}}}{\delta^{\frac{1}{\rho}}-(\beta \delta)^{\frac{1}{\rho}}}$. Both $L(\gamma)$ and $R(\gamma)$ are monotonically increasing in $\gamma$, and

$$
R(\beta \delta)=0<\beta \delta=L(\beta \delta), \quad R(\delta)=1>\delta=L(\delta)
$$

Since $R(\gamma)$ is strictly convex for $\rho<1$, linear for $\rho=1$, and strictly concave for $\rho>1$, it follows that for any $\rho$ there is a unique value $\gamma^{*}$ such that $L\left(\gamma^{*}\right)=R\left(\gamma^{*}\right)$. This $\gamma^{*}$ is the solution to equation (9), and it is clear that $\beta \delta<\gamma^{*}<\delta$.

Now it can be directly checked that $\delta<\left(\gamma^{*}\right)^{1-\rho}$ :

$\delta<\left(\gamma^{*}\right)^{1-\rho} \Leftrightarrow \gamma^{*}<\frac{\left(\gamma^{*}\right)^{\frac{1}{\rho}}}{\delta^{\frac{1}{\rho}}} \Leftrightarrow \frac{\left(\gamma^{*}\right)^{\frac{1}{\rho}}-(\beta \delta)^{\frac{1}{\rho}}}{\delta^{\frac{1}{\rho}}-(\beta \delta)^{\frac{1}{\rho}}}<\frac{\left(\gamma^{*}\right)^{\frac{1}{\rho}}}{\delta^{\frac{1}{\rho}}} \Leftrightarrow \beta^{\frac{1}{\rho}}\left(\left(\gamma^{*}\right)^{\frac{1}{\rho}}-\delta^{\frac{1}{\rho}}\right)<0 \Leftrightarrow \gamma^{*}<\delta$

which completes the proof of the lemma.

Let us also characterize the dependence of $\gamma^{*}$ on the parameters of preferences. It is easily checked that $\partial R(\gamma) / \partial \beta<0$ and $\partial R(\gamma) / \partial \delta<0$, which means that for larger $\beta$ or $\delta, R(\gamma)$ crosses $L(\gamma)=\gamma$ for higher values of $\gamma$. Therefore, $\gamma^{*}$ is increasing in both $\beta$ and $\delta$.

Note also that for $\gamma>\beta \delta$,

$$
\frac{\partial R(\gamma)}{\partial \rho}=\frac{1}{\rho^{2}} \cdot \frac{1-\left(\frac{\gamma}{\delta}\right)^{\frac{1}{\rho}}}{1-\beta^{\frac{1}{\rho}}} \cdot\left(\frac{\ln \frac{\delta}{\gamma}}{\left(\frac{\delta}{\gamma}\right)^{\frac{1}{\rho}}-1}-\frac{\ln \frac{1}{\beta}}{\left(\frac{1}{\beta}\right)^{\frac{1}{\rho}}-1}\right)>0
$$

since in this case $1 / \beta>\delta / \gamma$ and the function $\ln x /\left(x^{1 / \rho}-1\right)$ is monotonically decreasing. Therefore, the higher is $\rho$, the earlier $R(\gamma)$ crosses $L(\gamma)=\gamma$, and hence $\gamma^{*}$ is decreasing in $\rho$.

\section{Proof of Theorem 2}

\section{C.1 A T-horizon SEP under perfect foresight}

Fix a finite horizon $T>0$ and consider a $T$-horizon date- $\tau$ consumer optimum. Similarly to the infinite horizon case (cf. equation (6)), it is easily checked that for all $\tau=0, \ldots, T$, date- $\tau$ consumption level on a $T$-horizon date- $\tau$ consumer optimum starting from $k_{\tau}$ at given $\left\{r_{t+1}\right\}_{t=\tau}^{T}$ and $\left\{w_{t+1}\right\}_{t=\tau}^{T}$ satisfies

$$
c_{\tau}^{\tau}=\frac{f\left(k_{\tau}\right)+\frac{w_{\tau+1}}{1+r_{\tau+1}}+\ldots+\frac{w_{T+1}}{\left(1+r_{\tau+1}\right) \cdots\left(1+r_{T+1}\right)}}{1+(\beta \delta)^{\frac{1}{\rho}}\left(1+r_{\tau+1}\right)^{\frac{1-\rho}{\rho}}+\ldots+\left(\beta \delta^{T+1-\tau}\right)^{\frac{1}{\rho}}\left(1+r_{\tau+1}\right)^{\frac{1-\rho}{\rho}} \cdots\left(1+r_{T+1}\right)^{\frac{1-\rho}{\rho}}} .
$$

Also, similarly to the infinite horizon case, a $T$-horizon SEP under perfect foresight is associated with $T+1$ optimization problems of the form (12), i.e., with the sequence of the corresponding $T$-horizon consumer optima. More precisely, a $T$-horizon SEP under perfect foresight starting from $s_{-1}^{*}=k_{0}^{*}$ is a sequence $\left\{c_{t}^{*}, s_{t}^{*}, k_{t+1}^{*}, r_{t+1}^{*}, w_{t+1}^{*}\right\}_{t=0}^{T}$ characterized as follows: 
- there exists a $T$-horizon date-0 consumer optimum starting from $k_{0}^{*}$ given $\left\{r_{t+1}^{*}\right\}_{t=0}^{T}$ and $\left\{w_{t+1}^{*}\right\}_{t=0}^{T}$, which we denote by $\left\{c_{t}^{* * 0}, s_{t}^{* * 0}\right\}_{t=0}^{T+1}$, and its first elements are precisely the date-0 consumption and savings on a $T$-horizon SEP under perfect foresight: $c_{0}^{* * 0}=c_{0}^{*}$ and $s_{0}^{* * 0}=s_{0}^{*}$

- there exists a $T$-horizon date-1 consumer optimum starting from $k_{1}^{*}$ at given $\left\{r_{t+1}^{*}\right\}_{t=1}^{T}$ and $\left\{w_{t+1}^{*}\right\}_{t=1}^{T}$, (truncated sequences of sliding equilibrium interest and wage rates), denoted by $\left\{c_{t}^{* * 1}, s_{t}^{* * 1}\right\}_{t=1}^{T+1}$, and its first elements are the date- 1 consumption and savings on a $T$-horizon SEP under perfect foresight: $c_{1}^{* * 1}=c_{1}^{*}$ and $s_{1}^{* * 1}=s_{1}^{*}$;

- and so on, till the $T$-horizon date- $T$ consumer optimum starting from $k_{T}^{*}$ given $r_{T+1}^{*}$ and $w_{T+1}^{*}$, denoted by $\left\{c_{t}^{* * T}, s_{t}^{* * T}\right\}_{t=T}^{T+1}$, whose first elements are the date- $T$ consumption and savings on a $T$-horizon SEP under perfect foresight: $c_{T}^{* * T}=c_{T}^{*}$ and $s_{T}^{* * T}=s_{T}^{*}$;

- and it turns out that the resulting sliding equilibrium capital stock sequence $\left\{k_{t+1}^{*}\right\}_{t=0}^{T}=$ $\left\{s_{t}^{* * t}\right\}_{t=0}^{T}$ determines precisely those interest and wage rates which were correctly expected by the agent when she solved for her consumer optima at each date.

\section{C.2 Proof of Lemma 2.1}

\section{Step 1. Lower bounds for capital.}

Let $\left\{\underline{c}_{t}(T), \underline{k}_{t+1}(T)\right\}_{t=0}^{T+1}$ be a solution to problem (14). Let also $\underline{s}_{-1}=\underline{k}_{0}$, and for $t=$ $0, \ldots, T+1$

$$
\underline{s}_{t}(T)=\underline{k}_{t+1}(T), \quad 1+\underline{r}_{t}(T)=f^{\prime}\left(\underline{k}_{t}(T)\right), \quad \underline{w}_{t}(T)=f\left(\underline{k}_{t}(T)\right)-f^{\prime}\left(\underline{k}_{t}(T)\right) \underline{k}_{t}(T) .
$$

It is well known that the sequence $\left\{\underline{c}_{t}(T), \underline{s}_{t}(T), \underline{k}_{t+1}(T), \underline{r}_{t}(T), \underline{w}_{t}(T)\right\}_{t=0}^{T+1}$ is a $T$-horizon equilibrium path starting from $\underline{s}_{-1}=\underline{k}_{0}$ in the standard Ramsey model with a discount factor $\beta \delta$. In what follows, a finite horizon $T$ is fixed, so we shall omit the notation " $(T)$ " as long as it does not lead to confusion.

In particular, $\left\{\underline{c}_{t}\right\}_{t=0}^{T+1}$ is a solution to the problem

$$
\begin{aligned}
\max _{\left\{c_{t}\right\}} & \sum_{t=0}^{T+1}(\beta \delta)^{t} u\left(c_{t}\right), \\
\text { s. t. } & c_{0}+\frac{c_{1}}{1+\underline{r}_{1}}+\ldots+\frac{c_{T+1}}{\left(1+\underline{r}_{1}\right) \cdots\left(1+\underline{r}_{T+1}\right)} \\
& \leq f\left(\underline{k}_{0}\right)+\frac{\underline{w}_{1}}{1+\underline{r}_{1}}+\ldots+\frac{\underline{w}_{T+1}}{\left(1+\underline{r}_{1}\right) \cdots\left(1+\underline{r}_{T+1}\right)}, \\
& c_{t} \geq 0, \quad t=0, \ldots, T+1,
\end{aligned}
$$

and the following observation holds.

Claim C.1. For all $\tau=0, \ldots, T$,

$$
\underline{c}_{\tau}=\frac{f\left(\underline{k}_{\tau}\right)+\frac{\underline{w}_{\tau+1}}{1+\underline{r}_{\tau+1}}+\ldots+\frac{\underline{w}_{T+1}}{\left(1+\underline{r}_{\tau+1}\right) \cdots\left(1+\underline{r}_{T+1}\right)}}{1+(\beta \delta)^{\frac{1}{\rho}}\left(1+\underline{r}_{\tau+1}\right)^{\frac{1-\rho}{\rho}}+\ldots+(\beta \delta)^{\frac{T+1-\tau}{\rho}}\left(1+\underline{r}_{\tau+1}\right)^{\frac{1-\rho}{\rho}} \cdots\left(1+\underline{r}_{T+1}\right)^{\frac{1-\rho}{\rho}} .}
$$


Proof. The solution to problem (C.2), $\left\{\underline{c}_{t}\right\}_{t=0}^{T+1}$, satisfies the following first-order conditions:

$$
\underline{c}_{t+1}=\left(\beta \delta\left(1+\underline{r}_{t+1}\right)\right)^{\frac{1}{\rho}} \underline{c}_{t}, \quad t=0, \ldots, T .
$$

Substituting (C.4) into the budget constraint in (C.2) which holds as equality, we get

$$
\begin{aligned}
\underline{c}_{0}\left(1+(\beta \delta)^{\frac{1}{\rho}}\left(1+\underline{r}_{1}\right)^{\frac{1-\rho}{\rho}}+\ldots+(\beta \delta)^{\frac{T+1-\tau}{\rho}}\right. & \left.\left(1+\underline{r}_{1}\right)^{\frac{1-\rho}{\rho}} \cdots\left(1+\underline{r}_{T+1}\right)^{\frac{1-\rho}{\rho}}\right) \\
& =f\left(\underline{k}_{0}\right)+\frac{\underline{w}_{1}}{1+\underline{r}_{1}}+\ldots+\frac{\underline{w}_{T+1}}{\left(1+\underline{r}_{1}\right) \cdots\left(1+\underline{r}_{T+1}\right)},
\end{aligned}
$$

which proves (C.3) for $\tau=0$.

To prove it for $\tau=1, \ldots, T$, it is sufficient to note that the corresponding budget constraint holds for any $\tau$ :

$$
\begin{aligned}
\underline{c}_{\tau}+\frac{\underline{c}_{\tau+1}}{1+\underline{r}_{\tau+1}}+\ldots+\frac{\underline{c}_{T+1}}{\left(1+\underline{r}_{\tau+1}\right)} & \cdots\left(1+\underline{r}_{T+1}\right) \\
& =f\left(\underline{k}_{\tau}\right)+\frac{\underline{w}_{\tau+1}}{1+\underline{r}_{\tau+1}}+\ldots+\frac{\underline{w}_{T+1}}{\left(1+\underline{r}_{\tau+1}\right) \cdots\left(1+\underline{r}_{T+1}\right)}
\end{aligned}
$$

and repeat the argument.

\section{Step 2. Constructing a fixed point.}

Consider the following set consisting of bounded sequences of length $T+1$ :

$$
\mathcal{S}_{T}=\left\{\left\{k_{t+1}\right\}_{t=0}^{T} \mid \underline{k}_{1} \leq k_{1} \leq f\left(k_{0}^{*}\right), \quad \text { and } \quad \underline{k}_{t+1} \leq k_{t+1} \leq f\left(k_{t}\right), \quad t=1, \ldots, T\right\}
$$

Clearly, $\mathcal{S}_{T}$ is a non-empty compact convex set. Now, given $\left\{k_{t+1}\right\}_{t=0}^{T} \in \mathcal{S}_{T}$, consider the sequence $\left\{\tilde{k}_{t+1}\right\}_{t=0}^{T}$ constructed recursively as follows:

$$
\begin{gathered}
\tilde{k}_{1}=\max \left\{\underline{k}_{1}, f\left(k_{0}^{*}\right)-c_{0}^{0}\right\}, \\
\tilde{k}_{\tau+1}=\max \left\{\underline{k}_{\tau+1}, f\left(\tilde{k}_{\tau}\right)-c_{\tau}^{\tau}\right\}, \quad \tau=1, \ldots, T,
\end{gathered}
$$

where $c_{\tau}^{\tau}$ is given by (C.1) for $1+r_{t}=f^{\prime}\left(k_{t}\right)$, and $w_{t}=f\left(k_{t}\right)-f^{\prime}\left(k_{t}\right) k_{t}$. In other words, $c_{\tau}^{\tau}$ is date- $\tau$ consumption on a $T$-horizon date- $\tau$ consumer optimum starting from $k_{\tau}$ at given interest and wage rates determined by the sequence $\left\{k_{t+1}\right\}_{t=\tau}^{T}$.

By construction, we have $\tilde{k}_{t+1} \geq \underline{k}_{t+1}$ for all $t=0, \ldots, T$. Moreover, since $c_{\tau}^{\tau} \geq 0$ and $\underline{k}_{t+1} \leq f\left(\underline{k}_{t}\right)$, it also follows that

$$
\tilde{k}_{1} \leq f\left(k_{0}^{*}\right), \quad \tilde{k}_{t+1} \leq f\left(\tilde{k}_{t}\right), \quad t=1, \ldots, T
$$

Therefore, $\left\{\tilde{k}_{t+1}\right\}_{t=0}^{T} \in \mathcal{S}_{T}$.

Thus we have a continuous mapping from a compact convex set $\mathcal{S}_{T}$ to itself which maps the sequence $\left\{k_{t+1}\right\}_{t=0}^{T}$ into the sequence $\left\{\tilde{k}_{t+1}\right\}_{t=0}^{T}$. By the Brouwer's fixed point theorem, there is a sequence $\left\{k_{t+1}^{*}\right\}_{t=0}^{T} \in \mathcal{S}_{T}$, which is a fixed point of this mapping. 


\section{Step 3. Useful claims.}

For the obtained fixed point $\left\{k_{t+1}^{*}\right\}_{t=0}^{T}$, let $\left\{r_{t+1}^{*}\right\}_{t=0}^{T}$ and $\left\{w_{t+1}^{*}\right\}_{t=0}^{T}$ be given by (13). Let also $\left\{c_{t}^{* * \tau}, s_{t}^{* * \tau}\right\}_{t=\tau}^{T+1}$ be a $T$-horizon date- $\tau$ consumer optimum starting from $k_{\tau}^{*}$ at given $\left\{r_{t+1}^{*}\right\}_{t=\tau}^{T}$ and $\left\{w_{t+1}^{*}\right\}_{t=\tau}^{T}$. Denote the date- $\tau$ consumption on this $T$-horizon date- $\tau$ consumer optimum by $c_{\tau}^{*}=c_{\tau}^{* * \tau}$. By C.1, for all $\tau=0, \ldots, T$, we have

$$
c_{\tau}^{*}=\frac{f\left(k_{\tau}^{*}\right)+\frac{w_{\tau+1}^{*}}{1+r_{\tau+1}^{*}}+\ldots+\frac{w_{T+1}^{*}}{\left(1+r_{\tau+1}^{*}\right) \cdots\left(1+r_{T+1}^{*}\right)}}{1+(\beta \delta)^{\frac{1}{\rho}}\left(1+r_{\tau+1}^{*}\right)^{\frac{1-\rho}{\rho}}+\ldots+\left(\beta \delta^{T+1-\tau}\right)^{\frac{1}{\rho}}\left(1+r_{\tau+1}^{*}\right)^{\frac{1-\rho}{\rho}} \cdots\left(1+r_{T+1}^{*}\right)^{\frac{1-\rho}{\rho}}} .
$$

The following claim establishes a useful property of the sequence $\left\{c_{t}^{*}\right\}_{t=0}^{T}$.

Claim C.2. For $t=0, \ldots, T-1$,

$$
c_{t+1}^{*} \geq c_{t}^{*}\left(\beta \delta\left(1+r_{t+1}^{*}\right)\right)^{\frac{1}{\rho}} \frac{1+\Delta_{t}^{T}}{1+\beta^{\frac{1}{\rho}} \Delta_{t}^{T}} \quad\left(=\text { if } k_{t+1}^{*}>\underline{k}_{t+1}\right),
$$

where

$$
\Delta_{t}^{T}=\delta^{\frac{1}{\rho}}\left(1+r_{t+2}^{*}\right)^{\frac{1-\rho}{\rho}}+\ldots+\delta^{\frac{T+1-t}{\rho}}\left(1+r_{t+2}^{*}\right)^{\frac{1-\rho}{\rho}} \cdots\left(1+r_{T+1}^{*}\right)^{\frac{1-\rho}{\rho}}
$$

Proof. Note that for $t=0, \ldots, T-2$, we have (cf. equation (19))

$$
\Delta_{t}^{T}=\delta^{\frac{1}{\rho}}\left(1+r_{t+2}^{*}\right)^{\frac{1-\rho}{\rho}}\left(1+\Delta_{t+1}^{T}\right)
$$

Then (C.6) can be rewritten as

$$
\begin{aligned}
c_{t}^{*}\left(1+(\beta \delta)^{\frac{1}{\rho}}\left(1+r_{t+1}^{*}\right)^{\frac{1-\rho}{\rho}}\left(1+\Delta_{t}^{T}\right)\right) & \\
& =f\left(k_{t}^{*}\right)+\frac{w_{t+1}^{*}}{1+r_{t+1}^{*}}+\ldots+\frac{w_{T+1}^{*}}{\left(1+r_{t+1}^{*}\right) \cdots\left(1+r_{T+1}^{*}\right)} .
\end{aligned}
$$

By construction of $k_{\tau+1}^{*}$, we have

$$
c_{\tau}^{*}+k_{\tau+1}^{*} \geq f\left(k_{\tau}^{*}\right) \quad\left(=\text { if } k_{\tau+1}^{*}>\underline{k}_{\tau+1}\right)
$$

and hence it follows from (C.9) for $t=\tau$ that

$$
c_{\tau}^{*}(\beta \delta)^{\frac{1}{\rho}}\left(1+r_{\tau+1}^{*}\right)^{\frac{1-\rho}{\rho}}\left(1+\Delta_{\tau}^{T}\right) \leq k_{\tau+1}^{*}+\frac{w_{t+1}^{*}}{1+r_{t+1}^{*}}+\ldots+\frac{w_{T+1}^{*}}{\left(1+r_{t+1}^{*}\right) \cdots\left(1+r_{T+1}^{*}\right)},
$$

(with equality when $k_{\tau+1}^{*}>\underline{k}_{\tau+1}$ ).

Now consider the value $c_{\tau+1}^{*}\left(1+\beta^{\frac{1}{\rho}} \Delta_{\tau}^{T}\right)$. Using (C.8), (C.9) for $t=\tau+1$ and (C.10), we 
get

$$
\begin{aligned}
c_{\tau+1}^{*}\left(1+\beta^{\frac{1}{\rho}} \Delta_{\tau}^{T}\right) & =c_{\tau+1}^{*}\left(1+(\beta \delta)^{\frac{1}{\rho}}\left(1+r_{\tau+2}^{*}\right)^{\frac{1-\rho}{\rho}}\left(1+\Delta_{\tau+1}^{T}\right)\right) \\
& =f\left(k_{\tau+1}^{*}\right)+\frac{w_{\tau+2}^{*}}{1+r_{\tau+2}^{*}}+\ldots+\frac{w_{T+1}^{*}}{\left(1+r_{\tau+2}^{*}\right) \cdots\left(1+r_{T+1}^{*}\right)} \\
= & \left(1+r_{\tau+1}^{*}\right) k_{\tau+1}^{*}+w_{\tau+1}^{*}+\frac{w_{\tau+2}^{*}}{1+r_{\tau+2}^{*}}+\ldots+\frac{w_{T+1}^{*}}{\left(1+r_{\tau+2}^{*}\right) \cdots\left(1+r_{T+1}^{*}\right)} \\
=\left(1+r_{\tau+1}^{*}\right)\left(k_{\tau+1}^{*}+\frac{w_{\tau+1}^{*}}{1+r_{\tau+1}^{*}}+\ldots+\frac{w_{T+1}^{*}}{\left(1+r_{\tau+1}^{*}\right) \cdots\left(1+r_{T+1}^{*}\right)}\right) & \geq c_{\tau}^{*}(\beta \delta)^{\frac{1}{\rho}}\left(1+r_{\tau+1}^{*}\right)^{\frac{1}{\rho}}\left(1+\Delta_{\tau}^{T}\right),
\end{aligned}
$$

(again, with equality when $k_{\tau+1}^{*}>\underline{k}_{\tau+1}$ ).

Therefore, the consumption levels $c_{\tau+1}^{*}$ and $c_{\tau}^{*}$ are linked as follows:

$$
c_{\tau+1}^{*} \geq c_{\tau}^{*}\left(\beta \delta\left(1+r_{\tau+1}^{*}\right)\right)^{\frac{1}{\rho}} \frac{1+\Delta_{\tau}^{T}}{1+\beta^{\frac{1}{\rho}} \Delta_{\tau}^{T}}, \quad\left(=\text { if } \quad k_{\tau+1}^{*}>\underline{k}_{\tau+1}\right),
$$

and this holds true for all $\tau=0, \ldots, T-1$.

Claim C.2 allows us to prove that the lower bound in $\mathcal{S}_{T}$ is never binding for our fixed point.

Claim C.3. For all $t=0, \ldots, T$, we have $k_{t+1}^{*}>\underline{k}_{t+1}$.

Proof. First, let us prove that $k_{t+1}^{*}>\underline{k}_{t+1}$ for all $t<T$. Suppose the opposite, i.e., that $k_{\tau+1}^{*}=\underline{k}_{\tau+1}$ for some $\tau=0, \ldots, T-1$. Then, by construction of $k_{\tau+1}^{*}$,

$$
c_{\tau}^{*} \geq f\left(k_{\tau}^{*}\right)-k_{\tau+1}^{*} \geq f\left(\underline{k}_{\tau}\right)-k_{\tau+1}^{*}=f\left(\underline{k}_{\tau}\right)-\underline{k}_{\tau+1}=\underline{c}_{\tau} .
$$

Let us show that in this case $k_{\tau+2}^{*}=\underline{k}_{\tau+2}$. Indeed, suppose the opposite, i.e., $k_{\tau+2}^{*}>\underline{k}_{\tau+2}$. Then, by construction of $k_{\tau+2}^{*}$,

$$
c_{\tau+1}^{*}=f\left(k_{\tau+1}^{*}\right)-k_{\tau+2}^{*}=f\left(\underline{k}_{\tau+1}\right)-k_{\tau+2}^{*}<f\left(\underline{k}_{\tau+1}\right)-\underline{k}_{\tau+2}=\underline{c}_{\tau+1} .
$$

At the same time, since $1+\Delta_{\tau}^{T}>1+\beta^{\frac{1}{\rho}} \Delta_{\tau}^{T}$, it follows from (C.7), (C.11) and (C.4) that

$$
c_{\tau+1}^{*} \geq c_{\tau}^{*}\left(\beta \delta\left(1+r_{\tau+1}^{*}\right)\right)^{\frac{1}{\rho}} \frac{1+\Delta_{\tau}^{T}}{1+\beta^{\frac{1}{\rho}} \Delta_{\tau}^{T}}>\left(\beta \delta\left(1+r_{\tau+1}^{*}\right)\right)^{\frac{1}{\rho}} c_{\tau}^{*} \geq\left(\beta \delta\left(\underline{r}_{\tau+1}\right)\right)^{\frac{1}{\rho}} \underline{c}_{\tau}=\underline{c}_{\tau+1} .
$$

This contradiction shows that $k_{\tau+2}^{*}=\underline{k}_{\tau+2}$. Similarly to (C.11), it also follows that

$$
c_{\tau+1}^{*} \geq f\left(k_{\tau+1}^{*}\right)-k_{\tau+2}^{*}=f\left(\underline{k}_{\tau+1}\right)-\underline{k}_{\tau+2}=\underline{c}_{\tau+1} .
$$

Repeating the argument, we obtain that if $k_{\tau+1}^{*}=\underline{k}_{\tau+1}$, then $k_{t+1}^{*}=\underline{k}_{t+1}$ for all $t=$ $\tau+1, \ldots, T$, and hence the sequence $\left\{k_{t+1}^{*}\right\}_{t=\tau}^{T}$ coincides with the sequence $\left\{\underline{k}_{t+1}\right\}_{t=\tau}^{T}$. However, in this case for all $t=\tau, \ldots, T$, we have $1+r_{t+1}^{*}=1+\underline{r}_{t+1}$ and $w_{t+1}^{*}=\underline{w}_{t+1}$, and it follows 
from (C.3) and (C.6) that

$$
\begin{aligned}
\underline{c}_{\tau+1}= & \frac{f\left(\underline{k}_{\tau+1}\right)+\frac{\underline{w}_{\tau+2}}{1+\underline{r}_{\tau+2}}+\ldots+\frac{\underline{w}_{T+1}}{\left(1+\underline{r}_{\tau+2}\right) \cdots\left(1+\underline{r}_{T+1}\right)}}{1+(\beta \delta)^{\frac{1}{\rho}}\left(1+\underline{r}_{\tau+2}\right)^{\frac{1-\rho}{\rho}}+\ldots+(\beta \delta)^{\frac{T+1-\tau}{\rho}}\left(1+\underline{r}_{\tau+2}\right)^{\frac{1-\rho}{\rho}} \cdots\left(1+\underline{r}_{T+1}\right)^{\frac{1-\rho}{\rho}}} \\
& =\frac{f\left(k_{\tau+1}^{*}\right)+\frac{w_{\tau+2}^{*}}{1+r_{\tau+2}^{*}}+\ldots+\frac{w_{T+1}^{*}}{\left(1+r_{\tau+2}^{*}\right)^{*}\left(1+r_{T+1}^{*}\right)}}{1+(\beta \delta)^{\frac{1}{\rho}}\left(1+r_{\tau+2}^{*}\right)^{\frac{1-\rho}{\rho}}+\ldots+(\beta \delta)^{\frac{T+1-\tau}{\rho}}\left(1+r_{\tau+2}^{*}\right)^{\frac{1-\rho}{\rho}} \cdots\left(1+r_{T+1}^{*}\right)^{\frac{1-\rho}{\rho}}} \\
& >\frac{f\left(k_{\tau+1}^{*}\right)+\frac{w_{\tau+2}^{*}}{1+r_{\tau+2}^{*}}+\ldots+\frac{w_{T+1}^{*}}{\left(1+r_{\tau+2}^{*}\right)^{* \cdots}\left(1+r_{T+1}^{*}\right)}}{1+(\beta \delta)^{\frac{1}{\rho}}\left(1+r_{\tau+2}^{*}\right)^{\frac{1-\rho}{\rho}}+\ldots+\left(\beta \delta^{T+1-\tau}\right)^{\frac{1}{\rho}}\left(1+r_{\tau+2}^{*}\right)^{\frac{1-\rho}{\rho}} \cdots\left(1+r_{T+1}^{*}\right)^{\frac{1-\rho}{\rho}}}=c_{\tau+1}^{*},
\end{aligned}
$$

because in the denominator $\beta<1$. This contradiction to (C.12) shows that $k_{t+1}^{*}>\underline{k}_{t+1}$ for all $t=0, \ldots, T-1$.

Second, let us prove that $k_{T+1}^{*}>\underline{k}_{T+1}$. Suppose the opposite, i.e., that $k_{T+1}^{*}=\underline{k}_{T+1}$, and hence $1+r_{T+1}^{*}=1+\underline{r}_{T+1}$ and $w_{T+1}^{*}=\underline{w}_{T+1}$. Also, by construction of $k_{T+1}^{*}$,

$$
c_{T}^{*} \geq f\left(k_{T}^{*}\right)-k_{T+1}^{*} \geq f\left(\underline{k}_{T}\right)-k_{T+1}^{*}=f\left(\underline{k}_{T}\right)-\underline{k}_{T+1}=\underline{c}_{T} .
$$

Consider now the $T$-horizon date- $T$ consumer optimum starting from $k_{T}^{*}$ at given $1+r_{T+1}^{*}$ and $w_{T+1}^{*}$. It follows from the first-order condition, (C.13) and (C.4) that

$$
c_{T+1}^{* * T}=\left(\beta \delta\left(1+r_{T+1}^{*}\right)\right)^{\frac{1}{\rho}} c_{T}^{*}=\left(\beta \delta\left(1+\underline{r}_{T+1}\right)\right)^{\frac{1}{\rho}} c_{T}^{*}>\left(\beta \delta\left(1+\underline{r}_{T+1}\right)\right)^{\frac{1}{\rho}} \underline{c}_{T}=\underline{c}_{T+1} .
$$

At the same time, it follows from the budget constraint, (C.13) and (C.5) that

$$
\begin{aligned}
c_{T+1}^{* * T}=\left(1+r_{T+1}^{*}\right)\left(f\left(k_{T}^{*}\right)-c_{T}^{*}\right)+ & w_{T+1}^{*} \leq\left(1+r_{T+1}^{*}\right) k_{T+1}^{*}+w_{T+1}^{*}=f\left(k_{T+1}^{*}\right) \\
= & f\left(\underline{k}_{T+1}\right)=\left(1+\underline{r}_{T+1}\right)\left(f\left(\underline{k}_{T}\right)-\underline{c}_{T}\right)+\underline{w}_{T+1}=\underline{c}_{T+1} .
\end{aligned}
$$

This contradiction shows that $k_{T+1}^{*}>\underline{k}_{T+1}$, which completes the proof of the claim.

\section{Step 4. Ensuring existence.}

Claim C.3 shows that for all $\tau=0, \ldots, T$, our obtained fixed point satisfies $k_{\tau+1}^{*}>\underline{k}_{\tau+1}$. Let for all $\tau=0, \ldots, T, s_{\tau}^{*}=k_{\tau+1}^{*}$. Then we have $s_{\tau}^{*}=f\left(k_{\tau}^{*}\right)-c_{\tau}^{*}$, and hence by construction consumption $c_{\tau}^{*}$ and savings $s_{\tau}^{*}$ are precisely the first elements of the $T$-horizon date- $\tau$ consumer optimum starting from $k_{\tau}^{*}$ at given $\left\{r_{t+1}^{*}\right\}_{t=\tau}^{T}$ and $\left\{w_{t+1}^{*}\right\}_{t=\tau}^{T}$.

It follows that the obtained fixed point determines the $T$-horizon SEP under perfect foresight starting from $s_{-1}^{*}=k_{0}^{*}$. Formally, the sequence $\left\{c_{t}^{*}, s_{t}^{*}, k_{t+1}^{*}, r_{t+1}^{*}, w_{t+1}^{*}\right\}_{t=0}^{T}$, where $\left\{k_{t+1}^{*}\right\}_{t=0}^{T}$ is the fixed point of the described mapping $\mathcal{S}_{T} \rightarrow \mathcal{S}_{T}$, and for all $\tau=0, \ldots, T$,

$$
\begin{gathered}
c_{\tau}^{*}=f\left(k_{\tau}^{*}\right)-k_{\tau+1}^{*}, \quad s_{\tau}^{*}=k_{\tau+1}^{*}, \\
1+r_{\tau}^{*}=f^{\prime}\left(k_{\tau}^{*}\right), \quad w_{\tau}^{*}=f\left(k_{\tau}^{*}\right)-f^{\prime}\left(k_{\tau}^{*}\right) k_{\tau}^{*},
\end{gathered}
$$

satisfies Definition 9 and hence is a $T$-horizon SEP under perfect foresight. 


\section{C.3 Proof of Lemma 2.2}

It follows from Claim C.3 that the sequence of capital stocks on a $T$-horizon SEP under perfect foresight is bounded from below by the sequence of capital stocks $\left\{\underline{k}_{t+1}(T)\right\}_{t=0}^{T}$. It remains to show that it is also bounded from above.

\section{Step 1. Upper bounds for capital.}

Let $\left\{\bar{c}_{t}(T), \bar{k}_{t+1}(T)\right\}_{t=0}^{T+1}$ be a solution to problem (15). Let also $\bar{s}_{-1}=\bar{k}_{0}$, and for $t=$ $0, \ldots, T+1$

$$
\bar{s}_{t}(T)=\bar{k}_{t+1}(T), \quad 1+\bar{r}_{t}(T)=f^{\prime}\left(\bar{k}_{t}(T)\right), \quad \bar{w}_{t}(T)=f\left(\bar{k}_{t}(T)\right)-f^{\prime}\left(\bar{k}_{t}(T)\right) \bar{k}_{t}(T) .
$$

Then the sequence $\left\{\bar{c}_{t}(T), \bar{s}_{t}(T), \bar{k}_{t+1}(T), \bar{r}_{t}(T), \bar{w}_{t}(T)\right\}_{t=0}^{T+1}$ is a $T$-horizon equilibrium path starting from $\bar{s}_{-1}$ in the standard Ramsey model with a discount factor $\delta$. Again, in what follows we shall omit the notation " $(T)$ ", as this does not lead to confusion.

Similarly to (C.4) and (C.5), it is easily checked that $\left\{\bar{c}_{t}\right\}_{t=0}^{T+1}$ satisfies

$$
\bar{c}_{t+1}=\left(\delta\left(1+\bar{r}_{t+1}\right)\right)^{\frac{1}{\rho}} \bar{c}_{t}, \quad t=0, \ldots, T,
$$

and for all $\tau=0, \ldots, T$,

$$
\begin{aligned}
\bar{c}_{\tau}+\frac{\bar{c}_{\tau+1}}{1+\bar{r}_{\tau+1}}+\ldots+\frac{\bar{c}_{T+1}}{\left(1+\bar{r}_{\tau+1}\right) \cdots\left(1+\bar{r}_{T+1}\right)} & \\
= & f\left(\bar{k}_{\tau}\right)+\frac{\bar{w}_{\tau+1}}{1+\bar{r}_{\tau+1}}+\ldots+\frac{\bar{w}_{T+1}}{\left(1+\bar{r}_{\tau+1}\right) \cdots\left(1+\bar{r}_{T+1}\right)}
\end{aligned}
$$

\section{Step 2. Upper and lower bounds for the consumption growth rate.}

Let $\left\{c_{t}^{*}, s_{t}^{*}, k_{t+1}^{*}, r_{t+1}^{*}, w_{t+1}^{*}\right\}_{t=0}^{T}$ be a $T$-horizon SEP under perfect foresight starting from $s_{-1}^{*}=k_{0}^{*}$. We already know that date- $\tau$ consumption on a $T$-horizon SEP under perfect foresight is given by (C.6). The following claim provides upper and lower bounds for the consumption growth rate on a $T$-horizon SEP under perfect foresight.

Claim C.4. The consumption growth rate on a T-horizon SEP under perfect foresight satisfies

$$
\left(\beta \delta\left(1+r_{t+1}^{*}\right)\right)^{\frac{1}{\rho}}<\frac{c_{t+1}^{*}}{c_{t}^{*}}<\left(\delta\left(1+r_{t+1}^{*}\right)\right)^{\frac{1}{\rho}} .
$$

Proof. By Claim C.3, on a T-horizon SEP under perfect foresight we have $k_{t+1}^{*}>\underline{k}_{t+1}$, and it follows from Claim C.2 that the consumption levels at two adjacent dates are linked via the following "first-order conditions" (cf. equation (20)): for all $t=0, \ldots, T-1$,

$$
c_{t+1}^{*}=c_{t}^{*}\left(\beta \delta\left(1+r_{t+1}^{*}\right)\right)^{\frac{1}{\rho}} \frac{1+\Delta_{t}^{T}}{1+\beta^{\frac{1}{\rho}} \Delta_{t}^{T}} .
$$


Since evidently $1+\Delta_{t}^{T}>1+\beta^{\frac{1}{\rho}} \Delta_{t}^{T}>\beta^{\frac{1}{\rho}}+\beta^{\frac{1}{\rho}} \Delta_{t}^{T}$, we have

$$
\frac{c_{t+1}^{*}}{c_{t}^{*}}=\left(\beta \delta\left(1+r_{t+1}^{*}\right)\right)^{\frac{1}{\rho}} \frac{1+\Delta_{t}^{T}}{1+\beta^{\frac{1}{\rho}} \Delta_{t}^{T}}>\left(\beta \delta\left(1+r_{t+1}^{*}\right)\right)^{\frac{1}{\rho}}
$$

and

$$
\frac{c_{t+1}^{*}}{c_{t}^{*}}=\left(\delta\left(1+r_{t+1}^{*}\right)\right)^{\frac{1}{\rho}} \frac{\beta^{\frac{1}{\rho}}+\beta^{\frac{1}{\rho}} \Delta_{t}^{T}}{1+\beta^{\frac{1}{\rho}} \Delta_{t}^{T}}<\left(\delta\left(1+r_{t+1}^{*}\right)\right)^{\frac{1}{\rho}},
$$

and hence (C.16) holds.

\section{Step 3. Proof of the lemma.}

Now we can show that the sequence of capital stocks on a $T$-horizon SEP under perfect foresight is bounded from above by the sequence of capital stocks $\left\{\bar{k}_{t+1}(T)\right\}_{t=0}^{T}$.

Claim C.5. For all $t=0, \ldots, T$, we have $k_{t+1}^{*}<\bar{k}_{t+1}$.

Proof. Suppose the opposite, i.e., that while $k_{0}^{*}=\bar{k}_{0}$ and $k_{\tau}^{*}<\bar{k}_{\tau}\left(\right.$ if $\tau>0$ ), we have $k_{\tau+1}^{*} \geq \bar{k}_{\tau+1}$ for some $\tau=0, \ldots, T-1$. Then

$$
c_{\tau}^{*}=f\left(k_{\tau}^{*}\right)-k_{\tau+1}^{*} \leq f\left(\bar{k}_{\tau}\right)-k_{\tau+1}^{*} \leq f\left(\bar{k}_{\tau}\right)-\bar{k}_{\tau+1}=\bar{c}_{\tau} .
$$

Let us show that in this case $k_{\tau+2}^{*} \geq \bar{k}_{\tau+2}$. Indeed, suppose the opposite, i.e., $k_{\tau+2}^{*}<\bar{k}_{\tau+2}$. Then

$$
c_{\tau+1}^{*}=f\left(k_{\tau+1}^{*}\right)-k_{\tau+2}^{*} \geq f\left(\bar{k}_{\tau+1}\right)-k_{\tau+2}^{*}>f\left(\bar{k}_{\tau+1}\right)-\bar{k}_{\tau+2}=\bar{c}_{\tau+1} .
$$

At the same time, it follows from (C.16), (C.17) and (C.14) that

$$
c_{\tau+1}^{*}<\left(\delta\left(1+r_{t+1}^{*}\right)\right)^{\frac{1}{\rho}} c_{\tau}^{*} \leq\left(\delta\left(1+\bar{r}_{t+1}\right)\right)^{\frac{1}{\rho}} c_{\tau}^{*} \leq\left(\delta\left(1+\bar{r}_{t+1}\right)\right)^{\frac{1}{\rho}} \bar{c}_{\tau}=\bar{c}_{\tau+1} .
$$

This contradiction shows that $k_{\tau+2}^{*} \geq \bar{k}_{\tau+2}$.

Repeating the argument, we obtain that if $k_{\tau+1}^{*} \geq \bar{k}_{\tau+1}$, then $k_{t+1}^{*} \geq \bar{k}_{t+1}$ for all $t=$ $\tau+1, \ldots, T$. Moreover, it then follows from (C.16) that $c_{t}^{*} \leq \bar{c}_{t}$ for all $t=\tau, \ldots, T$.

Consider now the $T$-horizon date- $T$ consumer optimum starting from $k_{T}^{*}$ at given $1+r_{T+1}^{*}$ and $w_{T+1}^{*}$. Since $\beta<1$, it follows from the first-order condition and (C.14) that

$$
\begin{aligned}
c_{T+1}^{* * T}=\left(\beta \delta\left(1+r_{T+1}^{*}\right)\right)^{\frac{1}{\rho}} c_{T}^{*}<(\delta(1+ & \left.\left.r_{T+1}^{*}\right)\right)^{\frac{1}{\rho}} c_{T}^{*} \\
& \leq\left(\delta\left(1+\bar{r}_{t+1}\right)\right)^{\frac{1}{\rho}} c_{T}^{*} \leq\left(\delta\left(1+\bar{r}_{t+1}\right)\right)^{\frac{1}{\rho}} \bar{c}_{T}=\bar{c}_{T+1} .
\end{aligned}
$$

However, it follows from the budget constraint and (C.15) that

$$
\begin{aligned}
c_{T+1}^{* * T}=\left(1+r_{T+1}^{*}\right)\left(f\left(k_{T}^{*}\right)-c_{T}^{*}\right) & +w_{T+1}^{*}=\left(1+r_{T+1}^{*}\right) k_{T+1}^{*}+w_{T+1}^{*}=f\left(k_{T+1}^{*}\right) \\
& \geq f\left(\bar{k}_{T+1}\right)=\left(1+\bar{r}_{T+1}\right)\left(f\left(\bar{k}_{T}\right)-\bar{c}_{T}\right)+\bar{w}_{T+1}=\bar{c}_{T+1},
\end{aligned}
$$

and this contradiction shows that indeed $k_{t+1}^{*}<\bar{k}_{t+1}$ for all $t=0, \ldots, T$. 


\section{C.4 Proof of Lemma 2.3}

\section{Step 1. Upper and lower bounds for capital.}

Let $\left\{\underline{c}_{t}, \underline{k}_{t+1}\right\}_{t=0}^{\infty}$ be the $\beta \delta$-optimal path, i.e., the optimal path starting from $\underline{k}_{0}=k_{0}^{*}$ in the standard Ramsey model with a discount factor $\beta \delta$. It is clear that

$$
\underline{c}_{t}=\lim _{T \rightarrow \infty} \underline{c}_{t}(T), \quad \underline{k}_{t+1}=\lim _{T \rightarrow \infty} \underline{k}_{t+1}(T) .
$$

Similarly, let $\left\{\bar{c}_{t}, \bar{k}_{t+1}\right\}_{t=0}^{\infty}$ be the $\delta$-optimal path, i.e., the optimal path starting from $\bar{k}_{0}=k_{0}^{*}$ in the standard Ramsey model with a discount factor $\delta$. As above, it is clear that

$$
\bar{c}_{t}=\lim _{T \rightarrow \infty} \bar{c}_{t}(T), \quad \bar{k}_{t+1}=\lim _{T \rightarrow \infty} \bar{k}_{t+1}(T) .
$$

\section{Step 2. A closed-form expression for consumption.}

Note that by construction of the sequence $\left\{c_{t}^{*}, s_{t}^{*}, k_{t+1}^{*}, r_{t+1}^{*}, w_{t+1}^{*}\right\}_{t=0}^{\infty}$,

$$
\begin{gathered}
c_{t}^{*}=\lim _{T \rightarrow \infty} c_{t}^{*}(T), \quad s_{t}^{*}=\lim _{T \rightarrow \infty} s_{t}^{*}(T), \\
k_{t+1}^{*}=\lim _{T \rightarrow \infty} k_{t+1}^{*}(T), \quad r_{t+1}^{*}=\lim _{T \rightarrow \infty} r_{t+1}^{*}(T), \quad w_{t+1}^{*}=\lim _{T \rightarrow \infty} w_{t+1}^{*}(T) .
\end{gathered}
$$

Taking the limit $T \rightarrow \infty$ in (16), we obtain that for all $t=0,1, \ldots$,

$$
\underline{k}_{t+1} \leq k_{t+1}^{*} \leq \bar{k}_{t+1}
$$

It immediately follows from (17) and (C.18) that for all $t=0,1, \ldots$,

$$
\underline{s}_{t} \leq s_{t}^{*} \leq \bar{s}_{t}, \quad \underline{w}_{t} \leq w_{t}^{*} \leq \bar{w}_{t}, \quad \bar{r}_{t} \leq r_{t}^{*} \leq \underline{r}_{t} .
$$

Note also that by (17) and (C.18),

$$
c_{0}^{*}=f\left(k_{0}^{*}\right)-k_{1}^{*}=f\left(\bar{k}_{0}\right)-k_{1}^{*} \geq f\left(\bar{k}_{0}\right)-\bar{k}_{1}=\bar{c}_{0},
$$

and taking the limit $T \rightarrow \infty$ in (C.16), we get

$$
\frac{c_{t+1}^{*}}{c_{t}^{*}}=\lim _{T \rightarrow \infty} \frac{c_{t+1}^{*}(T)}{c_{t}^{*}(T)} \geq\left(\beta \delta\left(1+r_{t+1}^{*}\right)\right)^{\frac{1}{\rho}} \geq\left(\beta \delta\left(1+\bar{r}_{t+1}\right)\right)^{\frac{1}{\rho}},
$$

so that $c_{t}^{*}>0$ for all $t=0,1, \ldots$

Let us show that the consumption level $c_{t}^{*}$ is given by a limit of (C.6) as $T \rightarrow \infty$.

Claim C.6. For all $\tau=0,1, \ldots$, we have

$$
c_{\tau}^{*}=\frac{f\left(k_{\tau}^{*}\right)+\frac{w_{\tau+1}^{*}}{1+r_{\tau+1}^{*}}+\ldots+\frac{w_{\tau+t}^{*}}{\left(1+r_{\tau+1}^{*}\right)^{\cdots}\left(1+r_{\tau+t}^{*}\right)}+\ldots}{1+(\beta \delta)^{\frac{1}{\rho}}\left(1+r_{\tau+1}^{*}\right)^{\frac{1-\rho}{\rho}}+\ldots+\left(\beta \delta^{t}\right)^{\frac{1}{\rho}}\left(1+r_{\tau+1}^{*}\right)^{\frac{1-\rho}{\rho}} \cdots\left(1+r_{\tau+t}^{*}\right)^{\frac{1-\rho}{\rho}}+\ldots} .
$$


Proof. Note that (C.6) can be written as

$$
\begin{aligned}
c_{\tau}^{*}(T)\left(1+(\beta \delta)^{\frac{1}{\rho}}\right. & \left.\left(1+r_{\tau+1}^{*}(T)\right)^{\frac{1-\rho}{\rho}}+\ldots+\left(\beta \delta^{T+1-\tau}\right)^{\frac{1}{\rho}}\left(1+r_{\tau+1}^{*}(T)\right)^{\frac{1-\rho}{\rho}} \cdots\left(1+r_{T+1}^{*}(T)\right)^{\frac{1-\rho}{\rho}}\right) \\
& =f\left(k_{\tau}^{*}(T)\right)+\frac{w_{\tau+1}^{*}(T)}{1+r_{\tau+1}^{*}(T)}+\ldots+\frac{w_{T+1}^{*}(T)}{\left(1+r_{\tau+1}^{*}(T)\right) \cdots\left(1+r_{T+1}^{*}(T)\right)} \cdot \quad(\text { C. } 20)
\end{aligned}
$$

By (16), we have

$$
\begin{aligned}
f\left(k_{\tau}^{*}(T)\right)+\frac{w_{\tau+1}^{*}(T)}{1+r_{\tau+1}^{*}(T)} & +\ldots+\frac{w_{T+1}^{*}(T)}{\left(1+r_{\tau+1}^{*}(T)\right) \cdots\left(1+r_{T+1}^{*}(T)\right)} \\
& \leq f\left(\bar{k}_{\tau}(T)\right)+\frac{\bar{w}_{\tau+1}(T)}{1+\bar{r}_{\tau+1}(T)}+\ldots+\frac{\bar{w}_{T+1}(T)}{\left(1+\bar{r}_{\tau+1}(T)\right) \cdots\left(1+\bar{r}_{T+1}(T)\right)}
\end{aligned}
$$

and by the properties of the $\delta$-optimal path, there exists a finite limit of the right hand side in (C.20) as $T \rightarrow \infty$ :

$$
\begin{aligned}
& \lim _{T \rightarrow \infty}\left\{f\left(k_{\tau}^{*}(T)\right)+\right.\left.\frac{w_{\tau+1}^{*}(T)}{1+r_{\tau+1}^{*}(T)}+\ldots+\frac{w_{T+1}^{*}(T)}{\left(1+r_{\tau+1}^{*}(T)\right) \cdots\left(1+r_{T+1}^{*}(T)\right)}\right\} \\
&=f\left(k_{\tau}^{*}\right)+\frac{w_{\tau+1}^{*}}{1+r_{\tau+1}^{*}}+\ldots+\frac{w_{\tau+t}^{*}}{\left(1+r_{\tau+1}^{*}\right) \cdots\left(1+r_{\tau+t}^{*}\right)}+\ldots \\
& \quad \leq f\left(\bar{k}_{\tau}\right)+\frac{\bar{w}_{\tau+1}}{1+\bar{r}_{\tau+1}}+\ldots+\frac{\bar{w}_{\tau+t}}{\left(1+\bar{r}_{\tau+1}\right) \cdots\left(1+\bar{r}_{\tau+t}\right)}+\ldots
\end{aligned}
$$

It then follows from (C.20) that there exists a finite limit of its left hand side as $T \rightarrow \infty$, and hence the series

$$
\begin{array}{r}
\lim _{T \rightarrow \infty}\left\{1+(\beta \delta)^{\frac{1}{\rho}}\left(1+r_{\tau+1}^{*}(T)\right)^{\frac{1-\rho}{\rho}}+\ldots+\left(\beta \delta^{T+1-\tau}\right)^{\frac{1}{\rho}}\left(1+r_{\tau+1}^{*}(T)\right)^{\frac{1-\rho}{\rho}} \cdots\left(1+r_{T+1}^{*}(T)\right)^{\frac{1-\rho}{\rho}}\right\} \\
=1+(\beta \delta)^{\frac{1}{\rho}}\left(1+r_{\tau+1}^{*}\right)^{\frac{1-\rho}{\rho}}+\ldots+\left(\beta \delta^{t}\right)^{\frac{1}{\rho}}\left(1+r_{\tau+1}^{*}\right)^{\frac{1-\rho}{\rho}} \cdots\left(1+r_{\tau+t}^{*}\right)^{\frac{1-\rho}{\rho}}+\ldots
\end{array}
$$

converges.

Therefore, taking the limit $T \rightarrow \infty$ in (C.20), we obtain (C.19).

\section{Step 3. Infinite horizon date- $\tau$ consumer optimum.}

For each $\tau=0,1, \ldots$, consider the sequence $\left\{c_{t}^{* * \tau}\right\}_{t=\tau}^{\infty}$, which is defined as follows:

$$
\begin{aligned}
& c_{\tau}^{* * \tau}=c_{\tau}^{*}, \quad c_{\tau+1}^{* * \tau}=\left(\beta \delta\left(1+r_{\tau+1}^{*}\right)\right)^{\frac{1}{\rho}} c_{\tau}^{* * \tau}, \\
& c_{t+1}^{* * \tau}=\left(\delta\left(1+r_{t+1}^{*}\right)\right)^{\frac{1}{\rho}} c_{t}^{* * \tau}, \quad t=\tau+1, \tau+2, \ldots
\end{aligned}
$$

The following claim ensures that $\left\{c_{t}^{* * \tau}\right\}_{t=\tau}^{\infty}$ is the sequence of consumptions on the date- $\tau$ consumer optimum starting from $k_{\tau}^{*}$ at given $\left\{r_{t+1}^{*}\right\}_{t=\tau}^{\infty}$ and $\left\{w_{t+1}^{*}\right\}_{t=\tau}^{\infty}$.

Claim C.7. The sequence $\left\{c_{t}^{* * \tau}\right\}_{t=\tau}^{\infty}$ is the sequence of consumptions on the date- $\tau$ consumer optimum starting from $k_{\tau}^{*}$ at given $\left\{r_{t+1}^{*}\right\}_{t=\tau}^{\infty}$ and $\left\{w_{t+1}^{*}\right\}_{t=\tau}^{\infty}$. 
Proof. Let us show that the sequence $\left\{c_{t}^{* * \tau}\right\}_{t=\tau}^{\infty}$ is the solution to the following problem:

$$
\begin{array}{ll}
\max _{\left\{c_{t}, s_{t}\right\}} & u\left(c_{\tau}\right)+\beta \sum_{t=\tau+1}^{\infty} \delta^{t-\tau} u\left(c_{t}\right) \\
\text { s. t. } & c_{\tau}+\frac{c_{\tau+1}}{1+r_{\tau+1}^{*}}+\ldots+\frac{c_{\tau+t}}{\left(1+r_{\tau+1}^{*}\right) \cdots\left(1+r_{\tau+t}^{*}\right)}+\ldots \\
& \leq f\left(k_{\tau}^{*}\right)+\frac{w_{\tau+1}^{*}}{1+r_{\tau+1}^{*}}+\ldots+\frac{w_{\tau+t}^{*}}{\left(1+r_{\tau+1}^{*}\right) \cdots\left(1+r_{\tau+t}^{*}\right)}+\ldots, \\
& c_{t} \geq 0, \quad t=\tau, \tau+1, \ldots
\end{array}
$$

It follows from (C.21) that $\left\{c_{t}^{* * \tau}\right\}_{t=\tau}^{\infty}$ satisfies the first-order conditions for the solution to problem (C.22). Furthermore, by combining (C.21) and (C.19), it is easily seen that

$$
\begin{aligned}
c_{\tau}^{* * \tau}+ & \frac{c_{\tau+1}^{* * \tau}}{1+r_{\tau+1}^{*}}+\ldots+\frac{c_{\tau+t}^{* * \tau}}{\left(1+r_{\tau+1}^{*}\right) \cdots\left(1+r_{\tau+t}^{*}\right)}+\ldots \\
= & c_{\tau}^{*}\left(1+(\beta \delta)^{\frac{1}{\rho}}\left(1+r_{\tau+1}^{*}\right)^{\frac{1-\rho}{\rho}}+\ldots+\left(\beta \delta^{t}\right)^{\frac{1}{\rho}}\left(1+r_{\tau+1}^{*}\right)^{\frac{1-\rho}{\rho}} \cdots\left(1+r_{\tau+t}^{*}\right)^{\frac{1-\rho}{\rho}}+\ldots\right) \\
& =f\left(k_{\tau}^{*}\right)+\frac{w_{\tau+1}^{*}}{1+r_{\tau+1}^{*}}+\ldots+\frac{w_{\tau+t}^{*}}{\left(1+r_{\tau+1}^{*}\right) \cdots\left(1+r_{\tau+t}^{*}\right)}+\ldots,
\end{aligned}
$$

so that $\left\{c_{t}^{* * \tau}\right\}_{t=\tau}^{\infty}$ satisfies the budget constraint in problem (C.22).

Finally, consider the total utility on the consumption path $\left\{c_{t}^{* * \tau}\right\}_{t=\tau}^{\infty}$ :

$$
U_{\tau}=u\left(c_{\tau}^{* * \tau}\right)+\beta \delta u\left(c_{\tau+1}^{* * \tau}\right)+\ldots+\beta \delta^{t} u\left(c_{\tau+t}^{* * \tau}\right)+\ldots
$$

Using (C.21), we obtain that

$$
\begin{aligned}
& U_{\tau}=\frac{\left(c_{\tau}^{* * \tau}\right)^{1-\rho}}{1-\rho}+\beta \delta \frac{\left(c_{\tau+1}^{* * \tau}\right)^{1-\rho}}{1-\rho}+\ldots+\beta \delta^{t} \frac{\left(c_{\tau+t}^{* * \tau}\right)^{1-\rho}}{1-\rho}+\ldots \\
& =\frac{\left(c_{\tau}^{* * \tau}\right)^{1-\rho}}{1-\rho}\left(1+\beta \delta\left(\frac{c_{\tau+1}^{* * \tau}}{c_{\tau}^{* * \tau}}\right)^{1-\rho}+\ldots+\beta \delta^{t}\left(\frac{c_{\tau+t}^{* * \tau}}{c_{\tau}^{* * \tau}}\right)^{1-\rho}+\ldots\right) \\
& =\frac{\left(c_{\tau}^{* * \tau}\right)^{1-\rho}}{1-\rho}\left(1+\beta \delta\left(\beta \delta\left(1+r_{\tau+1}^{*}\right)\right)^{\frac{1-\rho}{\rho}}+\ldots+\beta \delta^{t}\left(\beta \delta^{t}\left(1+r_{\tau+1}^{*}\right) \cdots\left(1+r_{\tau+t}^{*}\right)\right)^{\frac{1-\rho}{\rho}}+\ldots\right) \\
& =\frac{1}{1-\rho} \frac{c_{\tau}^{* * \tau}}{\left(c_{\tau}^{* * \tau}\right)^{\rho}}\left(1+(\beta \delta)^{\frac{1}{\rho}}\left(1+r_{\tau+1}^{*}\right)^{\frac{1-\rho}{\rho}}+\ldots+\left(\beta \delta^{t}\right)^{\frac{1}{\rho}}\left(1+r_{\tau+1}^{*}\right)^{\frac{1-\rho}{\rho}} \ldots\left(1+r_{\tau+t}^{*}\right)^{\frac{1-\rho}{\rho}}+\ldots\right) \\
& =\frac{1}{1-\rho} \frac{1}{\left(c_{\tau}^{*}\right)^{\rho}}\left(f\left(k_{\tau}^{*}\right)+\frac{w_{\tau+1}^{*}}{1+r_{\tau+1}^{*}}+\ldots+\frac{w_{\tau+t}^{*}}{\left(1+r_{\tau+1}^{*}\right) \cdots\left(1+r_{\tau+t}^{*}\right)}+\ldots\right),
\end{aligned}
$$

where the last equality follows from (C.19). It is now clear that $-\infty<U_{\tau}<\infty$, which completes the proof of the claim.

\section{Step 4. Ensuring existence.}

Claim C.7 shows that the sequence $\left\{c_{t}^{* * \tau}\right\}_{t=\tau}^{\infty}$, determined by (C.21), is the sequence of consumptions on the date- $\tau$ consumer optimum starting from $k_{\tau}^{*}$ at given $\left\{r_{t+1}^{*}\right\}_{t=\tau}^{\infty}$ and $\left\{w_{t+1}^{*}\right\}_{t=\tau}^{\infty}$. 
Therefore, for each $\tau$, consumption $c_{\tau}^{*}$ and savings $s_{\tau}^{*}=f\left(k_{\tau}^{*}\right)-c_{\tau}^{*}$ are precisely the first elements of the date- $\tau$ consumer optimum starting from $k_{\tau}^{*}$ at given $\left\{r_{t+1}^{*}\right\}_{t=\tau}^{\infty}$ and $\left\{w_{t+1}^{*}\right\}_{t=\tau}^{\infty}$.

Thus the sequence $\left\{c_{t}^{*}, s_{t}^{*}, k_{t}^{*}, r_{t}^{*}, w_{t}^{*}\right\}_{t=0}^{\infty}$, given by (17), is a sliding equilibrium path under perfect foresight starting from $s_{-1}^{*}=k_{0}^{*}$, which completes the proof of Lemma 2.3 and the proof of existence theorem as well.

\section{Proof of Lemma 3.1}

Let $c_{\tau}^{* * \tau}$ be the date- $\tau$ consumption in the date- $\tau$ consumer optimum starting from $s_{\tau-1}^{*}$ at given $\left\{r_{t}^{*}\right\}_{t=\tau}^{\infty}$ and $\left\{w_{t}^{*}\right\}_{t=\tau}^{\infty}$. It follows from (6) that

$$
c_{\tau}^{* * \tau}=\frac{\left(1+r_{\tau}^{*}\right) s_{\tau-1}^{*}+w_{\tau}^{*}+\frac{w_{\tau+1}^{*}}{1+r_{\tau+1}^{*}}+\ldots+\frac{w_{\tau+t}^{*}}{\left(1+r_{\tau+1}^{*} \cdots\left(1+r_{\tau+t}^{*}\right)\right.}+\ldots}{1+(\beta \delta)^{\frac{1}{\rho}}\left(1+r_{\tau+1}^{*}\right)^{\frac{1-\rho}{\rho}}+\ldots+\left(\beta \delta^{t}\right)^{\frac{1}{\rho}}\left(1+r_{\tau+1}^{*}\right)^{\frac{1-\rho}{\rho}} \cdots\left(1+r_{\tau+t}^{*}\right)^{\frac{1-\rho}{\rho}}+\ldots} .
$$

Due to the properties of $r^{*}$ (see Lemma 1.2), the value $c_{\tau}^{* * \tau}$ is well-defined for all $\tau$. Indeed,

$$
\frac{w_{\tau+t+1}^{*}}{\left(1+r_{\tau+1}^{*}\right) \cdots\left(1+r_{\tau+t+1}^{*}\right)} \cdot \frac{\left(1+r_{\tau+1}^{*}\right) \cdots\left(1+r_{\tau+t}^{*}\right)}{w_{\tau+t}^{*}}=\frac{w_{\tau+t+1}^{*}}{w_{\tau+t}^{*}} \cdot \frac{1}{1+r_{\tau+t+1}^{*}} \underset{t \rightarrow \infty}{\longrightarrow} \frac{1}{1+r^{*}}<1
$$

and

$$
\frac{\left(\beta \delta^{t+1}\right)^{\frac{1}{\rho}}\left(1+r_{\tau+1}^{*}\right)^{\frac{1-\rho}{\rho}} \cdots\left(1+r_{\tau+t+1}^{*}\right)^{\frac{1-\rho}{\rho}}}{\left(\beta \delta^{t}\right)^{\frac{1}{\rho}}\left(1+r_{\tau+1}^{*}\right)^{\frac{1-\rho}{\rho}} \cdots\left(1+r_{\tau+t}^{*}\right)^{\frac{1-\rho}{\rho}}}=\left(\delta\left(1+r_{\tau+t+1}^{*}\right)^{1-\rho}\right)^{\frac{1}{\rho}} \underset{t \rightarrow \infty}{\longrightarrow}\left(\delta\left(1+r^{*}\right)^{1-\rho}\right)^{\frac{1}{\rho}}<1 .
$$

By the d'Alembert's ratio test both infinite series in (D.1) converge, and their sums are finite.

Let $\Delta_{t+1}$ be given by (18). By the same argument as above, $0<\Delta_{t}<\infty$ for all $t$. Now, on a SEP under perfect foresight, we have for all $\tau=0,1, \ldots$,

$$
\begin{gathered}
c_{\tau}^{*}=c_{\tau}^{* * \tau}=\frac{\left(1+r_{\tau}^{*}\right) s_{\tau-1}^{*}+w_{\tau}^{*}+\frac{w_{\tau+1}^{*}}{1+r_{\tau+1}^{*}}+\ldots+\frac{w_{\tau+t}^{*}}{\left(1+r_{\tau+1}^{*}\right) \cdots\left(1+r_{\tau+t}^{*}\right)}+\ldots}{1+(\beta \delta)^{\frac{1}{\rho}}\left(1+r_{\tau+1}^{*}\right)^{\frac{1-\rho}{\rho}}\left(1+\Delta_{\tau+1}\right)}, \\
k_{\tau+1}^{*}=s_{\tau}^{*}=\left(1+r_{\tau}^{*}\right) s_{\tau-1}^{*}+w_{\tau}^{*}-c_{\tau}^{*}, \\
1+r_{\tau}^{*}=f^{\prime}\left(k_{\tau}^{*}\right), \quad w_{\tau}^{*}=f\left(k_{\tau}^{*}\right)-f^{\prime}\left(k_{\tau}^{*}\right) k_{\tau}^{*} .
\end{gathered}
$$

Consider the value $c_{\tau+1}^{*}\left(1+\beta^{\frac{1}{\rho}} \Delta_{\tau+1}\right)$. Using (19) and (D.2), it can be checked that

$$
\begin{aligned}
& c_{\tau+1}^{*}\left(1+\beta^{\frac{1}{\rho}} \Delta_{\tau+1}\right)=c_{\tau+1}^{*}\left(1+(\beta \delta)^{\frac{1}{\rho}}\left(1+r_{\tau+2}^{*}\right)^{\frac{1-\rho}{\rho}}\left(1+\Delta_{\tau+2}\right)\right) \\
& =\left(1+r_{\tau+1}^{*}\right) s_{\tau}^{*}+w_{\tau+1}^{*}+\frac{w_{\tau+2}^{*}}{1+r_{\tau+2}^{*}}+\ldots=\left(1+r_{\tau+1}^{*}\right)\left(s_{\tau}^{*}+\frac{w_{\tau+1}^{*}}{1+r_{\tau+1}^{*}}+\frac{w_{\tau+2}^{*}}{\left(1+r_{\tau+1}^{*}\right)\left(1+r_{\tau+2}^{*}\right)}+\ldots\right) \\
& \quad=\left(1+r_{\tau+1}^{*}\right)\left(s_{\tau}^{*}+c_{\tau}^{*}\left(1+(\beta \delta)^{\frac{1}{\rho}}\left(1+r_{\tau+1}^{*}\right)^{\frac{1-\rho}{\rho}}\left(1+\Delta_{\tau+1}\right)\right)-\left(1+r_{\tau}^{*}\right) s_{\tau-1}^{*}-w_{\tau}^{*}\right) \\
& =\left(1+r_{\tau+1}^{*}\right)\left(c_{\tau}^{*}\left(1+(\beta \delta)^{\frac{1}{\rho}}\left(1+r_{\tau+1}^{*}\right)^{\frac{1-\rho}{\rho}}\left(1+\Delta_{\tau+1}\right)\right)-c_{\tau}^{*}\right)=c_{\tau}^{*}(\beta \delta)^{\frac{1}{\rho}}\left(1+r_{\tau+1}^{*}\right)^{\frac{1}{\rho}}\left(1+\Delta_{\tau+1}\right) .
\end{aligned}
$$


Therefore, on a SEP under perfect foresight the consumption levels at two adjacent dates are linked via the "first-order conditions" (20), which proves the lemma.

\section{E Proof of Theorem 4}

We prove this theorem by contradiction. Suppose that $k^{*} \leq k^{\circ}$, so that $r^{\circ} \leq r^{*}$ and $w^{\circ} \geq w^{*}$.

Let $c\left(r^{\circ}, w^{\circ}\right)$ be the date- $\tau$ consumption in the date- $\tau$ consumer optimum starting from $s^{\circ}$ at given constant over time interest rate $r^{\circ}$ and wage rate $w^{\circ}$. The following lemma shows that $k^{\circ} \geq k^{*}$ implies $c\left(r^{\circ}, w^{\circ}\right) \geq c^{\circ}$.

Lemma E.1. Suppose that $0<\rho \leq 1$, and $k^{*} \leq k^{\circ}$. Then

$$
c\left(r^{\circ}, w^{\circ}\right) \geq c^{\circ}
$$

Proof. Since by assumptions $r^{\circ} \leq r^{*}$ and $0<\rho \leq 1$, we have $\delta\left(1+r^{\circ}\right)^{1-\rho} \leq \delta\left(1+r^{*}\right)^{1-\rho}<1$. It then follows from Lemma 1.1 that $c\left(r^{\circ}, w^{\circ}\right)$ exists and is given by

$$
c\left(r^{\circ}, w^{\circ}\right)=\frac{1-\delta^{\frac{1}{\rho}}\left(1+r^{\circ}\right)^{\frac{1-\rho}{\rho}}}{1-\delta^{\frac{1}{\rho}}\left(1+r^{\circ}\right)^{\frac{1-\rho}{\rho}}+(\beta \delta)^{\frac{1}{\rho}}\left(1+r^{\circ}\right)^{\frac{1-\rho}{\rho}}} \cdot \frac{1+r^{\circ}}{r^{\circ}} \cdot\left(r^{\circ} s^{\circ}+w^{\circ}\right) .
$$

Taking into account that $c^{\circ}=\left(1+r^{\circ}\right) s^{\circ}+w^{\circ}-s^{\circ}=r^{\circ} s^{\circ}+w^{\circ}$, we get

$$
c\left(r^{\circ}, w^{\circ}\right)=Z\left(r^{\circ}\right) c^{\circ}
$$

where the function $Z(r)$ is given by

$$
Z(r)=\frac{1-\delta^{\frac{1}{\rho}}(1+r)^{\frac{1-\rho}{\rho}}}{1-\delta^{\frac{1}{\rho}}(1+r)^{\frac{1-\rho}{\rho}}+(\beta \delta)^{\frac{1}{\rho}}(1+r)^{\frac{1-\rho}{\rho}}} \cdot \frac{1+r}{r} .
$$

It follows from the proof of Lemma 1.1 that $Z\left(r^{*}\right)=1$, where $r^{*}$ is the interest rate on a SSE under perfect foresight.

Let us show that for all $r \leq r^{*}$, we have $Z(r) \geq Z\left(r^{*}\right)=1$. Repeating the argument used in the proof of Theorem 1, we get:

$$
Z(r) \geq 1 \Leftrightarrow \frac{1}{1+r} \leq \frac{\left(\frac{1}{1+r}\right)^{\frac{1}{\rho}}-(\beta \delta)^{\frac{1}{\rho}}}{\delta^{\frac{1}{\rho}}-(\beta \delta)^{\frac{1}{\rho}}}
$$

Let $\gamma=\frac{1}{1+r}$, and consider, as in the proof of Lemma 1.2, the functions $L(\gamma)=\gamma$, and $R(\gamma)=\frac{\gamma^{\frac{1}{\rho}}-(\beta \delta)^{\frac{1}{\rho}}}{\delta^{\frac{1}{\rho}}-(\beta \delta)^{\frac{1}{\rho}}}$. Since they are monotone and

$$
R\left(\gamma^{*}\right)=L\left(\gamma^{*}\right), \quad R(\delta)=1>\delta=L(\delta)
$$


it follows that $R(\gamma) \geq L(\gamma)$ for all $\gamma$ such that $\gamma^{*} \leq \gamma<\delta$. In terms of interest rates,

$$
Z(r) \geq 1 \Leftrightarrow \frac{1}{1+r} \geq \frac{1}{1+r^{*}} \Leftrightarrow r \leq r^{*}
$$

Since by assumption, $r^{\circ} \leq r^{*}$, we have $Z\left(r^{\circ}\right) \geq Z\left(r^{*}\right)=1$. Inequality (E.1) now follows from (E.2), which completes the proof of the lemma.

At the same time, the following lemma shows that the opposite inequality holds.

Lemma E.2. Suppose that $0<\rho \leq 1$, and $k^{*} \leq k^{\circ}$. Then

$$
c^{\circ}>c\left(r^{\circ}, w^{\circ}\right)
$$

Proof. Recall that $c^{\circ}$ is the date- $\tau$ consumption in the date- $\tau$ consumer optimum starting from $s^{\circ}$ at given $\left\{r_{t}^{* \tau}\right\}_{t=\tau}^{\infty}$ and $\left\{w_{t}^{* \tau}\right\}_{t=\tau}^{\infty}$. It follows from (6) that

$$
c^{\circ}=\frac{\left(1+r^{\circ}\right) s^{\circ}+w^{\circ}+\frac{w^{\circ}}{1+r^{\circ}}+\ldots+\frac{w_{\tau+t}^{* \tau}}{\left(1+r^{\circ}\right) \cdots\left(1+r_{\tau+t}^{* \tau}\right)}+\ldots}{1+(\beta \delta)^{\frac{1}{\rho}}\left(1+r^{\circ}\right)^{\frac{1-\rho}{\rho}}+\ldots+\left(\beta \delta^{t}\right)^{\frac{1}{\rho}}\left(1+r^{\circ}\right)^{\frac{1-\rho}{\rho}} \cdots\left(1+r_{\tau+t}^{* \tau}\right)^{\frac{1-\rho}{\rho}}+\ldots} .
$$

Let us check that the value $c^{\circ}$ is well-defined. Indeed, since the truncation of the date- $\tau$ optimal path which starts at date $\tau+1$ is the $\delta$-optimal path, we know that $r_{t}^{* \tau}$ and $w_{t}^{* \tau}$ monotonically converge to the corresponding modified golden rule levels for the discount factor $\delta$. Therefore,

$$
\frac{w_{\tau+t+1}^{* \tau}}{\left(1+r^{\circ}\right) \cdots\left(1+r_{\tau+t+1}^{* \tau}\right)} \frac{\left(1+r^{\circ}\right) \cdots\left(1+r_{\tau+t}^{* \tau}\right)}{w_{\tau+t}^{*}}=\frac{w_{\tau+t+1}^{* \tau}}{w_{\tau+t}^{* \tau}} \frac{1}{1+r_{\tau+t+1}^{* \tau}} \underset{t \rightarrow \infty}{\longrightarrow} \frac{1}{1+r^{\delta}}=\delta<1,
$$

and

$$
\frac{\left(\beta \delta^{t+1}\right)^{\frac{1}{\rho}}\left(1+r^{\circ}\right)^{\frac{1-\rho}{\rho}} \cdots\left(1+r_{\tau+t+1}^{* \tau}\right)^{\frac{1-\rho}{\rho}}}{\left(\beta \delta^{t}\right)^{\frac{1}{\rho}}\left(1+r^{\circ}\right)^{\frac{1-\rho}{\rho}} \cdots\left(1+r_{\tau+t}^{* \tau}\right)^{\frac{1-\rho}{\rho}}}=\left(\delta\left(1+r_{\tau+t+1}^{* \tau}\right)^{1-\rho}\right)^{\frac{1}{\rho}} \underset{t \rightarrow \infty}{\longrightarrow}\left(\delta\left(1+r^{\delta}\right)^{1-\rho}\right)^{\frac{1}{\rho}}=\delta<1,
$$

and hence by the d'Alembert's ratio test both infinite series in (E.4) converge.

Now let us compare $c^{\circ}$ and $c\left(r^{\circ}, w^{\circ}\right)$. Since $\left\{r_{t}^{* \tau}\right\}_{t=\tau+2}^{\infty}$ is the sequence of interest rates on the $\delta$-equilibrium path starting from $k^{\circ}<k^{\delta}$, it is monotonically decreasing, and $r_{t+2}^{* \tau}<r^{\circ}$ for all $t \geq \tau$. Similarly, the sequence $\left\{w_{t}^{* \tau}\right\}_{t=\tau+2}^{\infty}$ is monotonically increasing, and $w_{t+2}^{* \tau}>w^{\circ}$ for all $t \geq \tau$.

It is evident from (E.4) that $c^{\circ}$ is increasing in $w_{t}^{* \tau}$ for all $t \geq \tau+2$. Note also that the numerator in (E.4) is decreasing in $r_{t}^{* \tau}$, and for $0<\rho \leq 1$, the denominator in (E.4) is nondecreasing in $r_{t}^{* \tau}$. Hence when $0<\rho \leq 1, c^{\circ}$ is decreasing in $r_{t}^{* \tau}$ for any $t \geq \tau+2$. Therefore, inequality (E.3) holds, which proves the lemma.

Since (E.1) and (E.3) contradict each other, for $0<\rho \leq 1$ we must have $k^{*}>k^{\circ}$. It then follows that $s^{*}>s^{\circ}, r^{*}<r^{\circ}, w^{*}>w^{\circ}$, and $c^{*}=f\left(k^{*}\right)-k^{*}>f\left(k^{\circ}\right)-k^{\circ}=c^{\circ}$. 